\author{
Universidade de São Paulo \\ Instituto de Física
}

\title{
Transporte em cadeias quânticas fora do equilíbrio com potenciais quasiperiódicos
}

\author{
Artur Machado Lacerda
}

Orientador: Prof. Dr. Gabriel Teixeira Landi

Dissertação de mestrado apresentada ao Instituto de Física da Universidade de São Paulo, como requisito parcial para a obtenção do título de Mestre em Ciências.

Banca Examinadora:

Prof. Dr. Gabriel Teixeira Landi - Instituto de Física da Universidade de São Paulo

Prof. Dr. Raphael Campos Drumond - UFMG

Prof. Dr. Rodrigo Pereira - UFRN 
FICHA CATALOGRÁFICA

Preparada pelo Serviço de Biblioteca e Informação do Instituto de Física da Universidade de São Paulo

Lacerda, Artur Machado

Transporte em cadeias quânticas fora do equilíbrio com potenciais quasiperiódicos. / Transport in boundary-driven quantum chains with quasiperiodic potentials. São Paulo, 2020.

Dissertação (Mestrado) - Universidade de São Paulo. Instituto de Física. Depto. de Física dos Materiais e Mecânica

Orientador: Prof. Dr. Gabriel Teixeira Landi Área de Concentração: Física

Unitermos: 1. Fenômenos de transporte; 2. Informação quântica; 3. Mecânica quântica; 4. Mecânica estatística quântica. 


\title{
University of São Paulo
}

Physics Institute

\section{Transport in boundary-driven quantum chains with quasiperiodic potentials}

\author{
Artur Machado Lacerda
}

Supervisor: Prof. Dr. Gabriel Teixeira Landi

Dissertation submitted to the Physics Institute of the University of São Paulo in partial fulfillment of the requirements for the degree of Master of Science.

Examining Committee:

Prof. Dr. Gabriel Teixeira Landi - Instituto de Física da Universidade de São Paulo Prof. Dr. Raphael Campos Drumond - UFMG

Prof. Dr. Rodrigo Pereira - UFRN 
At this time of life, one has already been wounded many times by love; it no longer evolves solely in accordance with its own unknown and inevitable laws, before our astonished and passive heart. We come to its aid, we distort it with memory, with suggestion.

— Marcel Proust, Swann's Way 
To my parents... 


\section{Abstract}

One dimensional quasiperiodic systems have been a vibrant topic of theorical research for physicists and mathematicians alike, owing to their highly non-trivial localization properties and the fractal nature of their energy spectrum. The most paradigmatic of such systems, the Aubry-André-Harper (AAH) model, is well known for suffering a localization transition when the potential strength is increased, in which its eigenstates go from extended to localized, similarly to Anderson phase transition which occurs in disordered three-dimensional systems. The localization of the eigenstates has dramatic effects in the transport regime of the system. In this dissertation, we used numerical simulations to study the transport properties of boundary-driven fermionic quantum chains subject to two choices for the on-site potential: the aforementioned AAH model and the Fibonacci model. The transport regime is classified via the scaling of the particle current with the system size in the non-equilibrium steady state (NESS), which we calculate numerically. Our main focus was the interplay between the quasiperiodic potential and dephasing noise. We showed that even though dephasing always leads to diffusive behaviour, the transport properties of the system are still visibly affected by localization properties of the eigenstates when the coupling to dephasing is small. This influence is reflected in the scaling of the diffusion constant with the coupling. In particular, we showed that, in the localized phase of the AAH model and in the subdifusive phase of the Fibonacci model, dephasing noise may actually lead to an enhanced transport, that is, a larger value of the current compared to the zero dephasing case.

Keywords: Quasiperiodic potentials; Quantum transport; Boundary-driven chains; Open quantum systems; Anderson localization; 


\section{Resumo}

Sistemas quasiperiódicos unidimensionais tem sido um extenso objeto de pesquisa, tanto para físicos quanto para matemáticos. Esse interesse é movido, principalmente, pelas interessantes propriedades de localização desses sistemas e a natureza fractal dos espectros de energia. O mais paradigmático desses sistemas é o modelo de Aubry-André-Harper (AAH), que é bem conhecido por apresentar um transição de localização quando a força do potencial é aumentada, em que seus autoestados vão de estendidos para localizados. As propriedades de localização dos autoestados provocam consequências dramáticas no regime de transporte do sistema. Nesta dissertação, utilizamos simulações numéricas para estudar as propriedades de transporte de cadeias fermiônicas acopladas a banhos térmicos nas extremidades, sujeitas a duas escolhas de potenciais quasiperiódicos: o modelo AAH e o modelo de Fibonacci. O regime de transporte é classificado verificando-se a dependência da corrente de partícula com o tamanho do sistema no estado estacionário de não-equilíbrio (NESS), que calculamos numericamente. O trabalho focou-se principalmente na interação entre os potenciais quasiperiódicos e dephasing. Mostramos que apesar da ação do ruído de dephasing sempre induzir um comportamente difusivo, as propriedades de transporte do sistema ainda são visivelmente afetadas pelas propriedades de localização dos autoestados. Essa influência é refletida na dependência da constante de difusão com a constante de acoplamente com os banhos de dephasing. Em particular, mostramos que, na fase localizada do modelo AAH e na fase subdifusiva do modelo de Fibonacci, a ação do ruído de dephasing pode levar a um aumento do transporte, isto é, um valor de corrente maior em comparação com o caso sem dephasing.

Palavras-chave: Potenciais quasiperiódicos; Transporte quântico; Cadeias quânticas fora do equilíbrio; Sistemas quânticos abertos; Localização de Anderson; 


\section{Acknowledgements}

Agradeço ao meu orientador, Gabriel Landi, por sua excelente supervisão e pela ajuda fundamental nessa dissertação, especialmente na reta final. Iniciei meus estudos na área de Informação Quântica por acaso, com uma certa incerteza sobre o futuro. No entanto, graças à sua inspiração e após inúmeras interessantíssimas discussões, sinto-me apaixonado pela área. Não me vejo fazendo outra coisa senão Física.

Agradeço aos meus pais, Wilza e Joel, pelo amor e suporte incondicionais, essenciais para todas as conquistas da minha vida. Dedico esta dissertação a vocês. Agradeço também aos meus irmãos, Tomás e Otávio. Obrigado.

Agradeço ao meu querido amigo Leonardo, por estar sempre presente nos momentos mais difíceis. Esta dissertação também é dedicada a você.

Por fim, agradeço aos meus amigos que estiveram sempre presentes no difícil momento em que essa dissertação foi escrita. O bonde secreto: Gabriel, Tomás, Guilherme, João, JC e Tanabe. 


\section{Contents}

1 Introduction 1

2 Free fermions in a chain $\quad 7$

2.1 The tight-binding Hamiltonian $\ldots \ldots \ldots . \ldots 7$

2.2 Equivalence to the $X X$ chain $\ldots \ldots \ldots$. . . . . . . . . 9

2.3 Diagonalization . . . . . . . . . . . . . . . . 11

2.4 Constant on-site potential . . . . . . . . . . . . . . . . . 13

2.5 Anderson localization . . . . . . . . . . . . . . . 17

3 Quasiperiodic Potentials 2

3.1 Aubry-André-Harper model . . . . . . . . . . . . . . . . 22

3.2 Inverse Participation Ratio (IPR) . . . . . . . . . . . . . . 25

3.3 Fibonacci model . . . . . . . . . . . . . . . . . . . 27

4 Coherent transport $\quad 33$

4.1 Unitary time evolution . . . . . . . . . . . . . . 33

4.2 Classification of the transport regime . . . . . . . . . . . . 36

4.3 Transport properties with zero potential . . . . . . . . . . . . 38

4.4 Transport properties of the AAH model . . . . . . . . . . . . . 40

4.5 Transport properties of the Fibonacci model . . . . . . . . . . . . . . . 42

5 Open quantum systems $\quad \mathbf{4 4}$

5.1 The Lindblad master equation . . . . . . . . . . . . . . . . . . . . . . 44

5.2 Single spin-1/2 coupled to a bath . . . . . . . . . . . . . . . . . . 45

5.3 Dephasing noise . . . . . . . . . . . . . . . . . 48

5.4 Time evolution of expected values . . . . . . . . . . . . . . . . 49

5.5 Single fermionic mode . . . . . . . . . . . . . . . . . . 50

6 Boundary-driven quantum chains $\quad \mathbf{5 2}$

6.1 Boundary-driven chains . . . . . . . . . . . . . . . . . 52

6.2 Lyapunov equation . . . . . . . . . . . . . . . . . . 54

6.3 Numerical computation of the NESS . . . . . . . . . . . . . . . . . 57

6.4 Particle/spin current operator . . . . . . . . . . . . . . . . . 60

7 Non-equilibrium transport properties $\quad \mathbf{6 2}$

7.1 Classification of the transport properties via the particle current . . . . . . 62

7.2 Connection between transport coefficients . . . . . . . . . . 63 
7.3 Transport with zero potential . . . . . . . . . . . . . 65

7.4 Transport properties with quasiperiodic potentials . . . . . . . . 67

8 Dephasing enhanced transport $\quad \mathbf{7 0}$

8.1 Boundary-driven chains with dephasing . . . . . . . . . . 70

8.2 Non-equilibrium steady state . . . . . . . . . . . . . . . . . 72

8.3 Transport properties with dephasing . . . . . . . . . . . . . . 74

8.4 Quasiperiodic chains with dephasing . . . . . . . . . . . . . 78

8.5 Scaling of the conductivity . . . . . . . . . . . . . 79

9 Conclusion $\quad 86$

A The Jordan-Wigner Transformation $\quad 88$ 


\section{List of Figures}

1.1 Representation of a metal bar in the NESS. . . . . . . . . . . . . 1

1.2 A quantum chain coupled to two reservoirs at each end. . . . . . . . . . 2

1.3 Dependence of the transport coefficient $\nu$ on the potential strength in the Fibonacci model . . . . . . . . . . . . . . . . . 4

2.1 Schematic representation of the tight-biding model $\ldots \ldots . . \ldots 8$

2.2 Ground state of the tight-binding model with constant potential . . . . . . 17

2.3 Ground state in one realization of the Anderson model, with $W=0.5$. . 18

2.4 First ten excited states of the Anderson model, with $W=0.5 \ldots$. . . . . 19

2.5 Comparison of the matrix of coefficients $\left|\phi_{i}^{k}\right|^{2}$ with and without disorder . 20

3.1 Plot of the function $2 \cos (2 \pi \varphi i)$, where $\varphi=\frac{1}{2}(1+\sqrt{5}) \ldots \ldots 23$

3.2 Ground-state eigenvector of the AAH model for different values of $\lambda$. . . 24

3.3 Mean IPR as a function of $\lambda$ in the AAH model. . . . . . . . . . . . . . 26

3.4 Scaling of the IPR in the AAH model for different values of $\lambda . \ldots 27$

3.5 Schematic representation of the tight-biding model . . . . . . . . . . 28

3.6 Construction of the Fibonacci chain by cutting a sequence of the line with

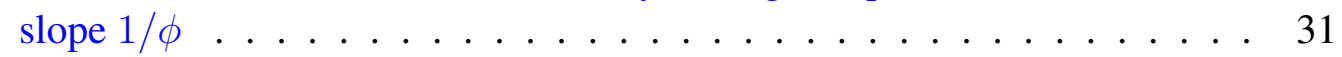

3.7 Scaling of the IPR with $L$ in the Fibonacci model . . . . . . . . . . . . 31

3.8 Dependence of the coefficient $\tau$ and of the IPR with $\lambda$ in the Fibonacci model ......................... 32

4.1 Illustration of the spreading of the wavefunction . . . . . . . . . . . . 37

4.2 Illustration of the procedure used to computed the coefficient $\alpha \ldots \ldots 39$

4.3 Scaling of the RMSD in a chain with zero potential . . . . . . . . . . 39

4.4 Scaling of the RMSD in the AAH model . . . . . . . . . . . . . . . . 41

4.5 Scaling of the root mean squared deviation of the wave function in $\mathrm{AAH}$ model. . . . . . . . . . . . . . . . . . . . 42

4.6 Dependence of the coefficient $\alpha$ with $\lambda$ in the Fibonacci model . . . . . . 43

4.7 Scaling of the RMSD in the Fibonacci model . . . . . . . . . . . . . 43

5.1 Schematic representation of a single spin coupled to a bath . . . . . . . 46

5.2 Time evolution of the populations and coherence in a single spin- $1 / 2$ coupled a bath . . . . . . . . . . . . . . . . . . 48

6.1 Schematic representation of a boundary-driven spin chain. . . . . . . . 52

7.1 Illustration of the procedure used to computed the transport coefficient $\nu$. 64 
7.2 Scaling of the current with $L$ and population profile for $L=20$ for zero on-site potential . . . . . . . . . . . . . . 67

7.3 Scaling of the current with system size in the AAH model . . . . . . . . . 68

7.4 Scaling of the current with system size in the Fibonacci model . . . . . . 69

7.5 Dependence of the exponent $\nu$ with the system size $L$ in the Fibonacci

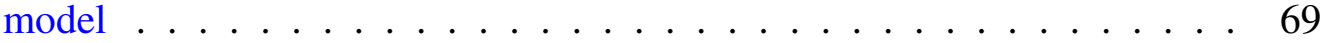

8.1 Scaling of the particle current with $L$ with zero on-site potential . . . . . 76

8.2 Scaling of the currents with system size for the AAH model with different values of $\Gamma \ldots \ldots \ldots 79 \ldots$

8.3 Scaling of the currents with system size for Fibonacci model fir different values of $\Gamma \ldots \ldots \ldots$. . . . . . . . . . . . . 80

8.4 Scaling of the conductivity with $\Gamma$ in the Fibonacci model . . . . . . . . . 84

8.5 Coefficient $\beta$ fitted from the relation $\kappa \sim \Gamma^{\beta} \ldots \ldots \ldots$. . . . . . . . 84

8.6 Scaling of the conductivity with $\Gamma$ in the AAH model $\ldots \ldots . . . . .85$ 


\section{List of Tables}

4.1 Classification of the coherent transport regime using the value of $\alpha . \ldots 38$

7.1 Classification of the non-equilibrium transport regime using the value of $\nu .63$ 


\section{Chapter 1}

\section{Introduction}

When a metal bar is put in contact with two baths at different temperatures, one at each end, a heat current $J$ will start to flow, dictated by Fourier's law,

$$
J=-\kappa \nabla T
$$

where $\nabla T$ is the temperature gradient and $\kappa$ is the thermal conductivity. Eventually, the systems settles in a steady-state, in which the temperature profile stops changing. The system, however, is not in equilibrium: each point has a different temperature and there is still a non-zero heat current, as ilustrated in Fig. 1.1. For this reason, the final state of the system is called a non-equilibrium steady state (NESS).

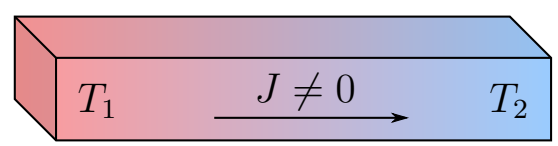

Figure 1.1: Representation of a metal bar in the NESS.

For the simple case where the system is isotropic in the $y$ and $z$ direction, the final temperature profile will be a linear interpolation between $T_{1}$ and $T_{2}$, so the heat current in the NESS will be given by

$$
J=\frac{\kappa\left(T_{2}-T_{1}\right)}{L} \sim \frac{1}{L}
$$

where $L$ is the size of the bar. Therefore, the current scales inversely proportional with the system size. This type of scaling is known as diffusive scaling. 
Surprisingly, despite the simplicity of this example, taught even in elementary physics courses, it hides a challenging open problem in theoretical physics [1, 2]. Fourier's law is a phenomenological equation. It turns out that deriving it from microscopic principles is an extremely difficult task, and no satisfactory derivation exist to this date. The first candidate that comes to mind is a chain of harmonic oscillators coupled to heat baths at its boundaries. However, it is known that this model fails to exhibit a diffusive behavior. In the famous 1967 paper by Rieder, Lebowitz and Lieb [3], they showed that when a classical harmonic lattice is coupled to baths at its boundaries at temperatures $T_{1}$ and $T_{L}$, the heat current in NESS is proportional to the temperature difference $T_{L}-T_{1}$, rather than to the gradient $\left(T_{L}-T_{1}\right) / L$. Therefore, the current in the NESS is independent of the system size:

$$
J \sim L^{0}
$$

which charaterizes a ballistic scaling. Moreover, much to their surprise, they found that the temperature profile away from the boundaries is almost constant, instead of being a linear interpolation from $T_{1}$ to $T_{L}$. Their discovery has sparkled the interest in heat transport through harmonic chains, both quantum and classical, which remains an active topic of research ever since.

In this dissertation, we will focus on the quantum version of this problem. We will consider one-dimensional quantum chains, of spins or fermions, coupled to baths at each end, the so called boundary-driven chains, as illustrated in Fig. 1.2.

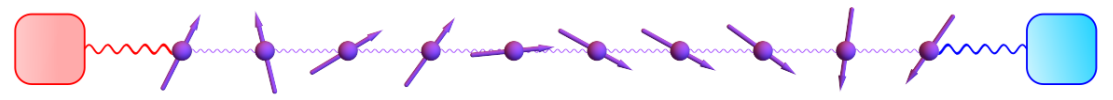

Figure 1.2: A quantum chain coupled to two reservoirs at each end.

In the quantum realm, non-iteracting chains, either bosonic of fermionic, also have a natural tendency to be ballistic [4-6], at least without any extra ingredients [7, 8]. This is, however, not always the case. The most notable counter-example is that of a chain subject to disorder, which consists in a random potential. In the seminal paper by Anderson [8, 9], he showed that when a tight-binding chain is subject to a sufficiently strong disorder, the transport is completely halted. Therefore, if a disordered chain is coupled to heat baths 
at its boundaries, the current in the NESS will be identically zero in the thermodynamic limit.

The absence of transport with disorder is closely associated with the localization properties of the eigenstates of the system. Without any on-site potential, the energy eigenstates are simple plane waves, which are extended through all the sites of the chain. In the presence of disorder, however, all the energy eigenstates become exponentially localized, which effectively cause the system to behave as an insulator. This phenomemon is known as Anderson localization, and lies at the core of condensed-matter physics. Later, it was shown that for one and two-dimensional systems the localization always happens, even for arbitrarily small disorder. In three dimensions, however, the situation is different. For weak disorder, extended eigenvectors can exist, but they all become localized when the disorder strength is increased. Therefore, for some critical value of disorder strength the system undergoes a localization transition, going from extended to localized. This is the so called metal-insultator transition (MIT).

Despite this counter-example, the notion that non-interacting chains are ballistic is still widespread. The topic of this dissertation truly contradicts this belief. The main models we studied are boundary-driven chains subject to a very interesting class of potentials called quasiperiodic. The fractal nature of the spectrum of these models has dramatic consequences on their localization properties. The quasiperiodic potentials are generated by completely deterministic functions, as opposed to the disordered potentials. Surprisingly, however, they are able to exhibit different types of transport regime even in one-dimension.

The main framework we will use to address these different transport properties is by analyzing the scaling of the particle current with the system size in NESS. In general, this scale follows

$$
J \sim \frac{1}{L^{\nu}}
$$

where $\nu$ is constant called transport coefficient. The transport regime is then classified depending on the value of $\nu$.

In this dissertation, we considered two of the most paradigmatic quasiperiodic sys- 
tems: the Aubry-André-Harper (AAH) $[10,11]$ and the Fibonacci model [12-15]. The AAH model is known to undergo a critical localization transition when the potential strength is increased, even in a one-dimensional chain. Below the critical value, all the energy eigenstates are delocalized, while above this value they all become exponentially localized. This transition is clearly reflected in the non-equilibrium transport properties of the system: at the localized region, the transport is ballistic, while above the critical value the current decays exponentially with the system size. At the critical point, the eigenstates are neither delocalized nor localized, but some intermediate between the two, and the transport is either diffusive or very close to diffusive, depending on some other parameters $[16,17]$.

The Fibonacci model displays even more interesting transport properties. The potential of this model is constructed from a peculiar binary sequence known as Fibonacci word, firstly introduced by Leonardo Fibonacci in his studies of the population dynamics of rabbits. The key feature of this model is that it can be tuned to any transport regime, depending on the value of the potential strength. When the potential is increased, the transport regime goes continuously from ballistic to subdiffusive, and in the infinite limit the eigenstates become localized. This behavior is illustrated in Fig. 1.3. As shown in the plot, the transport coefficient $\nu$ increases smoothly with the potential strength.

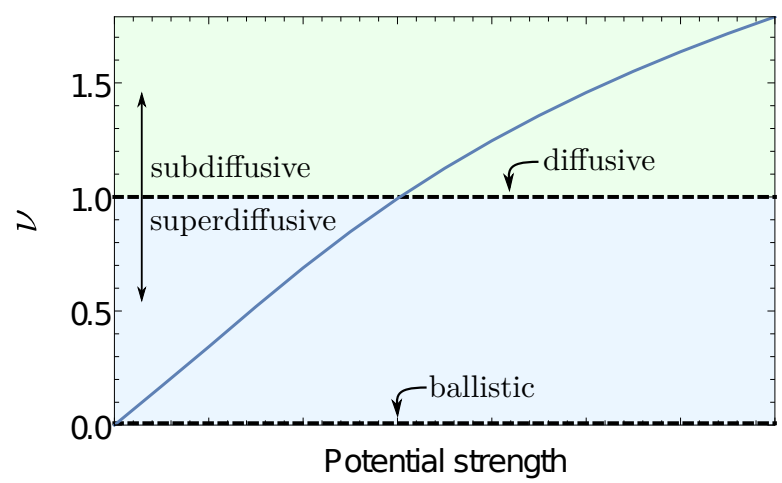

Figure 1.3: Dependence of the transport coefficient $\nu$ with the potential strength in the Fibonacci model. This curve was computed numerically, as will be explained in Chapter 7.

These unusual properties of the AAH and the Fibonacci model have been known since the 1980s [15]. However, and surprisingly, these results are not widespread known by the quantum transport community, and the notion that non-interacting chains should be bal- 
listic remains present. One of the factors that reignited the interest in these models is their potential realization in ultracold atoms experiments [18-21]. Experimental realizations of the Anderson model have also been reported [22]. These experiments have been made possible due to the major experimental advances in optical lattices and atom trapping.

When interactions are taken into account, it is indeed possible to obtain a diffusive regime. However, they often render the problem intractable. For this reason, as we briefly mentioned, there exist some extra "ingredients" that can be used to enforce diffusion in non-interacting chains, classical or quantum. One of the first successful attempts is the use of self-consistent baths [23], also known as Büttiker probes [24]. In this approach, each site is coupled to an extra bath, which is specially constructed such that it does not exchange heat or energy with the system. The sole purpose of these baths is to emulate non-harmonic interactions whilst being mathematically treatable. The self-consistent baths correctly lead to diffusion, but they are rather artificial tools, lacking any precise physical interpretation.

A second, more phenomenological approach, is the use of dephasing noise. The presence of dephasing always renders the transport diffusive, for any non-zero coupling. Nonetheless, in the weak coupling regime there is still some detectable influence of the Hamiltonian on the transport regime. In Ref. [25], the authors studied the interplay between disorder and dephasing in a $X X Z$ chain. As was discussed there, the disordered $X X Z$ has a subdiffusive phase for a certain choice of parameters. Surprisingly, by analyzing the scaling of the diffusion constant, which is the analogous to the conductivity in Fourier's law, they showed that the presence of dephasing can actually increase the current in this subdiffusive phase. They refer to this phenomenon as dephasing-enhanced transport.

Both models we studied also have subdiffusive phases. The AAH model is diffusive in the critical point, and so is the Fibonacci model when the potential strength is strong. Therefore, it is only natural to apply the same framework developed in Ref. [25] to study the interplay between dephasing and the quasiperiodic potential strength. Similarly, we have also found that dephasing can actually increase the value of the current in the subdiffusive phase of both models. This is the main result of this dissertation, and is being prepared for a publication.

This dissertation is structured as follows: 
- In chapter 2, we present the main Hamiltonian used throughout this work, and discuss its diagonalization procedure. We also discuss the phenomenon of Anderson localization.

- In chapter 3, we present the AAH and the Fibonacci models in detail, and discuss their ground state localization properties.

- In chapter 4 , we study the transport properties of both models in a closed system scenario, by measuring the spreading of the wavefunction in a unitary dynamics.

- In chapter 5, we give a brief introduction to open quantum systems and provide some insightful examples.

- In chapter 6 , we then present the main model of this work, the boundary-driven chains. We will show that in the NESS the covariance matrix of the fermionic operators satisfies a Lyapunov Equation, and discuss its numerical solutions. We also present the Mathematica code we used in our simulations.

- In chapter 7, we study the non-equilibrium transport of boundary-driven chains, by measuring the scaling of the particle current with the system size.

- Finally, in chapter 8 , we present the main original contributions of this work, the interplay between dephasing and the quasiperiodic potentials. We show that dephasing can actually lead to an increased transport in the subdiffusive phase.

All the numerical calculations performed in this dissertation were made using efficient functions written in Mathematica. The code is still being adjusted, and will be made freely available when finished. Throughout the text, we also provide explicit snippets for functions that we have developed and which, we believe, may be of use to other researchers studying similar topics. 


\section{Chapter 2}

\section{Free fermions in a chain}

In the first section of this chapter, we will present the one-dimensional fermionic tightbinding Hamiltonian. Then, in section 2.2 we will show how it is related to the $X X$ spin chain via the Jordan-Wigner transformation [26]. We will then describe the diagonalization procedure and provide an example. In the last section, we will discuss Anderson localization phenomenon. The discussion is then concluded in chapter 3 , where we consider quasi-periodic models, which will be the main focus of this dissertation.

\subsection{The tight-binding Hamiltonian}

We consider a chain with $L$ fermionic modes with open boundary conditions, subject to a site-dependent potential. In the language of second quantization, the Hamiltonian is given by

$$
\mathcal{H}=-J \sum_{i=1}^{L-1}\left(c_{i}^{\dagger} c_{i+1}+c_{i+1}^{\dagger} c_{i}\right)+\sum_{i=1}^{L} V_{i} c_{i}^{\dagger} c_{i}
$$

where $c_{i}^{\dagger}$ and $c_{i}$ are the fermionic creation and annihilation operators for site $i=1, \ldots, L$ which follow the standard fermionic anticommutation rules;

$$
\left\{c_{i}, c_{j}^{\dagger}\right\}=\delta_{i j} \quad \text { and } \quad\left\{c_{i}, c_{j}\right\}=\left\{c_{i}^{\dagger}, c_{j}^{\dagger}\right\}=0
$$

The parameter $J$ is the hopping term and $V_{i}$ is the on-site potential for site $i$. Throughout 
this work, we will choose $J=1$, fixing the energy scale. In this dissertation we will explore the broad variety of effects that emerge from different choices for the on-site potentials $V_{i}$.

The Hamiltonian in Eq. (2.1) is paradigmatic in condensed-matter physics, where it is used as an approximate model to describe the dynamics of electrons in a lattice of atoms. In the so called tight-binding approximation [27], each electron is typically bound to an atom's core, thus its position can only assume the discrete values corresponding to the lattice sites. In the case of (2.1), these positions would correspond to the sites in a one-dimensional chain. However, there is always a non-zero probability that the electron tunnels to a neighboring site. In the language of second quantization, this hopping movement corresponds to the term $c_{i+1}^{\dagger} c_{i}$, which creates a particle in site $i+1$ and destroys a particle in site $i$ with amplitude $J$. The hermitian conjugate $c_{i}^{\dagger} c_{i+1}$ is responsible for the hopping in the opposite direction. Fig. 2.1 depicts a schematic representation of this model.

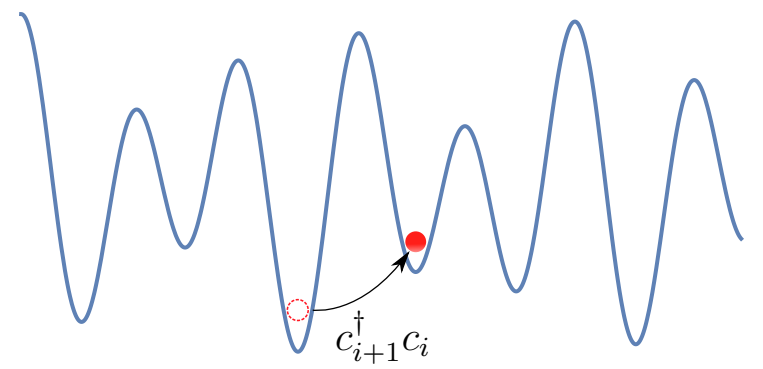

Figure 2.1: Schematic representation of the tight-biding model, Eq. (2.1). The model is described in a one-dimensional lattice, with L sites. Each site has a different on-site energy and particles can hop from one site to another with a tunneling rate $J$. The term $c_{i+i}^{\dagger} c_{i}$ creates a particle in site $i+1$ and destroys a particle in site $i$.

Notice that the Hamiltonian (2.1) preserves the total number of particles. This can be seen by defining the total number operator,

$$
\mathcal{N}=\sum_{i=1}^{L} c_{i}^{\dagger} c_{i}
$$

and verifying that it commutes with $\mathcal{H}$,

$$
[\mathcal{H}, \mathcal{N}]=0 .
$$


Intuitively, each term of the form $c_{i}^{\dagger} c_{j}$ commutes with $\mathcal{N}$ because it destroys a particle in site $j$ and creates a particle in site $i$, thus preserving the total number of particles. Since $\mathcal{N}$ commutes with the Hamiltonian, the expected value $\langle\mathcal{N}\rangle$ is constant during a unitary time evolution. For this reason, if the initial state has a single particle, it will continue to have so during the whole evolution.

\subsection{Equivalence to the $X X$ chain}

The tight-binding model (2.1) turns out to be mathematically equivalent to a chain of spin $1 / 2$ particles. This provides a dual interpretation to the model. In this section we describe how to construct this mapping. For the remainder of the dissertation, we will focus on the fermionic representation for convenience. However, we stress here that, through this mapping, all results can also be reinterpreted in the language of spin chains.

The Hamiltonian for a $X X$ spin chain is given by

$$
\mathcal{H}=-2 J \sum_{i=1}^{L-1}\left(\sigma_{i}^{x} \sigma_{i+1}^{x}+\sigma_{i}^{y} \sigma_{i+1}^{y}\right)+\frac{1}{2} \sum_{i}^{L} V_{i} \sigma_{i}^{z}
$$

where $V_{i}$ is the magnetic field and and the operators $\sigma_{i}^{x, y, z}$ are the Pauli matrices,

$$
\sigma_{i}^{x}=\left(\begin{array}{cc}
0 & 1 \\
1 & 0
\end{array}\right), \quad \sigma_{i}^{y}=\left(\begin{array}{cc}
0 & -i \\
i & 0
\end{array}\right) \quad \text { and } \quad \sigma_{i}^{z}=\left(\begin{array}{cc}
1 & 0 \\
0 & -1
\end{array}\right)
$$

This system is called $X X$ model because it is isotropic, that is, the coupling terms in directions $X$ and $Y$ have the same coefficient. By defining the Pauli rasing and lowering operators $\sigma_{i}^{ \pm}=\frac{1}{2}\left(\sigma_{i}^{x} \pm i \sigma_{i}^{y}\right)$, Eq. 2.5 can be written in the more convenient form

$$
\mathcal{H}=-J \sum_{i=1}^{L-1}\left(\sigma_{i}^{+} \sigma_{i+1}^{-}+\sigma_{i}^{-} \sigma_{i+1}^{+}\right)+\frac{1}{2} \sum_{i}^{L} V_{i} \sigma_{i}^{z}
$$

This Hamiltonian can be written in terms of fermionic operators using the JordanWigner transformation, defined as

$$
c_{i}=\left[\bigotimes_{k=1}^{i-1}\left(-\sigma_{k}^{z}\right)\right] \sigma_{i}^{-}
$$


Under this transformation, the XX spin chain is mapped into the tight-binding Hamiltonian of Eq. (2.1). Additional details about the transformation (2.8) are given in Appendix A. We also remark that, provided one considers only open-boundary conditions, none of the usual difficulties concerning the parity of the operators arise.

It is important to remark that when the fermionic tight-binding model is obtained via a Jordan-Wigner transformation, the underlying physical system is still a spin chain. Therefore, the fermionic excitations do not correspond to actual particles, but to excitations in the spin chain. In fact, the transformation naturally maps fermionic excitations into states with spin up, that is

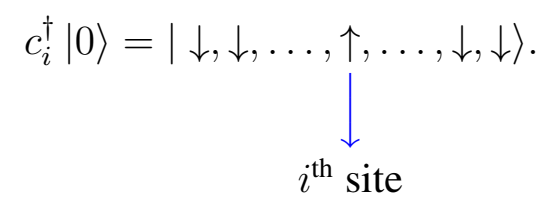

The vacuum state $|0\rangle$, in particular, corresponds to a state with all the spins down. With this correspondence in mind, both models are completely equivalent. Throughout this dissertation, we will remain exclusively in the fermionic formulation, but the all the results hold for the $X X$ spin chain as well.

As a consequence of (2.8), the total number of fermionic particles can be related to the average magnetization in the spin chain, the magnetization is defined as

$$
m=\frac{1}{L} \sum_{i=1}^{L}\left\langle\sigma_{i}^{z}\right\rangle .
$$

From (2.8), the operator $\sigma_{i}^{z}$ is related to the fermionic operators according to

$$
\sigma_{i}^{z}=2 c_{i}^{\dagger} c_{i}-1
$$

Using this mapping in (2.10) results in

$$
m=\frac{2\langle\mathcal{N}\rangle}{L}-1
$$

Therefore, the magnezation in the spin chain is also preserved during any unitary time evolution. 


\subsection{Diagonalization}

Generic quadratic Hamiltonians of the form (2.1) can always be diagonalized exactly. This is one of the major advantages of free fermion models. The Hamiltonian can be written in the more general form

$$
\mathcal{H}=\sum_{i j} H_{i j} c_{i}^{\dagger} c_{j}
$$

where $H$ is a hermitian tridiagonal $L \times L$ matrix given by

$$
H=\left(\begin{array}{ccccc}
V_{1} & -1 & & & \\
-1 & V_{2} & -1 & & \\
& -1 & \ddots & \ddots & \\
& & \ddots & \ddots & -1 \\
& & & -1 & V_{L}
\end{array}\right)
$$

As we now discuss, the full Hamiltonian $\mathcal{H}$ can be diagonalized by simply diagonalizing the $L \times L$ matrix $H$. The eigendecompositon of $H$ can be written as

$$
H=S \mathcal{E} S^{\dagger}
$$

where $\mathcal{E}=\operatorname{diag}\left(\epsilon_{1}, \ldots, \epsilon_{L}\right)$ is a diagonal matrix containing the eigenvalues and and $S$ is a unitary matrix $\left(S S^{\dagger}=\mathbb{1}\right)$ whose columns are the corresponding eigenvectors. This equation can be written elementwise as

$$
H_{i j}=\sum_{k} S_{i k} \epsilon_{k} S_{j k}^{*}
$$

By inserting this expression in Eq. (2.13), we obtain

$$
\begin{aligned}
\mathcal{H} & =\sum_{i j} H_{i j} c_{i}^{\dagger} c_{j} \\
& =\sum_{i j} \sum_{k} S_{i j} \epsilon_{k} S_{j k}^{*} c_{i}^{\dagger} c_{j} \\
& =\sum_{k} \epsilon_{k}\left(\sum_{i} S_{i k} c_{i}^{\dagger}\right)\left(\sum_{j} S_{j k}^{*} c_{j}\right) .
\end{aligned}
$$


This motivates the definition of a new set of operators,

$$
b_{k}^{\dagger}=\sum_{i} S_{i k} c_{i}^{\dagger} \quad \text { and } \quad b_{k}=\sum_{j} S_{j k}^{*} c_{j}
$$

which we shall refer to as "energy modes". One may verify that, since $S$ is unitary, the $b_{k}$ also satisfy (2.2) and thus represent a valid set of fermionic operators. In terms of these new operators the Hamiltonian is diagonal,

$$
\mathcal{H}=\sum_{k} \epsilon_{k} b_{k}^{\dagger} b_{k}
$$

The eigenvalues of the full second-quantized Hamiltonian $\mathcal{H}$ can now be deduced from the the fact that each $b_{k}$ represents an independent fermionic operator, so that $b_{k}^{\dagger} b_{k}$ can have eigenvalues $n_{k}=0,1$. The set of quantum numbers characterizing the eigenvalues of $\mathcal{H}$ is thus given by $\left\{n_{k}\right\}$, the set of all occupation numbers of the $b_{k}$ operators. The corresponding eigenvalues are

$$
E_{n_{1}, \ldots, n_{L}}=\sum_{k} \epsilon_{k} n_{k},, \quad n_{k}=0,1
$$

where $n_{k}=\left\langle b_{k}^{\dagger} b_{k}\right\rangle$ is the fermionic occupation number of energy mode $k$. The corresponding eigenstates can be written as

$$
\left|E_{n_{1}, \ldots, n_{L}}\right\rangle=\prod_{k=1}^{L}\left(b_{k}^{\dagger}\right)^{n_{k}}|0\rangle .
$$

If the total number of fermions is not fixed, the Hamiltonian (2.1) acts on the entire Fock space, which is $2^{L}$-dimensional. This means that $\mathcal{H}$ has $2^{L}$ eingenvalue/eigenstate pairs, corresponding to all the possible combinations of $n_{1}, \ldots, n_{L}$. Throughout this dissertation, however, we shall always consider the single-particle case, that is, when there is only a single particle hopping through the lattice.

When this restriction to single particle states is made, the natural basis is the set $\{|1\rangle, \ldots,|L\rangle\}$, where $|i\rangle$ is the state in which the particle is in site $i$, that is,

$$
|i\rangle=c_{i}^{\dagger}|0\rangle
$$


Any single-particle state can thus be expanded as

$$
|\psi\rangle=\sum_{i=1}^{L} \psi_{i}|i\rangle
$$

where the coefficients $\psi_{i}$ constitute the wave function in position space.

Furthermore, since in this case at most one $n_{k}$ is different from zero in Eq. (2.20), the eigenvalues of the system are simply $\epsilon_{1}, \ldots, \epsilon_{L}$, the same as those of the $L \times L$ matrix $H$. The corresponding eigenstates are

$$
\left|\phi_{k}\right\rangle=b_{k}^{\dagger}|0\rangle
$$

which can be written as an expansion in the basis $\{|i\rangle\}$ using the transformations in Eq. (2.18), resulting in

$$
\left|\phi_{k}\right\rangle=\sum_{i=1}^{L} S_{i k}|i\rangle
$$

From this equation, it is possible to see that the eigenstates of the system match the eigenvectors of $H$, given by the columns of $S$. In fact, the single-particle Hamiltonian is often writen as

$$
H=-\sum_{i=1}^{L}(|i\rangle\langle i+1|+| i+1\rangle\langle i|)+\sum_{i=1}^{L} V_{i}|i\rangle\langle i| .
$$

Within the context of free fermions, the single-particle and the second quantized Hamiltonians are thus in one-to-one correspondence.

\subsection{Constant on-site potential}

As and illustratrive example, we shall first consider the tight-binding model with constant potential $V$, that is,

$$
V_{i}=V, \quad i=1, \ldots, L
$$


For this model exclusively, we will consider both open and periodic boundary conditions (PBC). This is also the only model we will be able to solve analytically. For the other potentials, numerical diagonalization procedures will be used.

\section{Periodic boundary conditions}

When periodic boundary conditions are considered, the single-particle Hamiltonian $H$ has the form

$$
H=\left(\begin{array}{rrrrr}
V & -1 & & & -1 \\
-1 & V & -1 & & \\
& -1 & \ddots & \ddots & \\
& & \ddots & \ddots & -1 \\
-1 & & & -1 & V
\end{array}\right)
$$

where the only difference with respect to (2.14) is the presence of the two "-1"'s at the corners. The eigenvalue equation $H|\phi\rangle=\epsilon|\phi\rangle$ can now be written as a recursion relation for each coefficient $\phi_{n}$ of the eigenvectors:

$$
-\left(\phi_{n-1}+\phi_{n+1}\right)+V \phi_{n}=\epsilon \phi_{n}, \quad n=1, \ldots, L,
$$

with $\psi_{L+1}=\psi_{1}$. This can be solved by introducing the plane wave ansatz,

$$
\phi_{n}^{k}=\frac{1}{\sqrt{L}} e^{i k n}
$$

where $k$ are quantum numbers to be determined. Periodic boundary conditions imply that $e^{i k L}=1$. Hence, the allowed values of $k$ are

$$
k=\frac{2 \pi l}{L}, \quad l \in \mathbb{Z}
$$

Each eigenvalue/eigenvector pair is labeled by one value of $k$. Since $H$ is $L \times L$, one requires only $L$ distinct values of $k$. There is, therefore, some arbitrariness in the choice of values of $k$. Usually, $k$ is taken inside the interval $[-\pi, \pi]$, known as the first Brillouin zone. Thus, the allowed values for $l$ are 


$$
-\frac{L}{2}<l \leq \frac{L}{2}
$$

The eigenvalues can be obtained by plugging the ansatz in Eq. (2.29), which results in

$$
-\frac{1}{\sqrt{L}} e^{i k n}\left(e^{i k}+e^{-i k}-V\right)=\frac{\epsilon_{k}}{\sqrt{L}} e^{i k n}
$$

This shows that (2.30) can indeed be a valid eigenstate of $H$, provided that the corresponding eigenvalue reads

$$
\epsilon_{k}=V-2 \cos k
$$

which is the well-known dispersion relation of the tight-binding model. The corresponding eigenstates are

$$
\left|\phi_{k}\right\rangle=\frac{1}{\sqrt{L}} \sum_{n=1}^{L} e^{i k n}|n\rangle
$$

The ground state, in particular, corresponds to $k=0$, and is given by

$$
\left|\phi_{\mathrm{gs}}\right\rangle=\frac{1}{\sqrt{L}} \sum_{n=1}^{L}|n\rangle .
$$

Notice that all eigenstates are completely delocalized, that is, the wave function is uniformly distributed along the chain,

$$
\left|\phi_{n}^{k}\right|^{2}=\frac{1}{L}, \quad n=1, \ldots, L
$$

\section{Open boundary conditions}

For open boundary conditions, Eq. (2.29) continues to hold, provided that we impose $\psi_{0}=\psi_{L+1}=0$. The ansatz (2.30) still solves this equation, with the eigenvalues also given by $\epsilon_{k}=V-2 \cos k$ [Eq. (2.34).] However, it is incompatible with the open boundary conditions. This can be amended by introducing the new ansatz 


$$
\phi_{n}^{k}=A e^{i k n}+B e^{-i k n}
$$

where $A$ and $B$ are constants. By inserting this ansatz in Eq. (2.29), we obtain

$$
-\left(A e^{i k n}+B e^{-i k n}\right)\left(e^{i k}+e^{-i k}-V\right)=\epsilon_{k}\left(A e^{i k n}+B e^{-i k n}\right) .
$$

This shows that (2.38) also solves Eq. (2.29) with the same dispersion relation as in Eq. (2.34). The condition $\phi_{0}=0$ now imposes that $B=-A$. Thus,

$$
\phi_{n}^{k}=2 i A \sin (k n)
$$

Finally, setting $\psi_{L+1}=0$ then implies that $\sin (k(L+1))=0$, or

$$
k=\frac{\pi l}{L+1}, \quad l=1, \ldots, L
$$

Note that the allowed values of $k$ are slightly different in comparison with (2.32). The constant $A$ is obtained by imposing the normalization of the wave function:

$$
\sum_{n=1}^{L}\left|\phi_{n}^{k}\right|^{2}=4|A|^{2} \sum_{n=1}^{L} \sin (k n)=1
$$

which yields

$$
|A|=\sqrt{\frac{1}{2(L+1)}} .
$$

The complex phase of the normalization constant is arbitrary, thus the final form of the eigenstates can be written as

$$
\left|\phi_{k}\right\rangle=\sqrt{\frac{2}{L+1}} \sum_{n=1}^{L} \sin (k n)|n\rangle,
$$

where the allowed values of $k$ are determined by Eq. (2.41). The ground state, in particular, corresponds to $l=1$.

Fig. 2.2 shows the ground state for a lattice with $L=100$. Notice how the state is 
delocalized, with the wave function spread throughout all the sites. This will be contrasted in the next section, where we will discuss the Anderson localization phenomenon. We notice that this model is the discrete analog of a particle in an infinite square well, and the eigenstates obtained in the continuous case are very similar to (2.44), but with a different normalization factor.

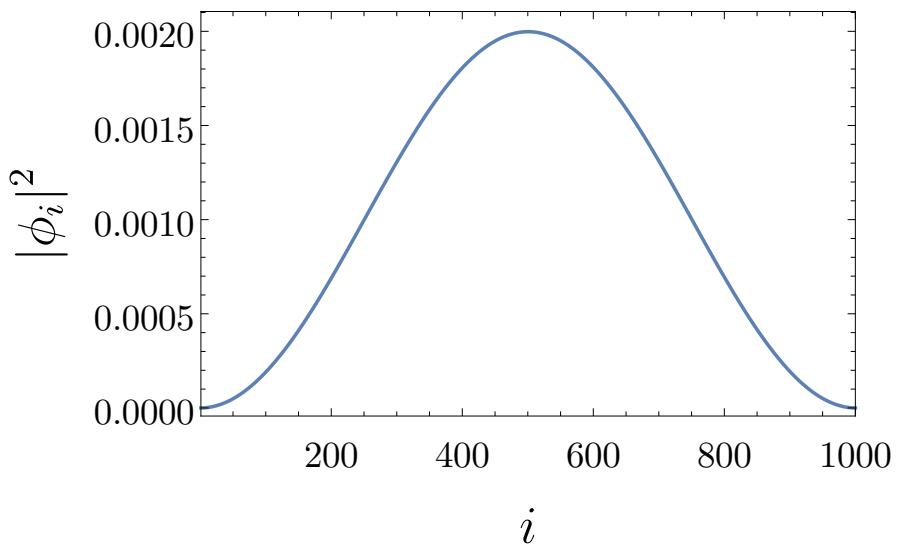

Figure 2.2: Ground state of the tight-binding model with constant potential for $L=$ 1000. The $y$ axis shows the squared modulus of the coefficients in the expansion $\left|\phi_{\mathrm{gs}}\right\rangle=$ $\sum_{i} \phi_{i}|i\rangle$.

\subsection{Anderson localization}

In the seminal work published by P.W. Anderson [9] in 1958, he showed that when a tightbinding lattice is subject to a strong enough disorder, that is, a random on-site potential $V_{i}$, all the eigenstates of the system become exponentially localized. As a consequence, the wave function ceases to propagate in a unitary dynamics, thus effectively turning the system into an insulator. This phenomenon is known as Anderson localization. Anderson's work is widely regarded as a corner-stone in condensed-matter physics and is one of the reasons he was awarded a Nobel prize in 1977.

In his original work, Anderson was motivated by the study of nuclear spin diffusion and conduction of electrons. In the following decades, however, the concept of Anderson localization has been applied into a wide variety of fields, even outside solid state Physics, such as photonics [28], mesoscopic Physics [29], acoustic waves [30] and electron transport in DNA molecules [31]. The localization phenomenon has also been a topic 
of intense experimental research. It has, for example, been observed in ultracold atoms experiments [22].

In the last decade, significant breakthroughs have been made in the field of ultracold atoms, thus creating exciting possibilities for experimental simulation of many-body systems. These advances have led to a revival of the interest in disordered systems and localization properties. A particular active topic of research is the study of many-body localization (MBL), a different localization phenomenon which occurs in interacting manybody systems $[32,33]$.

We consider here the one-dimensional version of the Anderson the model. The Hamiltonian of the system is given by Eq. (2.1), and the on-site potentials $V_{i}$ are chosen as independent and identically distributed random variables sampled from a uniform distribution in the range $\left[-\frac{W}{2}, \frac{W}{2}\right]$. The parameter $W$, which is the width of the distribution, controls the strength of the disorder.

The eigenstates of the model can be found numerically using the exact diagonalization procedure described in section 2.3. Fig. 2.3 shows the ground state for one realization of the model for $W=0.5$ in a lattice with 1000 sites. Notice that almost all the probability in concentrated near one site, which is called localization center, as opposed to the zero disorder case shown in Fig. 2.2. The particular site in which the wave function is localized is random, depending on the particular realization of the disordered potential.

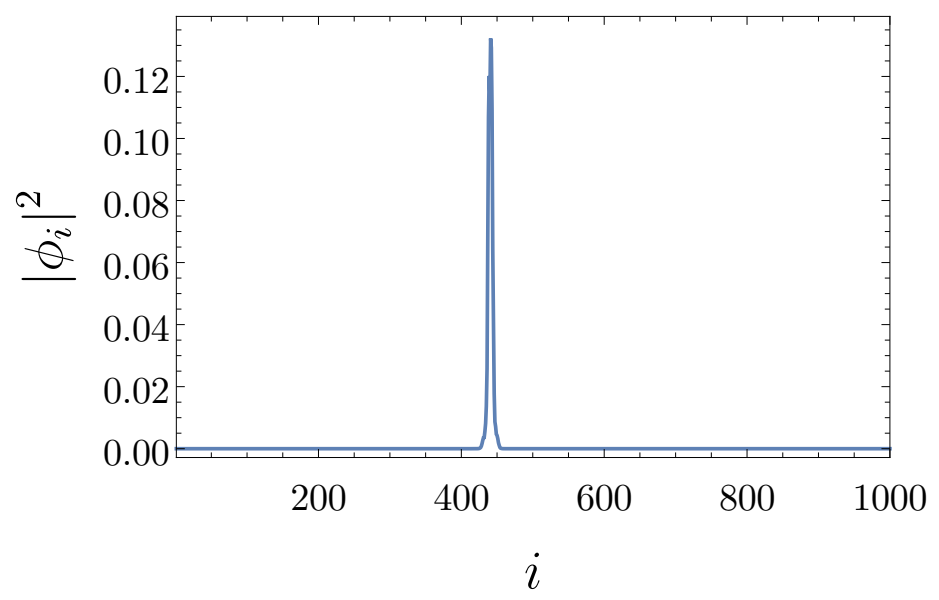

Figure 2.3: Ground state in one realization of the Anderson model, with $W=0.5$ and $L=1000$.

Not only is the ground state localized, but also all the remaining eigenstates. Fig. 2.4 
shows the first 10 eigenstates of the same realization of the disorder used in Fig. 2.3, with disorder $W=0.5$. Notice that each eigenstate is localized in some particular site.

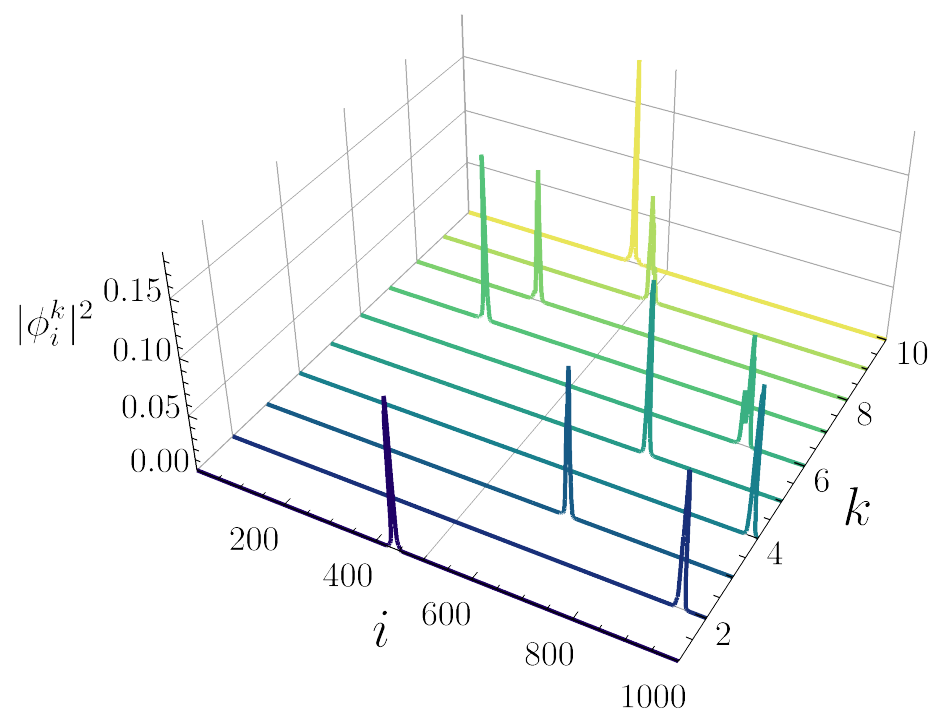

Figure 2.4: First ten excited states of the Anderson model, with $W=0.5$ and $L=1000$. The index $k$ labels the different eigenvalues.

The localization for all eigenstates can be visualized simultaneously by plotting the matrix of coefficients $\left|\phi_{i}^{k}\right|^{2}$, where $\phi_{i}^{k}$ are the expansion coefficients of the $k^{\text {th }}$ eigenstate,

$$
\left|\phi_{k}\right\rangle=\sum_{i=1}^{L} \phi_{i}^{k}|i\rangle .
$$

This corresponds to the matrix $S$, as defined in Eq. (2.15), but with all elements squared. Fig. 2.5 shows a comparison between zero disorder and $W=0.5$ for a lattice with $L=$ 100. Each column of the matrix corresponds to an eigenvector, and each row corresponds to a site in the lattice.

In Anderson's original paper, it is shown that if the disorder is sufficiently strong, all eigenstates become localized, regardless of the dimension of the system. It was later found that for one and two-dimensional systems the localization occurs for any disorder, no matter how small $[34,35]$. We give here a brief sketch of the proof. The recursive relation (2.29) can be written in matrix notation as

$$
\left(\begin{array}{c}
\phi_{n+1} \\
\phi_{n}
\end{array}\right)=T_{n}\left(\begin{array}{c}
\phi_{n} \\
\phi_{n-1}
\end{array}\right), \quad 2<n<L
$$




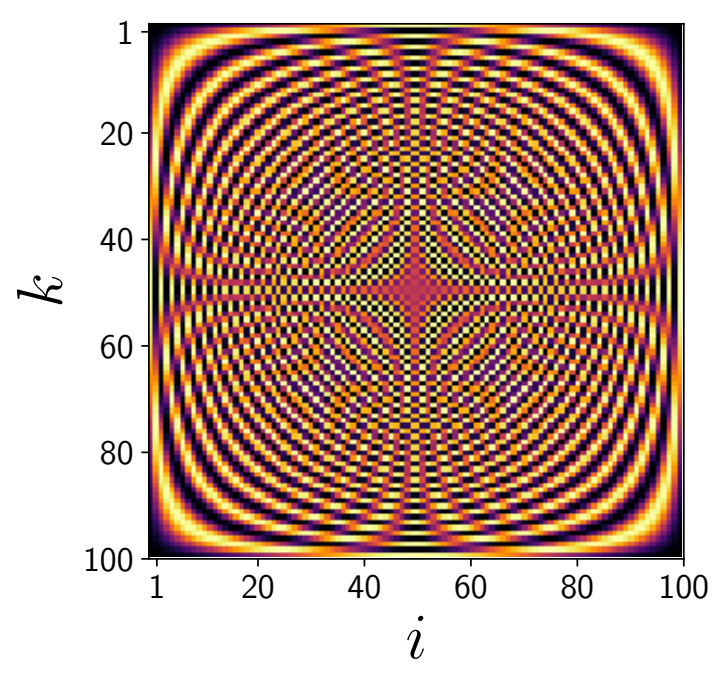

(a)

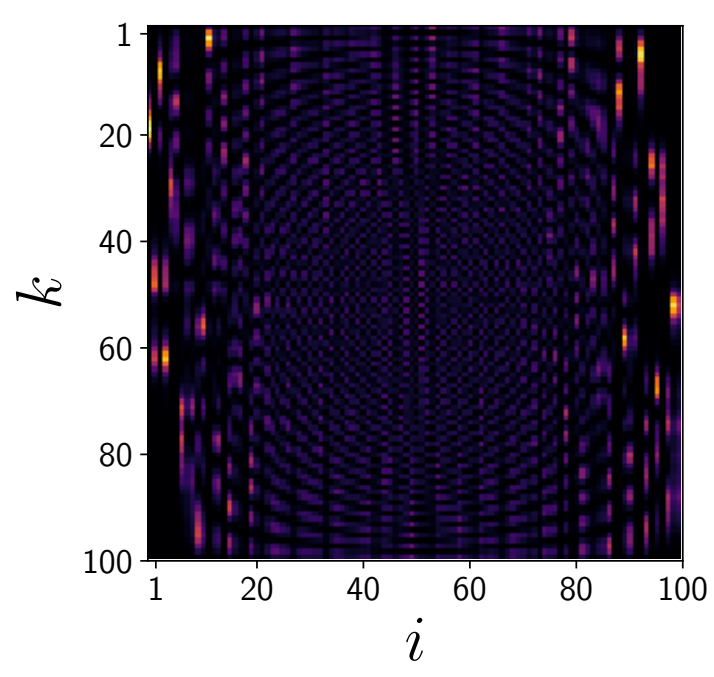

(b)

Figure 2.5: Matrix of coefficients $\left|\phi_{i}^{k}\right|^{2}$ for zero disorder (a) and for $W=0.5$ (b), for a lattice with $L=100$. In both plots, each column corresponds to an eigenvector $k$, and the rows corresponds to a site $i$. The brigthness at position $i, k$ is related to the magnitude of $\left|\phi_{i}^{k}\right|^{2}$

where $T_{n}$, called transfer matrix, is defined by

$$
T_{n}=\left(\begin{array}{cc}
E-V_{n} & -1 \\
1 & 0
\end{array}\right)
$$

Notice that $T_{n}$ is a random matrix, because one of its elements is the random variable $E-V_{n}$. By iterating Eq. (2.46) $n$ times, we obtain

$$
\left(\begin{array}{c}
\phi_{n+1} \\
\phi_{n}
\end{array}\right)=\prod_{i=1}^{n} T_{i}\left(\begin{array}{c}
\phi_{1} \\
\phi_{0}
\end{array}\right)
$$

where $\phi_{0}=0$ due to the open boundary conditions. This equation permits to calculate the behavior of the wave function $\left|\phi_{n}\right|$ for large values of $n$ based on the statistical properties of the product of random matrices $\prod_{i=1}^{n} T_{i}$. Using the Furstenberg theorem [36], one may show that for $L \rightarrow \infty$ the solutions have the form

$$
\left|\phi_{n}\right| \sim \exp \left(-\frac{\left|n-n_{0}\right|}{\xi}\right), \quad n \gg 1
$$

where $n_{0}$ is the localization center and $\xi$ is the localization length. Thus, for $W>0$, all the eigenstates are localized. 
If the dimension is three or higher, delocalized eigenstates can exist for weak disorder. Therefore, there exists a critical disorder strength above which the system undergoes a phase transition, the so called metal-insulator transition (MIT), and all the eigenstates become localized [37, 38]. 


\section{Chapter 3}

\section{Quasiperiodic Potentials}

In this chapter we will review the localization properties of two of the most well-known quasiperiodic systems, the Aubry-André-Harper model and the Fibonacci model. We will then discuss how to numerically characterize the localization of a state using the Inverse Participation Ratio.

\subsection{Aubry-André-Harper model}

The most paradigmatic example of a quasiperiodic system is the Aubry-André-Harper (AAH) model [10, 11]. The Hamiltonian is the same as in Eq. 2.1, with the on-site potential given by

$$
V_{i}=2 \lambda \cos (2 \pi b i+\theta), \quad i=1, \ldots, L
$$

where $\lambda$ is the potential strength, $b$ is a constant and $\theta$ is an arbitrary global phase. This potential is called quasiperiodic due to the fact that, when $b$ is an irrational number, the function (3.1) never repeats itself along the lattice, as illustrated in Fig. 3.1.

The AAH Hamiltonian can nowadays be realized in ultracold atoms experiments [1821]. The most common setup consists in using a bichromatic lattice, where two lasers with incommensurate wavelengths are superimposed [39]. A pedagogical introduction can be found in [40].

As discussed in section 2.5, disordered one-dimensional systems are always localized, 


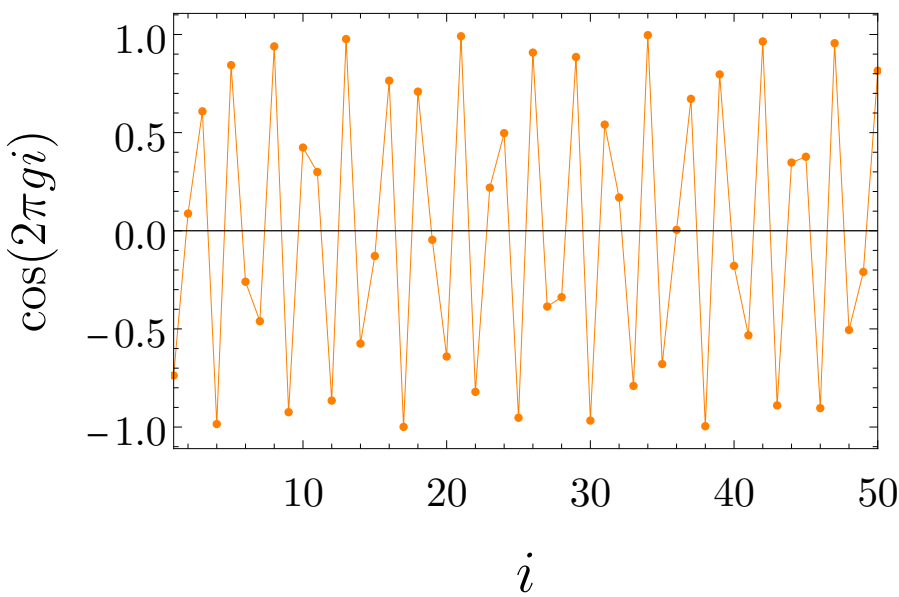

Figure 3.1: The function $2 \cos (2 \pi \varphi i)$, where $\varphi=\frac{1}{2}(1+\sqrt{5})$. The function never repeats itself for integer $i$.

while in three dimensions they exhibit a metal-insulator transition. The AAH potential, however, gives rise to very interesting localization properties, even in one dimensional chains, provided that $b$ belongs to the class of Diophantine numbers. An irrational number is said to be Diophantine if there exists $C>0$ and $r \geq 2$ such that for any rational $p / q$, with $q>0$, one has

$$
\left|b-\frac{p}{q}\right|>\frac{C}{q^{r}}
$$

When this condition is met, the model is known to go through a localization transition at the critical value $\lambda_{c}=1$ [41]. For $\lambda<1$, all the eigenstates are extended, while for $\lambda>1$ all the eigenstates are localized. The localization length, as defined in Eq. (2.49), is given by $\xi=1 / \log (\lambda)$ [10]. A common choice for $b$ in the literature, which we adopt throughout this dissertation, is the golden mean $\varphi=\frac{1}{2}(1+\sqrt{5})$.

The localization transition is depicted in Fig. 3.2, which shows the probabilities $\left|\phi_{i}\right|^{2}$ for different values of $\lambda$ for a lattice with $L=100$. As can be seen, for $\lambda<1$ the wave function is extended through all the sites, while for $\lambda=1.2$ it is extremely localized at some particular site, called the localization center. The position of this center changes with the value of $\theta$. Notice that the vertical scale in each plot is different, as the maximum value of the probability is much smaller in the delocalized phase. At the critical value $\lambda=1.0$, the overall shape of the wave function is very similar to the localized one, except 
that it exhibits some small "fringes". As we shall discuss in section 3.2, the localization length at $\lambda=1$ scales differently with the system size, compared to $\lambda>1$ : the wave function is neither extended nor localized, but critical, in a sense that shall be precisely defined in the next section.
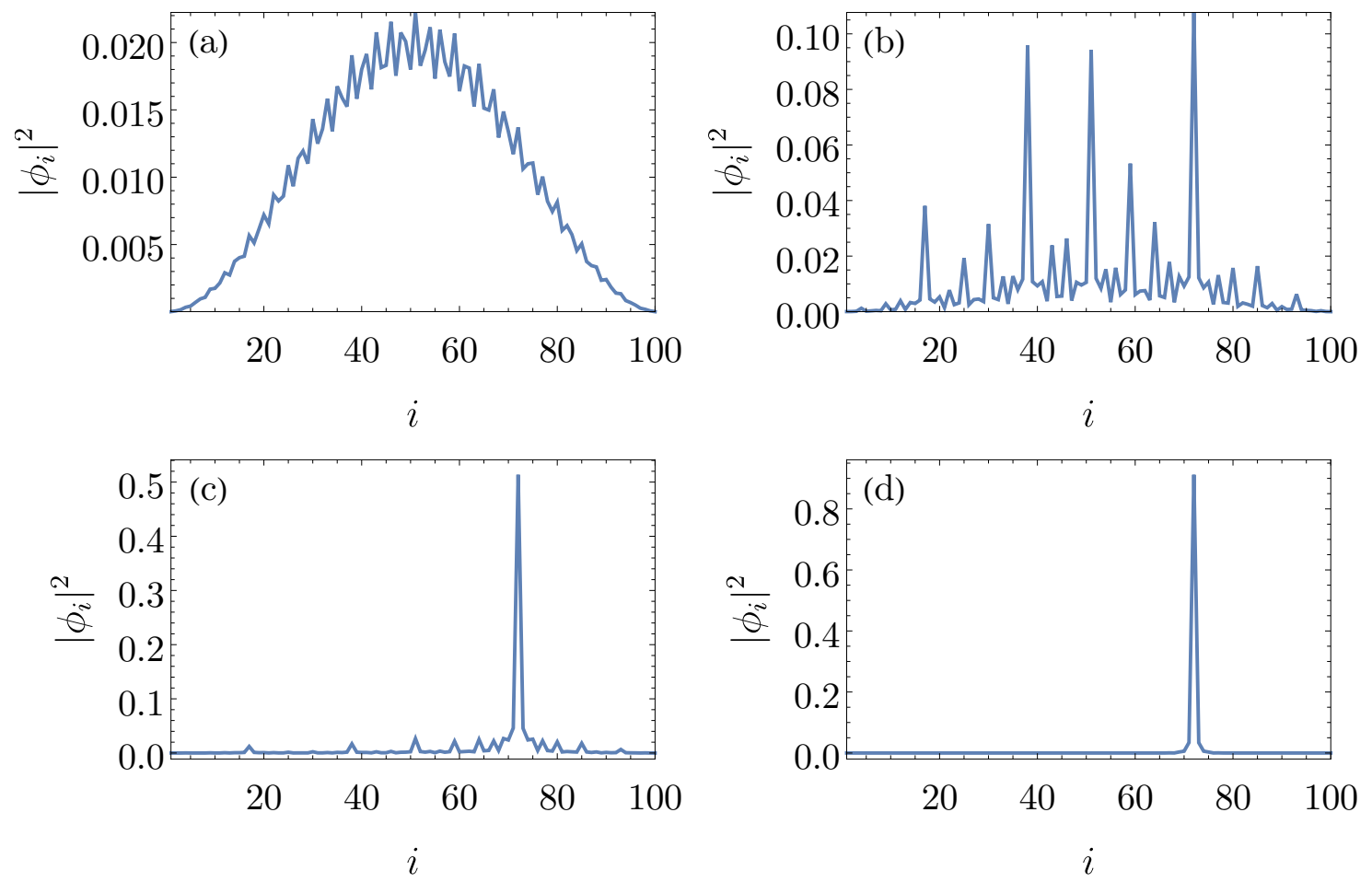

Figure 3.2: Ground-state eigenvector of the AAH model for (a) $\lambda=0.5$, (b) $\lambda=0.9$, (c) $\lambda=1.0$ and (d) $\lambda=1.2$.

The AAH also has the distinctive feature of being self-dual under a certain transformation, closely related to a discrete Fourier transform. Consider the first-quantized version of the Hamiltonian,

$$
H=-\sum_{n=1}^{L}(|n\rangle\langle n+1|+| n+1\rangle\langle n|)+\lambda \sum_{n=1}^{L} 2 \cos (2 \pi b n)|n\rangle\langle n|,
$$

where we assumed, for simplicity, periodic boundary conditions. We then define the following transformation:

$$
|k\rangle=\sum_{n=1}^{L} e^{2 \pi i b k n}|n\rangle
$$


This mapping is very similar to the discrete Fourier transform, but with an extra factor of $b$ in the exponent. The states $|k\rangle$ satisfy an orthogonality condition,

$$
\left\langle k^{\prime} \mid k\right\rangle=\sum_{n} e^{2 \pi i b\left(k-k^{\prime}\right) n}=\delta_{k, k^{\prime}}
$$

Then, applying this transformation to Eq. (3.3), one obtains

$$
H^{\prime}=-\lambda \sum_{n=1}^{L}(|k\rangle\langle k+1|+| k+1\rangle\langle k|)+\sum_{n=1}^{L} 2 \cos (2 \pi b k)|k\rangle\langle k|,
$$

which has the same structure of Eq. (3.3), except that the roles of the tunneling rate and the strength of the potential $\lambda$ are reversed. Notice that this self duality property holds for any value of $b$, not only when it is Diophantine.

This self-duality property determines the precise critical point of the localization transition, as shown in the original paper by Aubry and André [10]. If a state written in the $\{|n\rangle\}$ basis is localized, then it must be extended in the basis $\{|k\rangle\}$, according to the transformation (3.4). Thus, if the eigenstates of $H$ are localized, then the eigenstates of $H^{\prime}$ are extended. The converse is also true. Therefore, if a localization transition occurs, it must be at $\lambda=1$, which is the only parameter for which the transformation maps $H$ into itself. Notice, however, that this property does not imply that there must be a localization transition; it simply determines the critical value in case it occurs.

\subsection{Inverse Participation Ratio (IPR)}

The localization of a state can be numerically quantified trough the Inverse Participation Ratio (IPR). Given a state $|\psi\rangle=\sum_{n} \psi_{n}|n\rangle$, its IPR is defined as

$$
\operatorname{IPR}(\psi)=\sum_{n=1}^{L}\left|\psi_{n}\right|^{4}
$$

The maximum value of the IPR corresponds to a state infinitely localized at some site $n_{0}$, which can be written as $|\psi\rangle=\sum_{n} \delta_{n, n_{0}}|n\rangle$, yielding 


$$
\operatorname{IPR}(\psi)=\sum_{n=1}^{L}\left|\delta_{n, n_{0}}\right|^{4}=1 .
$$

The smallest valued is achieved when the state is completely delocalized, such as the plane waves (2.35). In this case, the IPR is given by

$$
\operatorname{IPR}(\psi)=\sum_{i=1}^{L} \frac{1}{L^{2}}=\frac{1}{L}
$$

which vanishes in the limit $L \rightarrow \infty$. Therefore, the IPR is a number ranging from $1 / L$ to 1. The more localized the state is, the closer it is to 1 .

The IPR is also closely related to the localization length. For localized states, which decay exponentially, as in Eq. (2.49), these quantities are inversely proportional and hence IPR $\sim \xi^{-1}$. Although this relation does not hold in general, the two quantities are still related, and the IPR is frequently used in the literature as proxy for the localization length.

The IPR can be used to probe the localization in the AAH model. The transition can be clearly visualized in Fig. 3.3, which shows the IPR averaged over all eigenstates as a function of $\lambda$ for different values of $L$. Notice that the transition becomes increasingly sharper as $L \rightarrow \infty$.

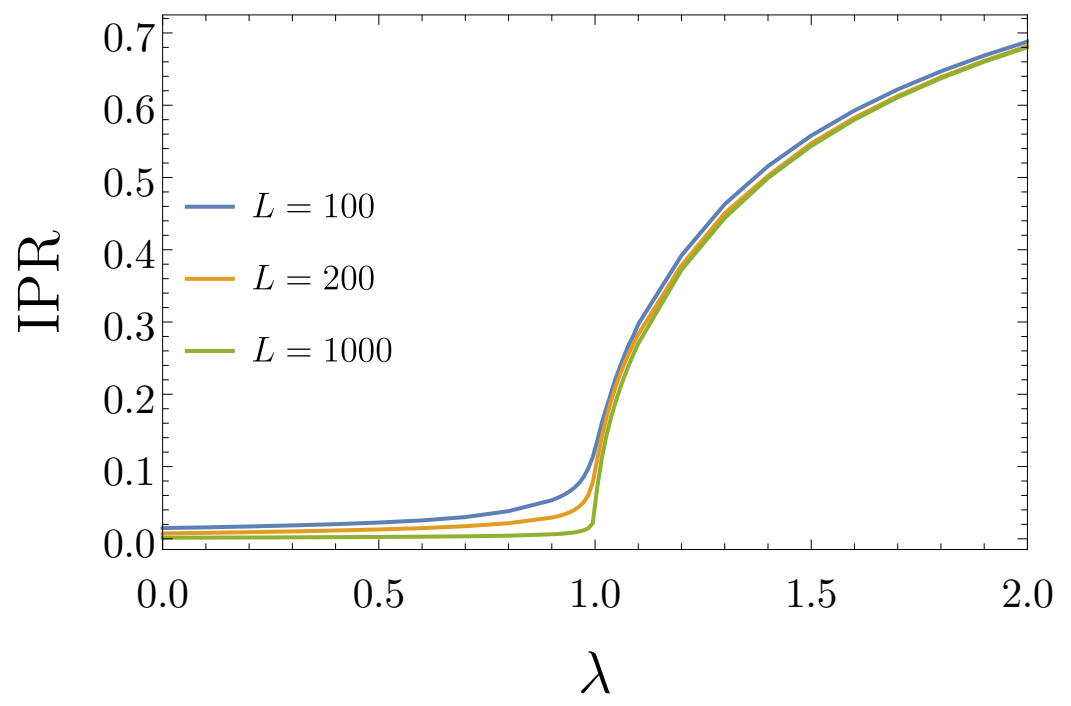

Figure 3.3: Inverse Participation Ratio (IPR) averaged of all eingestates of the AAH model, as a function of $\lambda$ for different values of $L$ and $\theta=0$.

The localized and delocalized phases can also be characterized through the scaling 
of the IPR with the system size $L$. For the extended eigenstates, the IPR scales as IPR $\sim L^{-1}$, whereas in the localized phase it remains constant with $L$, IPR $\sim L^{0}$. At criticality, the IPR exhibits an anomalous scaling, IPR $\sim L^{-\tau}$, where $0<\tau<1$. This coefficient $\tau$ can be computed, and it is related to the fractal properties of the eigenstates [42-44]. Fig. 3.4 shows the scaling of the IPR with the system size $L$ for multiple values of $\lambda$. Notice that, at criticality, the IPR scales with a different coefficient compared to the localized state. For this reason, the states at $\lambda=1$ are classified as critical, rather than localized or delocalized.

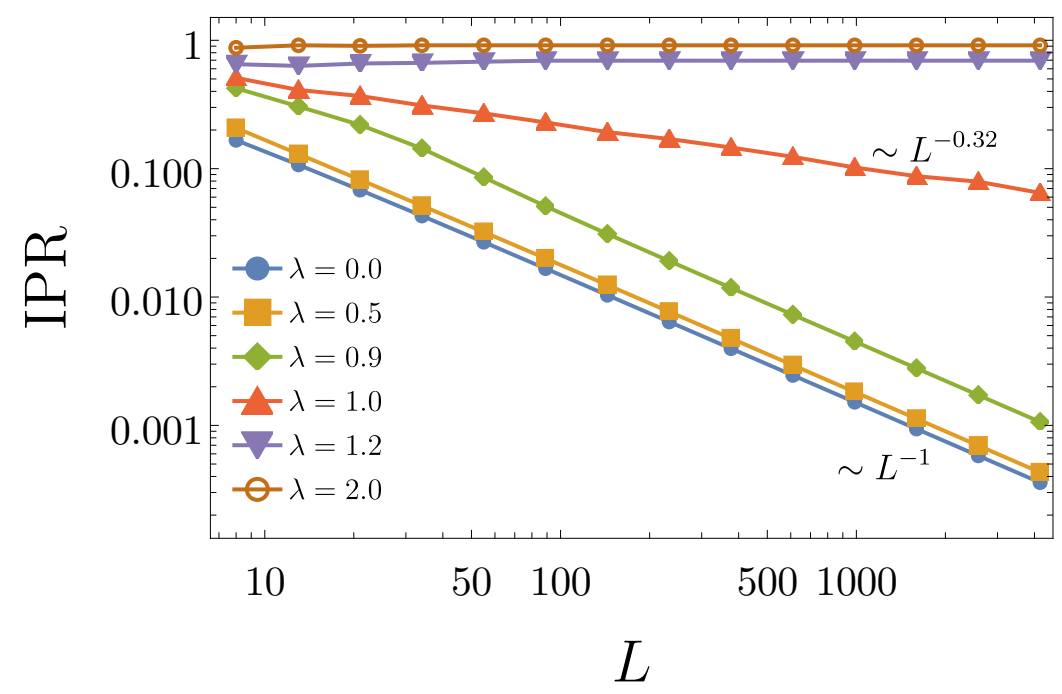

Figure 3.4: Scaling of the IPR as a function of $L$ in the AAH model, for different values of $\lambda$.

\subsection{Fibonacci model}

The second quasiperiodic system we studied in this dissertation is the Fibonacci model [12-15], which is again described by the tight-binding Hamiltonian [Eq. (2.1)]. The onsite potential of this model is constructed from a peculiar sequence of $A \mathrm{~s}$ and $B$ symbols called Fibonacci word. Starting from the strings $S_{1}=A$ and $S_{2}=A B$, each Fibonacci word is defined as the concatenation of the two previous ones,

$$
S_{n+1}=S_{n}+S_{n-1}, \quad n>2,
$$

where the operation + is to be understood as concatenation of strings. Successive appli- 
cation of this rule generate the sequence of Fibonacci words:

$$
\begin{aligned}
& S_{1}=A, \\
& S_{2}=A B, \\
& S_{3}=A B A, \\
& S_{4}=A B A A B
\end{aligned}
$$

Notice that, by construction, each word is a prefix of the following one, and also the lengths of the words form the sequence of Fibonacci numbers, that is, $\left|S_{n}\right|=F_{n}$, where $F_{n}$ is the $n^{\text {th }}$ Fibonacci number. In the limit $n \rightarrow \infty$, one may show that the Fibonacci words converge to a unique sequence

$$
S_{\infty}=A B A A B A B A A B A A B A B A \ldots,
$$

which we shall refer simpy as the Fibonacci sequence. This sequence has a simple closedform expression $[45,46]$

$$
S_{\infty, i}= \begin{cases}A & \text { if }\lfloor(i+1) / \varphi\rfloor \neq\lfloor i / \varphi\rfloor, \\ B & \text { otherwise, }\end{cases}
$$

where $\lfloor\cdot\rfloor$ is the floor function. The Fibonacci on-site potential is then constructed by associating each element of the sequence with a position in the lattice and replacing the letters $A$ with some value $V_{A}$ and the letters $B$ with a value $V_{B}$, as illustrated in Fig. 3.5.

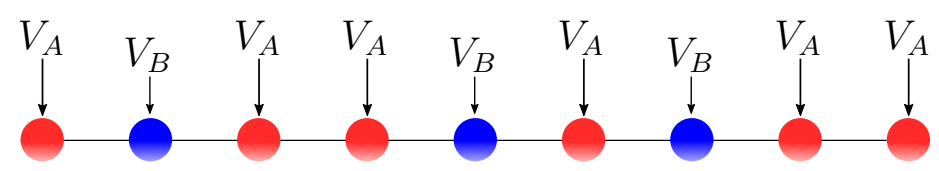

Figure 3.5: Schematic representation of the construction of the Fibonacci potential. Each element in the sequence is associated with a site in the quantum chain. The letter $A$ is replaced by a value $V_{A}$ and the letter $B$ by a value $V_{B}$. In this dissertation, we choose $V_{A}=\lambda$ and $V_{B}=0$.

In this dissertation we will chose the values $V_{A}=\lambda$ and $V_{B}=0$, where $\lambda$ is a real parameter. The difference $\lfloor(i+1) / \varphi\rfloor-\lfloor i / \varphi\rfloor$ is either 0 or 1 , therefore, using Eq. 3.13, we can write 


$$
V_{i}=\lambda\left(\left\lfloor\frac{i+1}{\varphi}\right\rfloor-\left\lfloor\frac{i}{\varphi}\right\rfloor\right) .
$$

This is the expression we used in our numerical simulations to construct the Hamiltonian.

In this definition of the on-site potential, the first element of the Fibonacci potential is associated with the first site in the chain. This, however, is not really a restriction. For a system with $L$ sites, any subsequence of length $L$ can be used. When numerical simulations are perfomed, the computed quantities can be averaged over these different realizations of the potential, which in general produces smoother curves. A natural question is, then, how many subsequences of length $L$ exist. If the sequence were completely random, there would be an infinite number of subsequences of a given length. In the Fibonacci sequence, however, there are only $L+1$ subsequences of length $L$, which occur with the same rate along the infinite sequence. For example, when $L=2$, only the subsequences $A A, A B, B A$ can be found: the configuration $B B$ never occurs.

All the subsequences off the Fibonacci word can be constructed using the algorithm described in Ref. [46]. In this dissertation, we mostly used the single sample version, defined in Eq. 3.14, except when explicity mentioned otherwise. All the samples of the potential yield the same qualitative behaviour, although the transport coefficients might differ slightly. A more careful discussion of these differences is done in Ref. [17].

The Fibonacci sequence can also be constructed using a myriad of alternative procedures, which we now briefly discuss for completeness. For example, one might use the substitution rule

$$
\left\{\begin{array}{l}
A \rightarrow A B \\
B \rightarrow A .
\end{array}\right.
$$

Starting from the string " $A$ ", at each application of the rule, each letter $A$ is replaced by $A B$ and each letter $B$ is replaced by $A$, generating the same sequence as before. This rule is also frequently written in matrix notation,

$$
\left(\begin{array}{l}
A \\
B
\end{array}\right) \rightarrow\left(\begin{array}{ll}
1 & 1 \\
1 & 0
\end{array}\right)\left(\begin{array}{l}
A \\
B
\end{array}\right)
$$

where the sum in the matrix product is to be understood as string concatenation. Many 
properties of the system can be derived from the eigenvectors and eigenvalues of this matrix, which is called the substitution or inflation matrix. Notice that at each iteration of the rule the total number of $A$ 's in the sequence is given by the length of the preceding word, which is a Fibonacci number, that is

$$
N_{n}^{A}=N_{n-1}^{A}+N_{n-1}^{B}=\left|S_{n-1}\right|=F_{n-1}, \quad n>1 .
$$

Similarly, the total number of $B$ 's equals the number of $A$ 's in the preceding word, which per the equation above is also a Fibonacci number,

$$
N_{n}^{B}=N_{n-1}^{A}=F_{n-2}, \quad n>1
$$

Therefore, the ratio of $A$ 's and $B$ 's in the sequence equals the ratio between two consecutive Fibonacci numbers, which famously converges to the golden mean in the limit $n \rightarrow \infty$,

$$
\lim _{n \rightarrow \infty} \frac{N_{n}^{A}}{N_{n}^{B}}=\lim _{n \rightarrow \infty} \frac{F_{n}}{F_{n+1}}=\varphi .
$$

This fact shows that the sequence cannot be periodic, since otherwise the ratio between the two letters would have to be a rational number

There is also another interesting way of constructing the Fibonacci word, based on a geometrical interpretation, as illustrated in Fig. 3.6. First, a line with slope $1 / \varphi$ is drawn in a unit grid, starting from the origin. The Fibonacci sequence can then be read off by following this line and checking the intersections with the grid. When a vertical grid line is crossed, the letter $A$ is attached to the sequence, while an intersection with a horizontal grid line corresponds to the letter $B$. Sequences constructed this way are known as "cutting sequences". Therefore, the Fibonacci word is characterized by a cutting sequence of the line with slope $1 / \phi$.

All the eigenstates of the Fibonacci model are critical [12, 13, 47], similarly to the AAH at $\lambda=1$. This means that IPR exhibits an anomalous scaling of the form

$$
\mathrm{IPR} \sim L^{-\tau(\lambda)}
$$




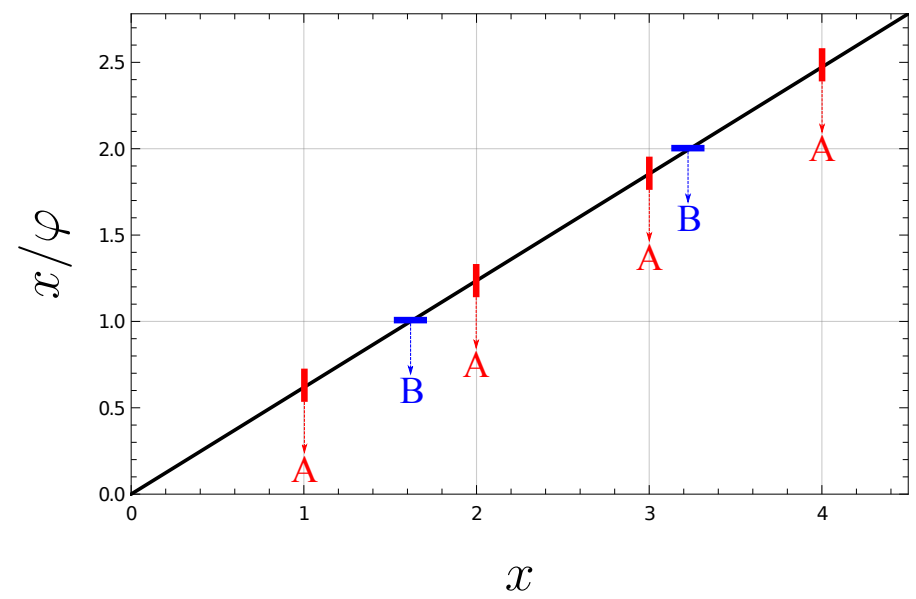

Figure 3.6: Construction of the Fibonacci chain by cutting a sequence of the line with slope $1 / \phi$. When the line with slope $1 / \phi$ crosses a vertical grid line, the letter $A$ attached to the sequence, while an intersection with a horizontal grid line corresponds to a letter $B$.

The coefficient $\tau(\lambda)$ is related to the fractal properties of the eigenstates [42, 43, 48]. In Fig. 3.7, we show the scaling of the IPR for different values of $\lambda$, averaged over all eigenstates. In Fig. 3.7 (a), on the left, the system sizes used are Fibonacci numbers. Curiously, when every other Fibonacci number is skipped in the plot, the resulting curve is virtually a straight line. We suspect that this is related to the fractal and number theoretical properties of the eigenstates.
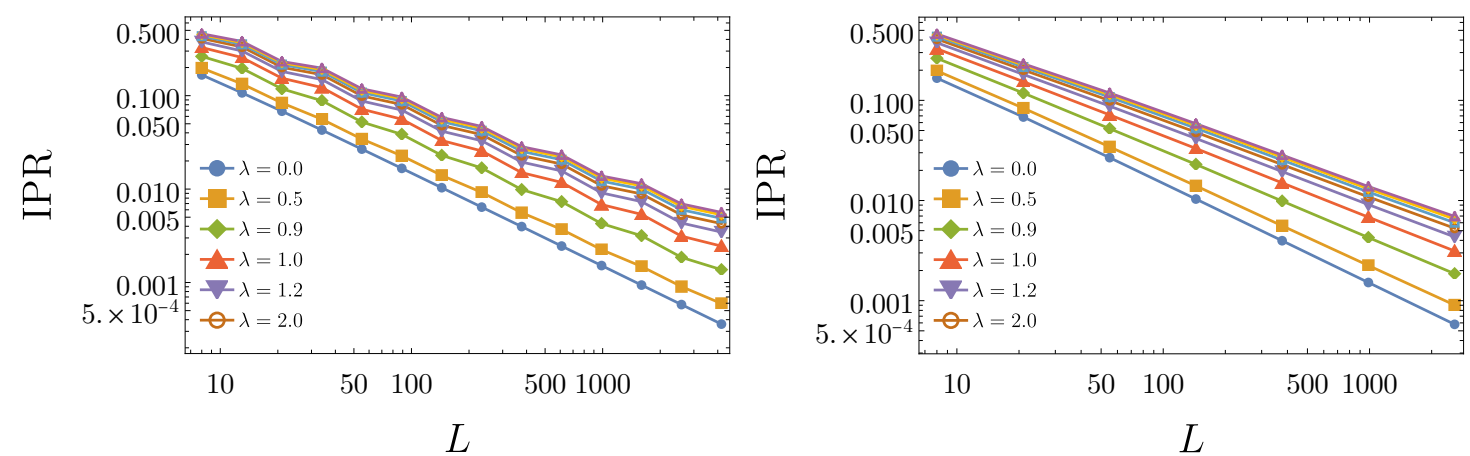

Figure 3.7: (a) Scaling of the IPR with $L$ in the Fibonacci model for different values of $\lambda$, where values of $L$ are Fibonacci numbers. (b) Scaling of the IPR with $L$ in the Fibonacci model, skipping every other Fibonacci number.

Notice that the value of $\tau$ decreases with $\lambda$, which is shown in Fig. 3.8 (a). Moreover, for a fixed value of $L$, the mean IPR of the eigenstates increases continuously with $\lambda$, which means that the eigenstates become more localized. This is already an indicative that the transport regime of the Fibonacci model varies continuously with $\lambda$, as we mentioned 
in the introduction.
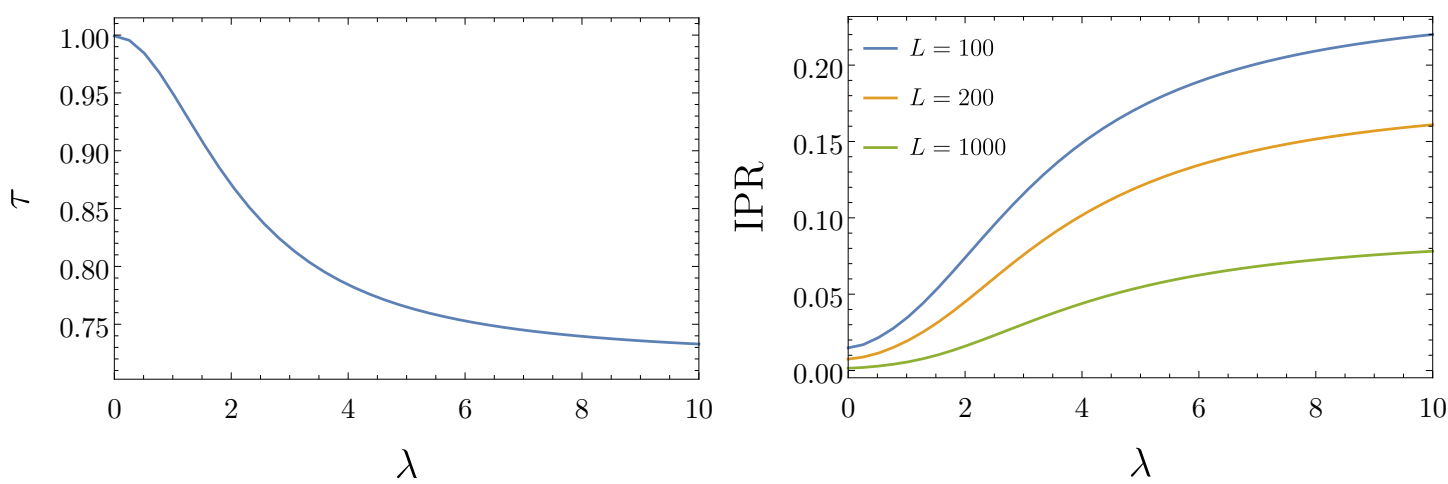

Figure 3.8: (a) Dependence of the coefficient $\tau$ with $\lambda$, defined in Eq. (3.20), in the Fibonacci model. (b) IPR averaged over all the eigenstates of the Fibonacci number with respect to $\lambda$. In each curve, the value of $L$ is fixed. 


\section{Chapter 4}

\section{Coherent transport}

In this chapter, we will study transport properties of the fermionic systems presented in Chapter 3, in a closed system scenario. We will do so by computing the time evolution of the mean squared displacement of the wave function. This, perhaps, is one of the simplest frameworks one may conceive to probe the transport regime, but it already reveals all the surprising features of the quasiperiodic chains. In section 4.1, we will discuss the equation of motion of the system and provide an efficient Mathematica code used in our numerical simulations. Then, in section 4.2, we will the describe the framework we used to classify the transport. In the remaining sections, we will discuss the unitary transport properties of the AAH and Fibonacci models.

\subsection{Unitary time evolution}

When the system is isolated, its time evolution is dictated by the Schrödinger equation,

$$
\frac{\partial}{\partial t}|\psi(t)\rangle=-i \mathcal{H}|\psi(t)\rangle,
$$

where we fixed $\hbar=1$. In this chapter, we will always choose an initial state completely localized at some site $n_{0}$ :

$$
|\psi(t)\rangle=\sum_{n} \delta_{n, n_{0}}|n\rangle,
$$

which is a single particle state. As discussed in chapter 2, the number of particles is 
conserved during a unitary evolution. Therefore, all the subsequent states will have a single particle, and can thus be expanded as

$$
|\psi(t)\rangle=\sum_{n} \psi_{n}(t)|n\rangle
$$

We will now derive an equation of motion for the coefficients $\psi_{n}$. After a substitution of this expansion in Eq. 4.1, one obtains

$$
\frac{\partial}{\partial t}|\psi(t)\rangle=-i \sum_{m} \sum_{i j} H_{i j} c_{i}^{\dagger} c_{j} \psi_{m}(t)|m\rangle
$$

The differential equations for the coefficients $\psi_{n}$ are then obtained by contracting on the left with $\langle n|$ and using the fact that $\left\langle n\left|c_{i}^{\dagger} c_{j}\right| m\right\rangle=\delta_{i n} \delta_{j m}$, which results in

$$
\frac{\partial}{\partial t} \psi_{n}(t)=-i \sum_{n m} H_{n m} \psi_{m}(t) .
$$

Therefore, as expected, when the many-body system is restricted to single-particle states, its time evolution is equivalent to that of the $L$ level system, dictated by the $L \times L$ matrix $H$.

Defining the vector of coefficients $\boldsymbol{\psi}=\left(\psi_{1}, \ldots, \psi_{L}\right)$, Eq. (4.5) can be written matricially as

$$
\frac{\partial}{\partial t} \boldsymbol{\psi}(t)=-i H \boldsymbol{\psi}(t)
$$

which has the formal solution $\boldsymbol{\psi}(t)=e^{-i H t} \boldsymbol{\psi}(0)$. Using the decomposition $H=S \mathcal{E} S^{\dagger}$, the time evolution operator can be computed as

$$
e^{-i H t}=S e^{-i \mathcal{E} t} S^{\dagger}
$$

where $e^{-i \mathcal{E} t}=\operatorname{diag}\left(e^{-i \epsilon_{1} t}, \ldots, e^{-i \epsilon_{L} t}\right)$.

A typical method to numerically integrate Eq. (4.5) is to compute the time propagator $U(\tau)=e^{-i H \tau}$, where $\tau$ is a fixed time step, and then successively obtain the state at each step by the recursive relation 


$$
\boldsymbol{\psi}\left(t_{k+1}\right)=U(\tau) \boldsymbol{\psi}\left(t_{k}\right)
$$

where $t_{k}=k \tau$. This procedure, however, has some major flaws. Firstly, numerical errors accumulate at each matrix multiplication. Secondly, the time steps $t_{k}$ are evenly spaced in the linear scale. However, the classification of the transport regime will require the simulation of the system for very large times, and thus all the curves obtained will be displayed in logarithmic scale. Therefore, if the time steps are linearly spaced, the small time region becomes very clogged with data points, while the long time region becomes sparse. For this reason, it is convenient to choose time steps $t_{n}$ that are evenly spaced in the logarithmic scale. To do so, we simply use Eq. (4.7) to compute the wave function for any $t_{n}$ :

$$
\boldsymbol{\psi}\left(t_{n}\right)=S e^{-i \mathcal{E} t_{n}} S^{\dagger} \boldsymbol{\psi}(0)
$$

In this procedure, the matrix $H$ has to be diagonalized a single time. At each time step, only the matrix exponential $e^{-\mathcal{E} t_{n}}$ is computed. Since the matrix $\mathcal{E}$ is diagonal, this is equivalent to exponentiating $L$ complex numbers, which can be done very efficiently. Moreover, since the initial state is fixed, one matrix multiplication can be avoided at each time step by previously defining $\phi=S^{\dagger} \boldsymbol{\psi}(0)$, which results in

$$
\boldsymbol{\psi}\left(t_{n}\right)=S e^{-i \mathcal{E} t_{n}} \boldsymbol{\phi}
$$

In Mathematica, this procedure can be made even more efficient by exploiting the language's vectorization features. Instead of using a loop to compute each time step, all the operations can be done simultaneously using matrix notation. Consider a matrix $\mathcal{T}$ defined by

$$
\mathcal{T}_{i j}=e^{-i \epsilon_{i} t_{j}}
$$

This matrix can be found efficiently in Mathematica by computing the outer product of the vectors $\left(\epsilon_{1}, \ldots, \epsilon_{L}\right)$ and $\left(t_{1}, \ldots, t_{L}\right)$ and exponentiating each element. At each time step $t_{n}$, the coefficients $\psi_{i}\left(t_{n}\right)$ are then given by 


$$
\psi_{i}\left(t_{j}\right)=S \mathcal{T}_{i j} \phi_{i}
$$

which can be written matricially as

$$
\Psi=S(\phi * \mathcal{T})
$$

where the notation $(\phi * \mathcal{T})$ means that each column of $\mathcal{T}$ is multiplied elementwise by $\phi$ and $\Psi$ is a matrix containing the wavefunctions at all times on its columns:

$$
\Psi=\left(\begin{array}{ccc}
\psi_{1}\left(t_{1}\right) & \ldots & \psi_{1}\left(t_{N}\right) \\
\psi_{2}\left(t_{2}\right) & \ldots & \psi_{2}\left(t_{N}\right) \\
\vdots & & \vdots \\
\psi_{L}\left(t_{2}\right) & \ldots & \psi_{L}\left(t_{N}\right)
\end{array}\right)
$$

In our simulations, this procedure was implemented using the following Mathematica code:

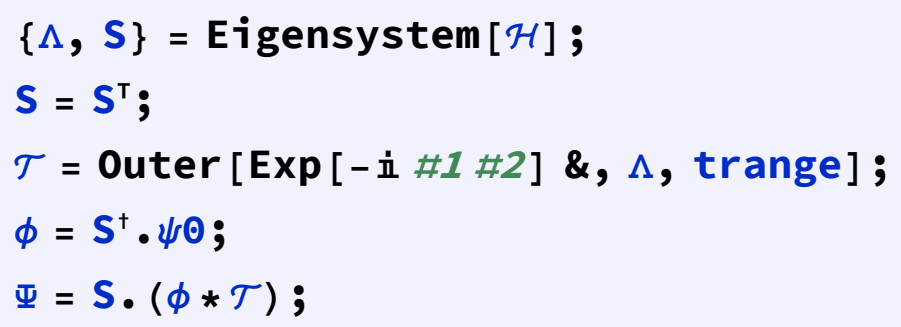

Since all the operations are vectorized, with no loop explicity written, all the operations are delegated by Mathematica to its internal linear algebra implementations, with virtually no overhead, and are thus performed very efficiently.

\subsection{Classification of the transport regime}

Consider the classical example of a particle performing a random walk in a 1D lattice The particle starts at the position $x=0$, and at each time step $\Delta t$, moves one unit to the right or to the left, with equal probability. After $N$ time steps, corresponding to the time $t=N \Delta t$, the variance of its position is given by 


$$
\left\langle(\Delta x)^{2}\right\rangle=D t^{2}
$$

where $D=1 / \Delta t$. Therefore, the standard deviation of $x$ is proportional to $t$. This behaviour is typically known as diffusion. We will import this idea to quantum mechanics and build a simple framework to probe the transport properties of our systems.

We will always assume that the particle is initially localized at the central site of the chain, $n_{0}$, thus

$$
\psi_{n}(0)=\delta_{n, n_{0}}
$$

Using the floor function, $n_{0}$ can be written as $n_{0}=\left\lfloor\frac{L+1}{2}\right\rfloor$. After $t>0$, the system is left to evolve unitarily, so its wavefunction starts to spread trough the chain, as illustrated in Fig. 4.1.

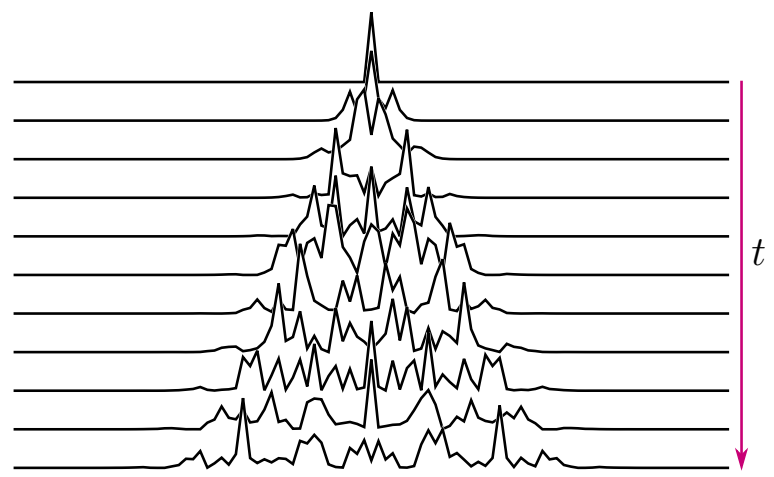

Figure 4.1: Pictorial illustration of the spreading of the wavefunction in a unitary dynamics starting from the central site.

The spreading of the wave function can be quantified via the root mean square deviation (RMSD) with respect to the central site, defined by

$$
\sqrt{(\Delta x)^{2}}=\sqrt{\sum_{n=1}^{L}\left(n-n_{0}\right)^{2}\left|\psi_{n}\right|^{2}} .
$$

The scaling of the RMSD with time is not necessarily diffusive, as in Eq. 4.15. In general, it follows

$$
\sqrt{(\Delta x)^{2}} \sim t^{\alpha}
$$


where $\alpha$ is a positive constant, which we call transport coefficient. The transport regime of the system is then classified depending on the value of $\alpha$. If $\alpha=1$, the transport is classified as ballistic, and if $\alpha=1 / 2$ it is diffusive. Other values of $\alpha$ correspond to anomalous transport, which can be further classified in subdiffusive, for $\alpha<1 / 2$, and superdifffusive, for $1 / 2<\alpha<1$. The extreme case of $\alpha=0$ corresponds to the absence of transport, which happens for localized systems. These classifications are summarized in table 4.1.

Table 4.1: Classification of the coherent transport regime using the value of $\alpha$.

\begin{tabular}{cc}
\hline Transport regime & Transport coefficient \\
\hline Localized & $\alpha=0$ \\
Subdiffusive & $\alpha<\frac{1}{2}$ \\
Diffusive & $\alpha=\frac{1}{2}$ \\
Ballistic & $\alpha=1$ \\
\hline
\end{tabular}

The procure to extract the coefficient $\alpha$ is illustrated in Fig. 4.2. We start by plotting $\sqrt{(\Delta x)^{2}}$ versus $t$ in logarithm scale. In the short scale range, the system behaves approximately ballistic. After a finite time, the wavefunction reaches the border of the system, and $\sqrt{(\Delta x)^{2}}$ starts to oscillate. For this reason, one must identify an intermediate time scale where the scaling (4.18) is obeyed. In Fig. 4.2, this region is highlighted in blue. After restricting the data points to this region, we simply fit a linear regression of the form

$$
\log \left(\sqrt{(\Delta x)^{2}}\right)=\alpha \log t+C
$$

The value of the intercept $C$ is irrelevant for our purposes.

\subsection{Transport properties with zero potential}

As a first example, consider the case in which the on-site potential is constant or, without loss of generality, identically zero. In this case, the system will correspond simply to a standard free fermion hopping on a regular lattice. As we stressed in the introduction, 


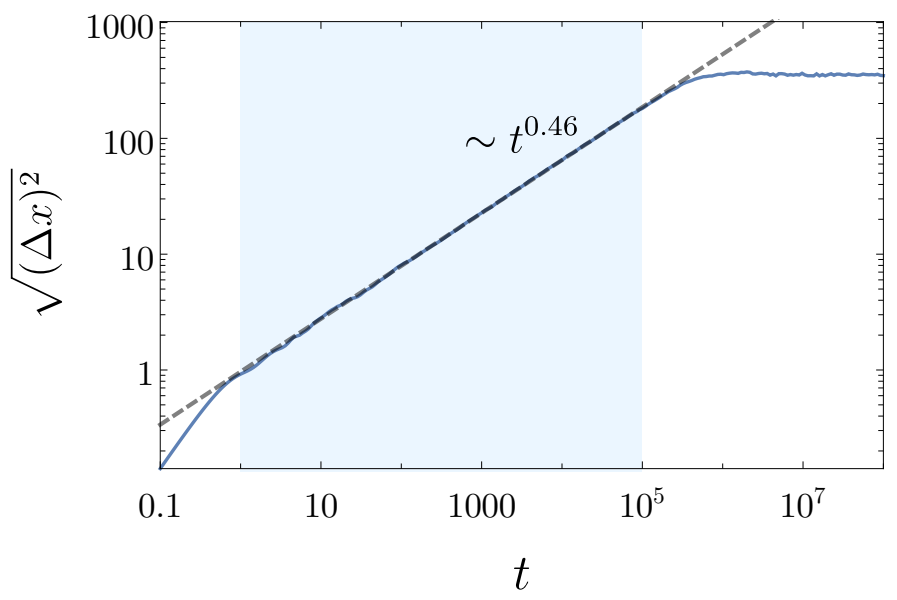

Figure 4.2: Illustration of the procedure used to computed the coefficient $\alpha$ in Eq. (4.18). This particular example corresponds to the Fibonacci model for $L=2001$ and $\lambda=4.0$, averaged over 50 configurations of the Fibonacci word. In this example, the transport is subdiffusive, with $\alpha=0.46$.

it is widely known that this model is ballistic. This can be verified in Fig. 4.3, in which we show the scaling of the RMSD in a unitary time evolution. As can be seen, for finite times, the RMSD scales perfectly proportional to $t$, until the wave function reaches the border of the system.

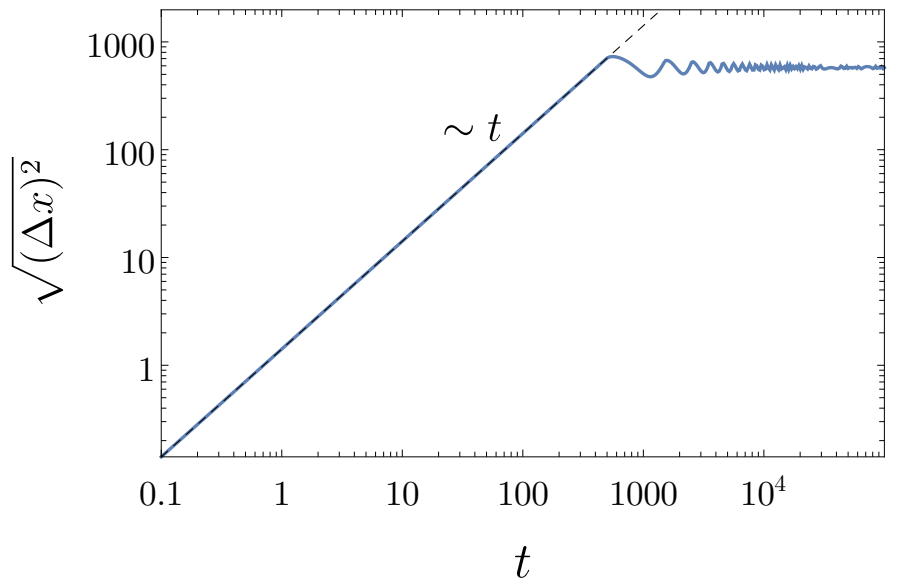

Figure 4.3: Scaling of the RMSD with $V_{i}=0$ for all $i$, for a chain with $L=2001$, starting from a state perfectly localized in the center site.

Using Eq. 4.1, the time evolution of the RMSD can also be explicitly computed, both for periodic and open boundary conditions. However, these calculations are somewhat lengthy and divert from the focus of this dissertation, we will focus instead only on the analogy with a quantum free particle. 
Assume, for simplicity, that the system is very large, and thus the boundary conditions can be ignored. In this case, the tight-binding chain is the lattice (discrete) analog of a simple free particle. The Hamiltonian of a free particle moving in one dimension is given by

$$
\mathcal{H}=\frac{p^{2}}{2 m} \text {. }
$$

Consider that, at $t=0$, the particle is described by a Gaussian wave packet centered at the origin, which corresponds to a Gaussian probability distribution of the form

$$
|\psi(x, 0)|^{2}=\frac{1}{\sqrt{2 \pi \sigma^{2}}} e^{-x^{2} / 2 \sigma^{2}}
$$

where $\sigma^{2}$ is the initial variance.

The probability distribution at arbitrary times can be obtained by solving Schrödinger's equation, and it remains Gaussian,

$$
|\psi(x, t)|^{2}=A_{t} \exp \left[-\frac{x^{2}}{2\left(\sigma^{2}+\frac{t^{2}}{4 m^{2} \sigma^{2}}\right)}\right],
$$

where $A_{t}$ is a time-dependent normalization factor. Thus, by inspection, the variance of the distribution at time $t$ is given by

$$
\left\langle(\Delta x)^{2}\right\rangle=\sigma^{2}+\frac{t^{2}}{4 m^{2} \sigma^{2}} .
$$

Therefore, in the large time limit, the RMSD is simply proportional to $t$,

$$
\sqrt{\left\langle(\Delta x)^{2}\right\rangle} \sim \frac{t}{2 m \sigma},
$$

which characterizes ballistic transport.

\subsection{Transport properties of the AAH model}

The localization transition of the AAH model is clearly reflected in its transport properties, as can be seen in Fig. 4.4, which shows the scaling of the RMSD with $t$ for different 
values of $\lambda$. For $\lambda<1$, when all the eigenstates of the system are extended, the transport is ballistic. When $\lambda>1$, which corresponds to the localized region, there is almost no spreading of the wave function, and the RMSD quickly reaches a plateau. At the critical value $\lambda=1$, the transport is very close to diffusive, but the particular value of $\alpha$ depends on the phase $\theta$. When the RMSD is averaged over $\theta$, which is the case shown in Fig. 4.4, the transport is perfectly diffusive.
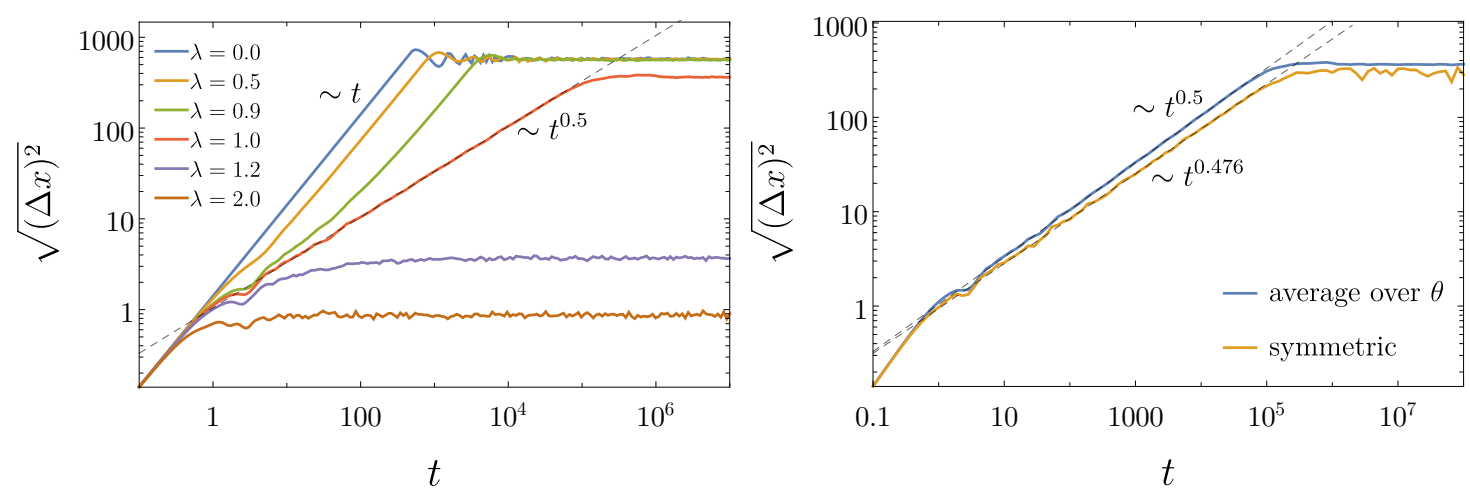

Figure 4.4: (Left) Scaling of the RMSD with time in the AAH model for a lattice with $L=2001$, starting with an initial state perfectly localized at the central site. All the values were averaged over 100 values of $\theta$, evenly spaced between 0 and $\pi$. (Right) The blue curve shows the scaling of the RMSD in the AAH potential averaged of $\theta$. The orange curve shows the scaling for the AAH potential that is chosen to be symmetrical with respect to the central site. In both curves $L=2001$ and the initial state is perfectly localized at the central site.

In Fig. 4.4 (b), we show a comparison between this averaged case and a potential that is symmetrical with respect to the central site, corresponding to $\theta=\lfloor(L+1) / 2\rfloor$. In the symmetrical case, the transport is slightly subdiffusive at the critical point, with $\alpha=0.476$. The same coefficient has been previously reported in [17].

The localization transition of the AAH model is also illustrated in Fig. 4.5, which shows the dependence of the coefficient $\alpha$ with $\lambda$, computed for a chain with $L=2001$. For $\lambda<1$, the transport is ballistic, so $\alpha=1.0$, while for $\lambda>1$ the transport is absent, thus $\alpha=0$. In this figure, the transition is not sharp because the coefficients were computed for a system with finite size. Similarly to what happens with the IPR, shown in Fig. 3.4, the transition gets sharper as $L$ increases, and only becomes discontinuous in the thermodynamic limit. 


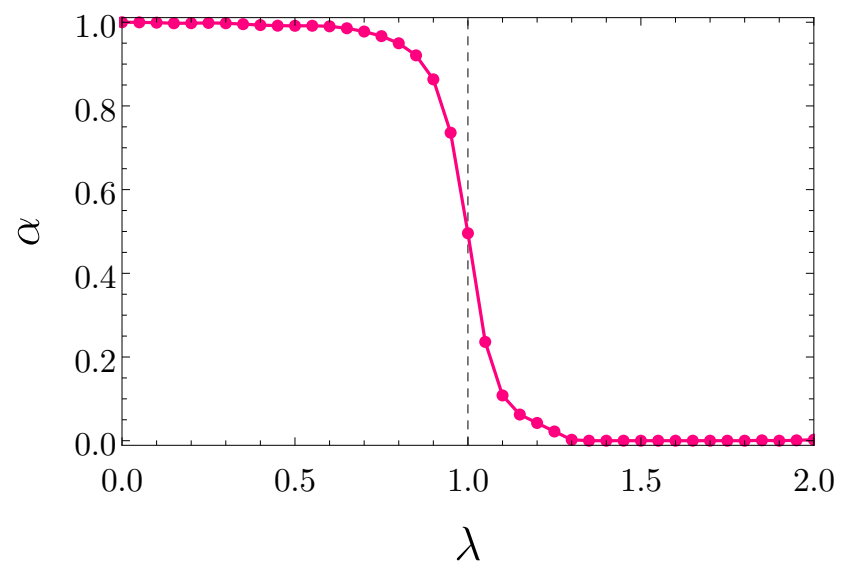

Figure 4.5: Fitted coefficient $\alpha$ as a function of $\lambda$ in the AAH model for a lattice with $L=2001$, with the initial state perfectly localized at the central site.

\subsection{Transport properties of the Fibonacci model}

As we mentioned in the introduction, the most striking feature of the Fibonacci model is its ability to exhibit any kind of transport regime, depending on the value of $\lambda$. This can be seen in Fig. 4.6, which shows the dependence of the coefficient $\alpha$ with $\lambda$, for a chain with $L=2001$. Starting from ballistic transport, at $\lambda=0$, corresponding to zero potential, the coefficient $\alpha$ decreases continuously as $\lambda$ is increased. The diffusive regime is achieved at approximately $\lambda \approx 3$. When $\lambda$ is below this value, the transport is subdiffusive, and when it is above the transport is superdiffusive. Even though this property of the Fibonacci model is known since the 1980s [15], we still find it quite remarkable, as it directly confronts the idea that free fermion chains should be ballistic.

Fig. 4.7 shows the scaling of RMSD for increasing values of $\lambda$. As can be seen, the slope of each curve decreases with increasing $\lambda$. For $\lambda=2.0$, for example, the transport is superdiffusive, while for $\lambda=4.0$ it is subdiffusive. This curve was generated by performing an average over 50 realizations of the Fibonacci word, which results in a smooth scaling for the RMSD. Notwithstanding, the transport coefficients agree with the single sample case [17]. 


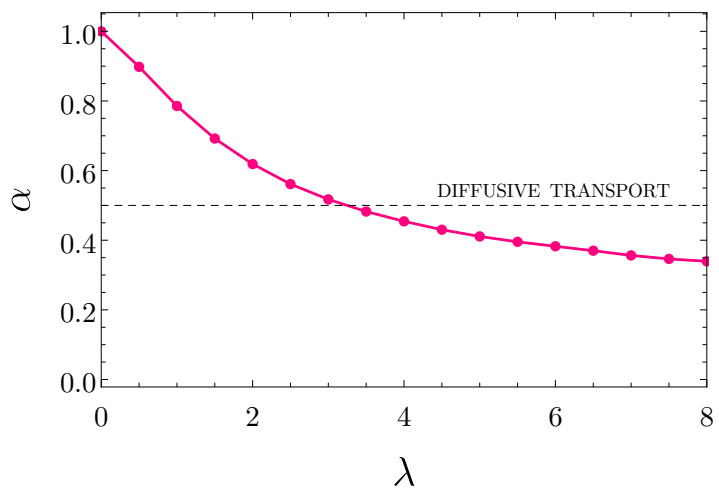

Figure 4.6: Dependence of the coefficient $\alpha$ with $\lambda$. The coefficient decreases continuously when $\lambda$ is increased. The dashed curved indicates diffusive transport, $\alpha=0.5$, which occurs for $\lambda \approx 3$.

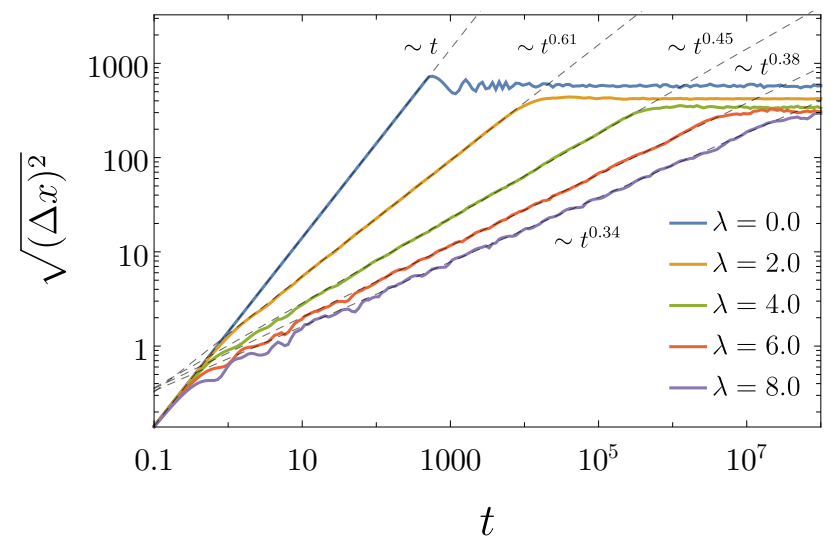

Figure 4.7: Scaling of the RMSD in the Fibonacci model for increasing values of $\lambda$, for a chain with $L=2001$, starting from a state localized at the central site. In this particular plot, all the curves were averaged over 50 samples of the Fibonacci potential. 


\section{Chapter 5}

\section{Open quantum systems}

In nature, no system can exist in perfect isolation, whether it is classical or quantum. The notion of "closed system" is only an idealization, as there is always some kind of interaction with the environment. It is frequent that these interactions can be neglected, but many physical phenomena, such as the spontaneous emission of photons, can only be explained when they are taken into account. In this chapter, we shall briefly explain the framework to describe the evolution of an open quantum system, which we will later apply to boundary-driven spin chains.

\subsection{The Lindblad master equation}

When a system is isolated, its time evolution is dictated by the Von Neumann's equation,

$$
\frac{\mathrm{d} \rho}{\mathrm{d} t}=-i[\mathcal{H}, \rho]
$$

where $\rho$ is the system's density matrix. However, when the system is put in contact with an environment and its thus subject to dissipation, this equation is no longer valid. The dynamics of an open system will therefore not be unitary. Notwithstanding, it must still be physical. That is, it must take density matrices into density matrices. Maps of this form are called Completely Positive and Trace Preserving (CPTP). We will not enter into the details of the basic mathematical structure of CPTP maps, which can be found e.g. in $[49,50]$. Instead, we will focus on the specific case of time-local (Markovian) master 
equations, which form a subset of CPTP maps. The basic intuition is to augment Eq. (5.1) with an additional term, $\mathcal{D}(\rho)$, describing the contact with the reservoir; i.e.,

$$
\frac{\mathrm{d} \rho}{\mathrm{d} t}=-i[\mathcal{H}, \rho]+\mathcal{D}(\rho)
$$

As shown in [51, 52], there exist a generic structure for $\mathcal{D}(\rho)$, which ensures that the dynamics is always CPTP. Namely, when it has the form

$$
\mathcal{D}(\rho)=\sum_{k} L_{k} \rho L_{k}^{\dagger}-\frac{1}{2}\left\{L_{k}^{\dagger} L_{k}, \rho\right\}
$$

where $\left\{L_{k}\right\}$ is an arbitrary set of operators acting on the system, called jump operators. To make the notation simpler, it is convenient to define

$$
D[L]=L \rho L^{\dagger}-\frac{1}{2}\left\{L^{\dagger} L, \rho\right\}
$$

thus $\mathcal{D}(\rho)=\sum_{k} D\left[L_{k}\right]$.

The specific structure of the $L_{k}$ depends not only on the system, but also on the type of process being modeled. The simplest example is the contact with a standard thermal bath. However, in the quantum domain, other types of evolution are also possible, such as the so-called dephasing dynamics, which does not affect populations, but only suppresses coherences. Often, the form of $\mathcal{D}(\rho)$ is obtained from a microscopic derivation, starting from a global model of system plus environment. Here we shall not pursuit this path, as it would deviate significantly from the scope of this dissertation. Instead, for the remainder of this chapter, we will discuss, through examples, the basic types of dissipation mechanisms which will be employed in chapters 6 and 8 to model transport in quasiperiodic chains.

\subsection{Single spin $-1 / 2$ coupled to a bath}

In this first example, we will discuss the dynamics of a single spin coupled to a thermal reservoir. Not only this will serve as an illustrative example of the master equation (5.2), but also the same type of baths will be used later in the context of spin chains. The 


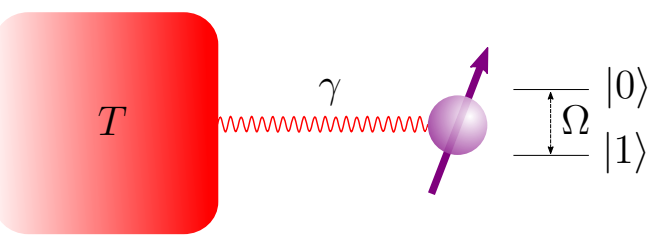

Figure 5.1: Schematic representation of a single spin coupled to a bath at temperature $T$. The constant $\Omega$ is the energy difference between the two energy levels and $\gamma$ is the coupling strength to the bath.

Hamiltonian of the system is given by

$$
H=\frac{\Omega}{2} \sigma^{z},
$$

where $\Omega$ is the energy difference between the two levels. The system is put in contact with a bath with inverse temperature $\beta=1 / T$, as illustrated in Fig. 5.1. The usual master equation used to describe the dynamics in this scenario is given by

$$
\frac{\mathrm{d} \rho}{\mathrm{d} t}=-\frac{i \Omega}{2}\left[\sigma^{z}, \rho\right]+\gamma(1-f) D\left[\sigma^{-}\right](\rho)+\gamma f D\left[\sigma^{+}\right](\rho),
$$

where $D[L]$ are Lindblad dissipators operators of the form (5.4), $\gamma>0$ is the coupling to the bath and $f$ is the Fermi-Dirac distribution, related to the inverse temperature by

$$
f=\frac{1}{e^{\beta \Omega}+1}
$$

The dissipator in Eq. (5.6) is in the Lindblad form [(5.4)] and thus generates a valid CPTP dynamics.

Since the system is $2 \times 2$, the simplest way to solve Eq. 5.6 is to write down the differential equations for each component of the density matrix. The matrix $\rho$ is Hermitian and has trace one, thus it can be parametrized as

$$
\rho=\left(\begin{array}{cc}
p & q \\
q^{*} & 1-p
\end{array}\right)
$$

where $p$ is the population of the excited state $q$ is the coherence. The equations for $p$ and $q$ are obtained by inserting this parametrization in Eq. (5.6) and computing the commutators, which results in 


$$
\begin{aligned}
& \dot{p}=\gamma(f-p) \\
& \dot{q}=-\left(i \Omega+\frac{\gamma}{2}\right) q
\end{aligned}
$$

The solution to these equations are

$$
\begin{aligned}
& p(t)=p(0) e^{-\gamma t}+f\left(1-e^{-\gamma t}\right), \\
& q(t)=e^{-\left(i \Omega+\frac{\gamma}{2}\right)} q(0)
\end{aligned}
$$

In the limit $t \rightarrow \infty$, the system relaxes to its steady-state, given by

$$
\rho^{*}=\lim _{t \rightarrow \infty} \rho=\left(\begin{array}{cc}
f & 0 \\
0 & 1-f
\end{array}\right) .
$$

This corresponds to the equilibrium state,

$$
\rho_{\mathrm{eq}}=\frac{e^{-\beta H}}{Z}=\frac{1}{e^{\beta \Omega}+1}|0\rangle\left\langle 0\left|+\frac{e^{\beta \Omega}}{e^{\beta \Omega}+1}\right| 1\right\rangle\langle 1|
$$

where $Z=\operatorname{Tr}\left\{e^{-\beta H}\right\}$. Therefore, Eq. 5.6 correctly leads the system into a thermal state with inverse temperature $\beta$. The constant $\gamma$ determines the relaxation rate.

The solutions (5.10) shows that the relaxation of the system towards equilibrium can be split into two parts. First, there is the change in populations, from the initial value $p(0)$, to the final value $f$, imposed by the bath. In parallel, however, the system also loses its quantum coherence $q$, which eventually vanishes in equilibrium. The dynamics of $p$ and $q$ are shown in Fig. 5.2.

The master equation (5.6) can be interpreted as a competition between the two terms in the dissipator. The term $D\left[\sigma^{+}\right]$tries to push the spin up, which corresponds to the excited state, whereas $D\left[\sigma^{-}\right]$tries to force the spin down. The final density matrix (5.11) thus reflects a compromise between the two, with the population of the excited sate determined by:

$$
f=\left\langle\sigma^{+} \sigma^{-}\right\rangle_{\mathrm{eq}}
$$

Eq. (5.6) is also frequently introduced in the literature with a different parametrization, given by 

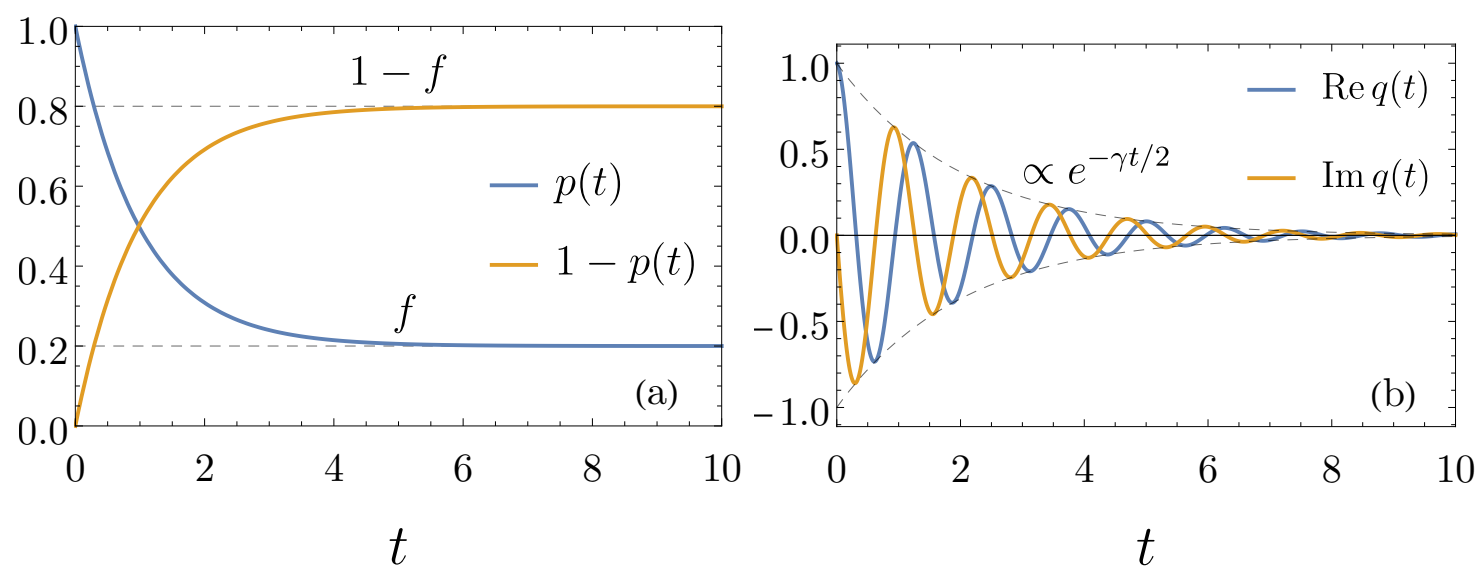

Figure 5.2: (a) Time evolution of the populations $p(t)$ and $1-p(t)$, starting from $p(0)=1$. (b) Time evolution of the real and imaginary parts of the coherence $q(0)$, starting from $q(0)=1$. The parameters used in both plots are $\Omega=5.0, f=0.2$ and $\gamma=1.0$

$$
\mathcal{D}(\rho)=\gamma\left(\frac{1+\mu}{2}\right) D\left[\sigma^{-}\right](\rho)+\gamma\left(\frac{1-\mu}{2}\right) D\left[\sigma^{+}\right](\rho) .
$$

In this case, the steady-state is

$$
\rho^{*}=\left(\frac{1+\mu}{2}\right)|0\rangle\left\langle 0\left|+\left(\frac{1-\mu}{2}\right)\right| 1\right\rangle\langle 1|
$$

thus $\mu$ corresponds to the magnetization in the equilibrium state:

$$
\mu=\left\langle\sigma_{z}\right\rangle_{\mathrm{eq}}
$$

We notice that the two parametrizations are completely equivalent, with the parameters $\mu$ and $f$ related via

$$
f=\frac{1+\mu}{2}
$$

\subsection{Dephasing noise}

We will now consider the same Hamiltonian from Eq. 5.5, but coupled to a different type dissipator, called dephasing noise: 


$$
D(\rho)=\frac{\Gamma}{2} \mathcal{D}\left[\sigma^{z}\right]=\frac{\Gamma}{2}\left(\sigma^{z} \rho \sigma^{z}-\rho\right)
$$

The master equation is then given by

$$
\frac{\mathrm{d} \rho}{\mathrm{d} t}=-\frac{i \Omega}{2}\left[\sigma^{z}, \rho\right]+\frac{\Gamma}{2}\left(\sigma^{z} \rho \sigma^{z}-\rho\right) .
$$

Using the same parametrization for $\rho$ as in the example before, the equations for the components are

$$
\begin{aligned}
& \dot{p}=0 \\
& \dot{q}=-(\Gamma+i \Omega) q .
\end{aligned}
$$

From the first equation, it is possible to see that the dephasing bath does not affect the populations, but only the coherences. The solution for $q$ is given by

$$
q(t)=e^{-(\Gamma+i \Omega) t} q(0)
$$

In the long time limit, the non-diagonal elements vanish

$$
\rho^{*}=\lim _{t \rightarrow \infty} \rho=\left(\begin{array}{cc}
p(0) & 0 \\
0 & 1-p(0)
\end{array}\right)
$$

The dephasing bath preserves the initial populations of the spin, affecting only the offdiagonals.

\subsection{Time evolution of expected values}

In many situations, it may be more useful to compute the expected value of some operator instead looking at the full density matrix. Given some observable $\mathcal{O}$, under the master equation (5.2) its expected value evolves according to

$$
\frac{\mathrm{d}\langle\mathcal{O}\rangle}{\mathrm{d} t}=\operatorname{Tr}\left\{\mathcal{O} \frac{\mathrm{d} \rho}{\mathrm{d} t}\right\}=i\langle[\mathcal{H}, \mathcal{O}]\rangle+\operatorname{Tr}\{\mathcal{O} \mathcal{D}(\rho)\}
$$

where the cyclic property of the trace was used in the unitary term. The dissipative part 
gets a contribution from each of the Lindblad jump operators,

$$
\operatorname{Tr}\{\mathcal{O D}(\rho)\}=\sum_{k} \operatorname{Tr}\left\{\mathcal{O}\left(L_{k} \rho L_{k}^{\dagger}-\frac{1}{2}\left\{L_{k}^{\dagger} L_{k}, \rho\right\}\right)\right\}
$$

Using the cyclic property of the trace, each term in the sum can be written more conveniently as

$$
\operatorname{Tr}\left\{\mathcal{O}\left(L \rho L^{\dagger}-\frac{1}{2}\left\{L^{\dagger} L, \rho\right\}\right)\right\}=\left\langle L^{\dagger} \mathcal{O} L-\frac{1}{2}\left\{L^{\dagger} L, \mathcal{O}\right\}\right\rangle .
$$

The expression in this expected value has a similar structure to the Lindblad dissipators [(5.4)], except thait it acts on an observable $\mathcal{O}$ instead of the density matrix, and the position of $L$ and $L^{\dagger}$ are exchanged in the first term. This motivates the definition of a new superoperator, called the adjoint dissipator, defined as

$$
\bar{D}[L](\mathcal{O})=L^{\dagger} \mathcal{O} L-\frac{1}{2}\left\{L^{\dagger} L, \mathcal{O}\right\}
$$

which can also be written in the more symmetrical form

$$
\bar{D}[L](\mathcal{O})=\frac{1}{2} L^{\dagger}[\mathcal{O}, L]+\frac{1}{2}\left[L^{\dagger}, \mathcal{O}\right] L
$$

Using this definition, the time evolution of $\langle\mathcal{O}\rangle$ can be compactly written as

$$
\frac{\mathrm{d}\langle\mathcal{O}\rangle}{\mathrm{d} t}=i\langle[\mathcal{H}, \mathcal{O}]\rangle+\sum_{k}\left\langle\bar{D}\left[L_{k}\right](\mathcal{O})\right\rangle
$$

\subsection{Single fermionic mode}

To finish this section, we consider now a single fermionic, with the Hamiltonian given by

$$
H=\Omega c^{\dagger} c
$$

When this system is coupled to a bath with inverse temperature $\beta$, the usual master equation is given 


$$
\frac{\mathrm{d} \rho}{\mathrm{d} t}=-i[H, \rho]+\gamma(1-f) D[c](\rho)+\gamma f D\left[c^{\dagger}\right](\rho),
$$

where $f$ is the fermionic occupation number of the bath, given by $f=\left(e^{\beta \Omega}+1\right)^{-1}$. This equation is entirely equivalent to the single spin- $1 / 2$ case [Eq. (5.6)], which in fact is a consequence of the Jordan-Wigner transformation (Appendix A). Notwithstanding, we have found it useful to redo the same calculations in this fermionic language, as this will help gain intuition when we consider a full fermionic chain.

Using Eq. (5.28), the time evolution of $\left\langle c^{\dagger} c\right\rangle$ can be written as

$$
\frac{\mathrm{d}\left\langle c^{\dagger} c\right\rangle}{\mathrm{d} t}=i\left\langle\left[\mathcal{H}, c^{\dagger} c\right]\right\rangle+\gamma(1-f)\left\langle\bar{D}[c]\left(c^{\dagger} c\right)\right\rangle+\left\langle\gamma f \bar{D}\left[c^{\dagger}\right]\left(c^{\dagger} c\right)\right\rangle .
$$

The first adjoint dissipator is given by

$$
\bar{D}[c]\left(c^{\dagger} c\right)=\frac{1}{2} c^{\dagger}\left[c^{\dagger} c, c\right]+\frac{1}{2}\left[c^{\dagger}, c^{\dagger} c\right] c=-c^{\dagger} c
$$

while the second term is

$$
\bar{D}\left[c^{\dagger}\right]\left(c^{\dagger} c\right)=\frac{1}{2} c\left[c^{\dagger} c, c^{\dagger}\right]+\frac{1}{2}\left[c, c^{\dagger} c\right] c^{\dagger}=c c^{\dagger}=1-c^{\dagger} c
$$

where we used the anticommutation rule for fermions, $\left\{c, c^{\dagger}\right\}=1$. Plugging these results together in Eq. (5.31), we obtain

$$
\begin{aligned}
\frac{\mathrm{d}\left\langle c^{\dagger} c\right\rangle}{\mathrm{d} t} & =-\gamma(1-f)\left\langle c^{\dagger} c\right\rangle+\gamma f\left(1-\left\langle c^{\dagger} c\right\rangle\right) \\
& =\gamma\left(f-\left\langle c^{\dagger} c\right\rangle\right)
\end{aligned}
$$

The solution to this equation is given by

$$
\left\langle c^{\dagger} c\right\rangle_{t}=\left\langle c^{\dagger} c\right\rangle_{0} e^{-\gamma t}+f\left(1-e^{-\gamma t}\right)
$$

Thus, the number of fermions in the mode relaxes exponentially to $f$ with a rate $\gamma$. In the spin formulation, $\left\langle c^{\dagger} c\right\rangle$ corresponds to the population of the excited state, $p(t)$, which, by Eq. 5.10, exactly matches this expression. 


\section{Chapter 6}

\section{Boundary-driven quantum chains}

In this chapter, we will describe the boundary-diven quantum chain model, which was the approach we used in this dissertation to study transport in an open-quantum system scenario. In section 6.2, we will compute the time evolution of the covariance matrix, and show it satisfies a Lyapunov equation in the steady-state. Then, in section 6.3, we will discuss the numerical solution to the Lyapunov equation and provide the Mathematica code to do so. Finally, in section 6.4, we will derive an expression for the particle current, the main observable we used to classify the transport regime. The actual results will be discussed in the next chapter.

\subsection{Boundary-driven chains}

In this section, we will finally describe the so-called boundary driven quantum chains, our main model of interest. In order to study transport in an open-quantum system scenario, we considered a quantum chain coupled to reservoirs at each boundary, as illustrated in Fig. 6.1.

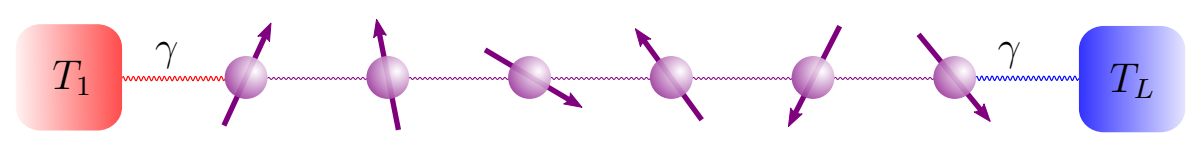

Figure 6.1: Schematic representation of a boundary-driven spin chain.

The Hamiltonian of the chain is the same as in (2.1), which we rewrite here for convenience: 


$$
\mathcal{H}=-\sum_{i=1}^{L-1}\left(c_{i}^{\dagger} c_{i+1}+c_{i+1}^{\dagger} c_{i}\right)+\lambda \sum_{i}^{L} V_{i} c_{i}^{\dagger} c_{i}=\sum_{i j} H_{i j} c_{i}^{\dagger} c_{j}
$$

or, in the spin chain framework,

$$
\mathcal{H}=-\sum_{i=1}^{L-1}\left(\sigma_{i}^{+} \sigma_{i+1}^{-}+\sigma_{i}^{-} \sigma_{i+1}^{+}\right)+\frac{1}{2} \sum_{i}^{L} V_{i} \sigma_{i}^{z}
$$

The effect of the baths can be modeled using different approaches. For example, one might use the non-equilibrium Green's function (NEGF) formalism. In this dissertation, we chose instead to model the system via a Lindblad master equation contaning two dissipators, one for each boundary:

$$
\frac{\mathrm{d} \rho}{\mathrm{d} t}=-i[\mathcal{H}, \rho]+\mathcal{D}_{1}(\rho)+\mathcal{D}_{L}(\rho)
$$

In this approach, the system is called a boundary-driven chain.

There is still a choice regarding the exact form of the dissipators $\mathcal{D}_{i}$. We adopted to work here with the rather straightforward approach of local master equations (LMEs), which consists in using exactly the same dissipator of the single spin case, as in Eq. (5.6), but acting on the sites at the boundaries:

$$
\mathcal{D}_{i}(\rho)=\gamma_{i}\left(1-f_{i}\right) D\left[\sigma_{i}^{+}\right]+\gamma_{i} f_{i} D\left[\sigma_{i}^{-}\right], \quad i=1, L
$$

As in the unitary case, we will work mainly in the fermionic picture, with the following dissipators $^{1}$

$$
\mathcal{D}_{i}(\rho)=\gamma(1-f) D[c]+\gamma f D\left[c^{\dagger}\right], \quad i=1, L
$$

This choice for the dissipators is phenomenological. As we mentioned in the previous chapter, the structure of the jump operators $\left\{L_{i}\right\}$ depend not only on the type of the baths, but also on the system itself. Therefore, the dissipators (6.5) do not model thermal baths, but some different kind of environment, which still generate a valid physical dynamics

\footnotetext{
${ }^{1}$ Under the Jordan-Wigner transformation, the mapping between (6.4) and (6.5) is not exact, since an extra string term is attached. Nonetheless, the time evolution of the CM is exactly the same.
} 
but is simply not thermal in the standard sense. In this case, the parameters $f_{1}$ and $f_{L}$ lack a precise interpretation, as opposed to the single spin case [(5.13)]. For this reason, we shall not try to directly related them with temperature. Instead, we will simply interpret $f_{L}-f_{1}$ as the bias of the boundary drive.

The alternative choice for the dissipators $\mathcal{D}_{i}$ is to use the so-called global master equations (GMEs) [49]. In this approach, the dissipators are obtained from a microscopic derivation, starting from the joint Hamiltonian of the system and bath, and performing a series of approximations to trace out the latter. In Ref. [53], for example, this approach was used for a boundary-driven bosonic chain. The jump operators $\left\{L_{i}\right\}$ obtained in this procedure are non-local, acting on all the sites of chain, despite the fact the baths are coupled only to the boundaries.

\subsection{Lyapunov equation}

In this section, we will study the time evolution of the system under the master equation (6.3). Instead of looking at the full density matrix, which is a $2^{L} \times 2^{L}$ matrix, we characterize the system via the $L \times L$ covariance matrix $(\mathrm{CM})$, defined as

$$
C_{i j}=\left\langle c_{j}^{\dagger} c_{i}\right\rangle
$$

which is a much more easily treatable than the full density matrix. The CM is defined as $\left\langle c_{j}^{\dagger} c_{i}\right\rangle$ rather than $\left\langle c_{i}^{\dagger} c_{j}\right\rangle$ for convenience, as this will later result in cleaner matrix equations. We notice that this definition of the CM is not the most general, since it does not take into account the first moments $\left\langle c_{i}\right\rangle$ and the correlators $\left\langle c_{j}^{\dagger} c_{i}^{\dagger}\right\rangle$. However, for boundary-driven chains these terms vanish in the steady-state, hence the definition (6.6) suffices for our purposes.

The equation of motion of $C$ can be computed from the master equation via Eq. (5.28). We will consider here a slight more general problem, where every site is coupled to a bath, not only the boundaries. By Eq. (5.28), the time evolution of $\left\langle c_{n}^{\dagger} c_{m}\right\rangle$ is

$$
\frac{\mathrm{d}\left\langle c_{n}^{\dagger} c_{m}\right\rangle}{\mathrm{d} t}=i\left\langle\left[\mathcal{H}, c_{n}^{\dagger} c_{m}\right]\right\rangle+\sum_{i=1}^{L} \operatorname{Tr}\left\{c_{n}^{\dagger} c_{m} \mathcal{D}_{i}\right\}
$$


Each term of the sum in the last term is given by

$$
\operatorname{Tr}\left\{c_{n}^{\dagger} c_{m} \mathcal{D}_{i}\right\}=\gamma_{i}\left(1-f_{i}\right) \bar{D}\left[c_{i}\right]\left(c_{n}^{\dagger} c_{m}\right)+\gamma_{i} f_{i} \bar{D}\left[c_{i}^{\dagger}\right]\left(c_{n}^{\dagger} c_{m}\right)
$$

where $\bar{D}_{i}$ has the form (5.27), $f_{n}$ is the Fermi-Dirac distribution of the $n^{\text {th }}$ bath and $\gamma_{n}$ the coupling constant, for $n=1, \ldots, L$. In the computation of the equation of motion the following commutator identities will be useful:

$$
\begin{aligned}
& {\left[c_{i}^{\dagger} c_{j}, c_{n}\right]=-\delta_{i n} c_{j},} \\
& {\left[c_{i}^{\dagger} c_{j}, c_{n}^{\dagger}\right]=\delta_{j n} c_{i}^{\dagger} .}
\end{aligned}
$$

Due to the linearity of the master equation, the coherent and the dissipative contributions to equation of motion of $C$ can be computed separately. The unitary part is given by the first commutator in Eq. (6.7):

$$
\begin{aligned}
{\left[\mathcal{H}, c_{n}^{\dagger} c_{m}\right] } & =\left[\mathcal{H}, c_{n}^{\dagger}\right] c_{m}+c_{n}^{\dagger}\left[\mathcal{H}, c_{m}\right] \\
& =\sum_{i j}\left\{H_{i j}\left[c_{i}^{\dagger} c_{j}, c_{n}^{\dagger}\right] c_{m}+H_{i j} c_{n}^{\dagger}\left[c_{i}^{\dagger} c_{j}, c_{m}\right]\right\} \\
& =\sum_{i j}\left(\delta_{j n} H_{i j} c_{i}^{\dagger} c_{m}-\delta_{i m} H_{i j} c_{n}^{\dagger} c_{j}\right) \\
& =\sum_{i} H_{i n} c_{i}^{\dagger} c_{m}-\sum_{j} H_{m j} c_{n}^{\dagger} c_{j}
\end{aligned}
$$

Notice that, if the system was not coupled to the baths, this would correspond simply to the Heisenberg equations of motion of $c_{n}^{\dagger} c_{m}$. The dissipative contribution to the evolution comes from the adjoint dissipators $\bar{D}\left[c_{i}\right]\left(c_{n}^{\dagger} c_{m}\right)$ and $\bar{D}\left[c_{i}^{\dagger}\right]\left(c_{n}^{\dagger} c_{m}\right)$. The first one is computed as follows:

$$
\begin{aligned}
\bar{D}\left[c_{i}\right]\left(c_{n}^{\dagger} c_{m}\right) & =\frac{1}{2} c_{i}^{\dagger}\left[c_{n}^{\dagger} c_{m}, c_{i}\right]+\frac{1}{2}\left[c_{i}^{\dagger}, c_{n}^{\dagger} c_{m}\right] c_{i} \\
& =-\frac{1}{2} \delta_{i n} c_{i}^{\dagger} c_{m}-\frac{1}{2} \delta_{i m} c_{n}^{\dagger} c_{i} \\
& =-\frac{1}{2} \delta_{i n} c_{n}^{\dagger} c_{m}-\frac{1}{2} \delta_{i m} c_{n}^{\dagger} c_{m} \\
& =-\frac{1}{2}\left(\delta_{i n}+\delta_{i m}\right) c_{n}^{\dagger} c_{m} .
\end{aligned}
$$

From second to the third line, we used the property of the Kronecker delta that $\delta_{i j} F_{j k}=$ 
$\delta_{i j} F_{i k}$, ensuring that the result is proportional to $c_{n}^{\dagger} c_{m}$. The second adjoint dissipator is computed analogously:

$$
\begin{aligned}
\bar{D}\left[c_{i}^{\dagger}\right]\left(c_{n}^{\dagger} c_{m}\right) & =\frac{1}{2} c_{i}\left[c_{n}^{\dagger} c_{m}, c_{i}^{\dagger}\right]+\frac{1}{2}\left[c_{i}, c_{n}^{\dagger} c_{m}\right] c_{i}^{\dagger} \\
& =\frac{1}{2} \delta_{i m} c_{i} c_{n}^{\dagger}+\frac{1}{2} \delta_{i n} c_{m} c_{i}^{\dagger} \\
& =\frac{1}{2} \delta_{i m} c_{m} c_{n}^{\dagger}+\frac{1}{2} \delta_{i n} c_{m} c_{n}^{\dagger} \\
& =\frac{1}{2}\left(\delta_{i n}+\delta_{i m}\right) c_{m} c_{n}^{\dagger} .
\end{aligned}
$$

Plugging these two results in Eq. (6.8) yields

$$
\begin{aligned}
\operatorname{Tr}\left\{c_{n}^{\dagger} c_{m} \mathcal{D}_{i}\right\} & =\gamma_{i}\left(1-f_{i}\right)\left[-\frac{1}{2}\left(\delta_{i n}+\delta_{i m}\right) c_{n}^{\dagger} c_{m}\right]+\gamma_{i} f_{i}\left[\frac{1}{2}\left(\delta_{i n}+\delta_{i m}\right) c_{m} c_{n}^{\dagger}\right] \\
& =-\frac{\gamma_{i}}{2} \delta_{i n} c_{i}^{\dagger} c_{m}-\frac{\gamma_{i}}{2} \delta_{i m} c_{n}^{\dagger} c_{i}+\gamma_{i} f_{i} \delta_{i n} \delta_{n m} .
\end{aligned}
$$

Finally, combining the unitary and dissipative part in Eq. (6.7) we get

$$
\frac{\mathrm{d}\left\langle c_{n}^{\dagger} c_{m}\right\rangle}{\mathrm{d} t}=-\sum_{i}\left(-i H_{i n}+\frac{\gamma_{i}}{2} \delta_{i n}\right)\left\langle c_{i}^{\dagger} c_{m}\right\rangle-\sum_{j}\left(i H_{m j}+\frac{\gamma_{j}}{2} \delta_{j m}\right)\left\langle c_{n}^{\dagger} c_{j}\right\rangle+\gamma_{n} f_{n} \delta_{n m},
$$

Using the definition of the covariance matrix, this equation can be written compactly in matrix notation as

$$
\frac{\mathrm{d} C}{\mathrm{~d} t}=-\left(W C+C W^{\dagger}\right)+F
$$

where the matrix $W$ is defined as

$$
W=i H+\frac{\Gamma}{2}
$$

with $\Gamma=\operatorname{diag}\left(\gamma_{1}, \gamma_{2}, \ldots, \gamma_{L}\right)$ and $F=\operatorname{diag}\left(\gamma_{1} f_{1}, \gamma_{2} f_{2}, \ldots, \gamma_{L} f_{L}\right)$. In the NESS, $\mathrm{d} C / \mathrm{d} t=$ 0 , therefore the CM satisfies

$$
W C+C W^{\dagger}=F
$$


which is known as the Lyapunov Equation.

It is worth mentioning that the system described by the master equation (6.3) is gaussian, because all terms are at most quadratic in the fermionic operators. When gaussianity holds, the covariance matrix contains all information about the system, and the density matrix can be written in terms of its components. Despite this fact, we did not have to make explicit use of gaussianity, since all our observables of interest are already elements of the CM.

\subsection{Numerical computation of the NESS}

The Lyapunov equation (6.17) is ubiquitous in control theory, also appearing in a wide range of contexts. As such, most numerical linear algebra packages provide off-the-shelf solvers to this equation, usually based in the Bartles-Stewart algorithm [54], whose computational complexity is $\mathcal{O}\left(L^{3}\right)$. In Mathematica, for example, Eq. (6.17) can be solved using the function LyapunovSolve. Alternatively, the solution can be found using the eigendecomposition of the non-hermitian matrix $W$, as described in [17]. We have found that this second procedure is faster for our particular system. In this section, we will discuss this method and provide the explicit code for the solution, written in Mathematica.

The formal solution to Eq. (6.15) is given by

$$
C(t)=e^{-W t} C(0) e^{-W^{\dagger} t}+\int_{0}^{t} \mathrm{~d} t^{\prime} e^{-W\left(t-t^{\prime}\right)} F e^{-W^{\dagger}\left(t-t^{\prime}\right)} .
$$

Making the substitution $t-t^{\prime} \rightarrow t^{\prime}$ and taking the limit $t \rightarrow \infty$, we arrive at an expresion for the steady-state,

$$
C=\int_{0}^{\infty} \mathrm{d} t e^{-W t} F e^{-W^{\dagger} t}
$$

where we write $C=C(\infty)$ for simplicity. This is the formal solution to the Lyapunov equation [Eq. (6.17)]. Although the matrix $W$ is not hermitian it is diagonalizable, and thus can be decomposed as 


$$
W=S \Lambda S^{-1},
$$

where $\Lambda=\operatorname{diag}\left(\lambda_{1}, \ldots, \lambda_{L}\right)$. Using this decomposition, the matrix exponentials can be written as $e^{-W t}=S e^{-\Lambda t} S^{-1}$ and $e^{-W^{\dagger} t}=\left(S^{-1}\right)^{\dagger} e^{-\Lambda^{*} t} S^{\dagger}$. Inserting this expression in Eq. (6.19), one obtains

$$
C=S\left[\int_{0}^{\infty} \mathrm{d} t e^{-\lambda t} S^{-1} F\left(S^{-1}\right)^{\dagger} e^{-\lambda^{*} t}\right] S^{\dagger}
$$

Therefore, the covariance can be written as

$$
C=S Y S^{\dagger}
$$

where $Y$ is the matrix in brackets in Eq. (6.21), whose elements are

$$
\begin{aligned}
Y_{i j} & =\sum_{k}\left(S^{-1}\right)_{i k}\left(S^{-1}\right)_{j k}^{*} \gamma_{k} f_{k} \int_{0}^{\infty} \mathrm{d} t e^{-\left(\lambda_{i}+\lambda_{j}^{*}\right) t} \\
& =\sum_{k}\left(S^{-1}\right)_{i k}\left(S^{-1}\right)_{j k}^{*} \frac{\gamma_{k} f_{k}}{\lambda_{i}+\lambda_{j}^{*}} .
\end{aligned}
$$

In our numerical simulations, we considered only the boundary-driven case where $F=\operatorname{diag}\left(\gamma f_{1}, 0, \ldots, 0, \gamma f_{L}\right)$, but the code can be easily generalized to accomodate couplings to every site. The following Mathematica functions were used to compute the NESS: 


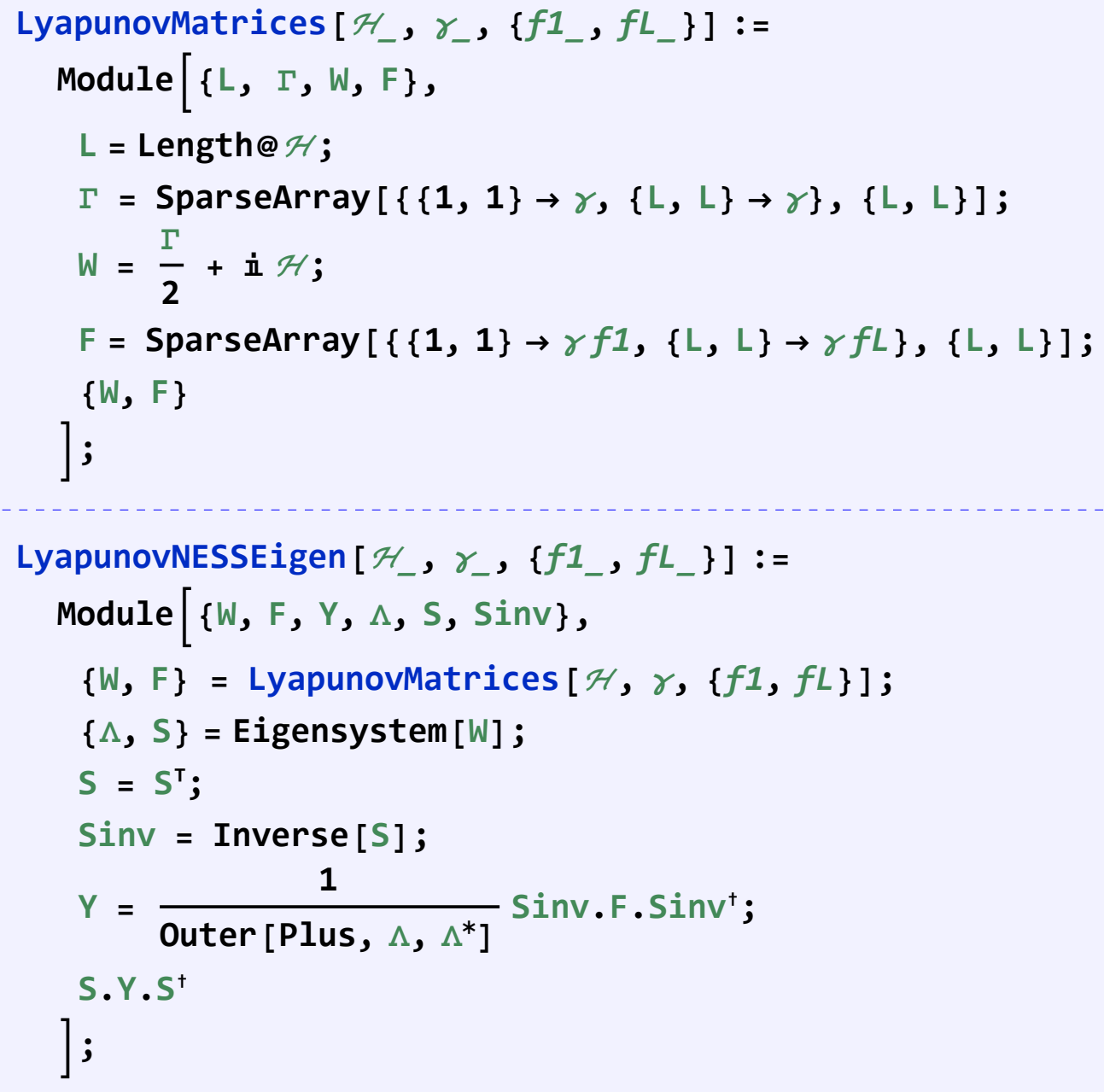

The function LyapunovMatrices returns the matrices $W$ and $F$ and the function LyapunovNESSEigen computes the covariance matrix in the NESS.

If one wishes, instead, to use Mathematica's built-in implementation, then the function LyapunovNESSEigen can instead be replaced by

LyapunovNESS $\left[\mathcal{H}_{-}, \gamma_{-},\left\{f 1_{-}, f L_{-}\right\}\right]:=$

Module $[\{W, F\}$,

$\{\mathrm{W}, \mathrm{F}\}=$ LyapunovMatrices $[\mathcal{H}, \gamma,\{f 1, f L\}]$;

LyapunovSolve [W, F]

] ; 


\subsection{Particle/spin current operator}

The main observable of interest in the classification of the transport regime is the particle current, which we now define. We start be looking at the time evolution of the mean occupation number for some site in the middle of the chain, given by $\left\langle n_{i}\right\rangle=\left\langle c_{i}^{\dagger} c_{i}\right\rangle$, for $1<i<L$. According to Eq. (6.14), this evolution is given by

$$
\frac{\mathrm{d}\left\langle n_{i}\right\rangle}{\mathrm{d} t}=i\left\langle c_{i}^{\dagger} c_{i-1}-c_{i-1}^{\dagger} c_{i}\right\rangle-i\left\langle c_{i+1}^{\dagger} c_{i}-c_{i}^{\dagger} c_{i+1}\right\rangle, \quad 1<i<L
$$

This expression can be interpreted as a continuity equation,

$$
\frac{\mathrm{d}\left\langle n_{i}\right\rangle}{\mathrm{d} t}=\left\langle J_{i-1}\right\rangle-\left\langle J_{i}\right\rangle
$$

where $J_{i}$ is the particle current operator at site $i$, defined as

$$
J_{i}=i\left(c_{i+1}^{\dagger} c_{i}-c_{i}^{\dagger} c_{i+1}\right)
$$

The term $\left\langle J_{i}\right\rangle$ thus corresponds the particle current entering the mode $i$, while $\left\langle J_{i}\right\rangle$ accounts for the current leaving mode $i$ towards mode $i+1$.

The time evolution of $\left\langle n_{i}\right\rangle$ at the boundaries are slightly different from Eq. 6.24, but can still be interpreted as a continuity equation. At the left boundary, one has

$$
\begin{aligned}
\frac{\mathrm{d}\left\langle n_{1}\right\rangle}{\mathrm{d} t} & =\gamma\left(f_{1}-\left\langle c_{1}^{\dagger} c_{1}\right\rangle\right)-i\left\langle c_{2}^{\dagger} c_{1}-c_{1}^{\dagger} c_{2}\right\rangle \\
& =\left\langle J_{\mathcal{D}_{1}}\right\rangle-\left\langle J_{1}\right\rangle,
\end{aligned}
$$

where we defined

$$
J_{\mathcal{D}_{1}}=\gamma\left(f_{1}-\left\langle c_{1}^{\dagger} c_{1}\right\rangle\right)
$$

corresponding to the current of quanta coming from the left bath to the system. Similarly, for the last site one may write

$$
\frac{\mathrm{d}\left\langle n_{L}\right\rangle}{\mathrm{d} t}=\left\langle J_{L}\right\rangle-\left\langle J_{\mathcal{D}_{L}}\right\rangle .
$$


where $\left\langle J_{\mathcal{D}_{L}}\right\rangle=\gamma\left(f_{L}-\left\langle c_{L}^{\dagger} c_{L}\right\rangle\right)$ is the current leaving the last site into the right bath.

In the NESS, $\mathrm{d}\left\langle n_{i}\right\rangle / \mathrm{d} t=0$, and so all currents must coincide:

$$
\left\langle J_{\mathcal{D}_{1}}\right\rangle=\left\langle J_{1}\right\rangle=\cdots=\left\langle J_{L}\right\rangle=\left\langle J_{\mathcal{D}_{L}}\right\rangle \equiv J
$$

Therefore, we may unequivocally refer to the current flowing through the chain simply as $J$. In terms of the covariance matrix, we have that

$$
J=i\left(C_{i, i+1}-C_{i+1, i}\right)
$$

Using the fact that $C_{i+1, i}=-C_{i, i+1}^{*}$, this equation may be written conveniently as

$$
J=2 \operatorname{Im} C_{i, i+1}
$$

which is the expression we adopted in the numerical simulations.

In the spin chain formulation, the particle current is naturally translated into a spin current. Using the Jordan-Wigner transformation (Appendix A), one may show that

$$
J=2 i\left\langle\sigma_{i}^{x} \sigma_{i+1}^{y}-\sigma_{i}^{y} \sigma_{i+1}^{x}\right\rangle
$$

The same expression may be obtained by writing an explicit expression for $\mathrm{d}\left\langle\sigma_{i}^{z}\right\rangle / \mathrm{d} t$ and interpreting it as a continuity equation, in a similar spirit to Eq. (6.24). 


\section{Chapter 7}

\section{Non-equilibrium transport properties}

\subsection{Classification of the transport properties via the par- ticle current}

As discussed in the introduction, when a metal bar is put in contact with two reservoirs at its boundary, the heat current in the NESS is inversely proportional to its length:

$$
J=\kappa \frac{\Delta T}{L} \propto \frac{1}{L}
$$

which characterizes a typical diffusive scaling. In boundary-driven quantum chains, however, the situation is different. In a non-interacting chain with no on-site potential, the particle in the NESS exhibits a ballistic behavior, as we will discuss in section. Moreover, with the addition of quasiperiodic potentials, a wide range of transport regimes may emerge. In general, the particle current in the NESS scales as

$$
J \sim \frac{1}{L^{\nu}}
$$

where $\nu$ is a non-negative constant which we refer to as non-equilibrium transport coefficient, and $J$ is defined in Eq. (6.32).

As in the coherent dynamics scenario, the coefficient $\nu$ can be used to classify the transport regimes of the system. If $\nu=0$, the transport is ballistic, and $J$ is independent of the system size, and $\nu=1$ corresponds to diffusive scaling. Other values of $\nu$ are 
considered anomalous transport, which is further classified as superdiffusive if $0<\nu<1$ or subdiffusive if $\nu>1$. The absence of transport, which happens for instance in localized systems, can be seen as an extreme case of subdiffusion where $\nu \rightarrow \infty$. A summary of these classifications is given in Table 7.1.

Table 7.1: Classification of the non-equilibrium transport regime using the value of $\nu$.

\begin{tabular}{cc}
\hline Transport regime & Transport coefficient \\
\hline Ballistic & $\nu=0$ \\
Superdiffusive & $0<\nu<1$ \\
Diffusive & $\nu=1$ \\
Subdiffusive & $\nu>1$ \\
Localized & $\nu=\infty$ \\
\hline
\end{tabular}

The procedure to compute the coefficient $\nu$ is illustrated in Fig. 7.1. The exact value of the coefficient depends on the numerical theoretical properties of the chosen family of sizes $L$. As usual in the literature, we chose the sizes $L$ to be Fibonacci numbers, which are known to yield smooth curves for the current for the quasiperiodic potentials. The value of $\nu$ are then computed by fitting a linear regression of the following form to the data points:

$$
\log J=\nu \log L+C
$$

In order to approximate the value of the current in the thermodynamic limit, the linear regression is performed using only the five largest values of $L$ we are able to simulate, which are highlighted in blue in Fig. 7.1.

\subsection{Connection between transport coefficients}

The coefficients $\alpha$ and $\nu$ can be related via a simple relation, provided that one assumes that the scalings in the system are governed by a single exponent in both the unitary and non-equilibrium scenarios. In closed system, the width of the wave function follows the scaling $\sqrt{\left\langle(\Delta x)^{2}\right\rangle} \sim t^{\alpha}$, thus the characteristic time it takes for a particle to cross the whole chain is $\tau \sim L^{1 / \alpha}$. In the NESS, the particle current should equal the rate of 


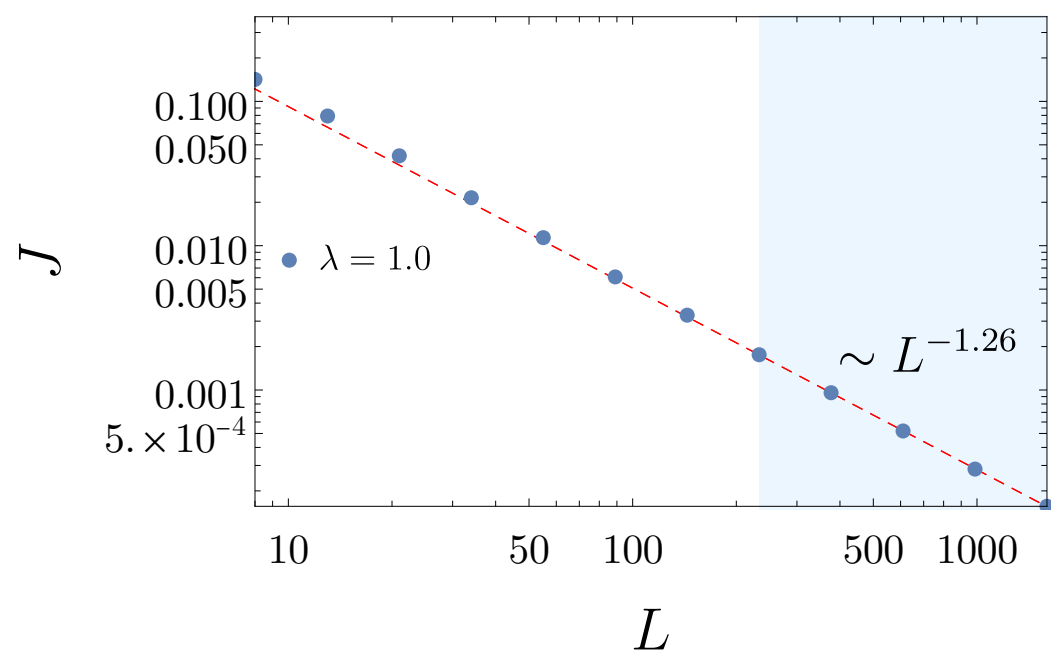

Figure 7.1: Illustration of the procedure used to computed the transport coefficient $\nu$ in Eq. (7.2). Only the last five points are used in the regression. This particular example corresponds to the critical point of the AAH, averaged over 100 values of $\theta$, which is subdiffusive.

particles flowing through the system,

$$
J \sim \frac{L}{\tau} \sim \frac{L}{L^{1 / \alpha}}
$$

Comparing this expression with the scaling $J \sim L^{-\nu}$, we obtain the relation

$$
\alpha=\frac{1}{1+\nu}
$$

Notice, however, that the hypothesis that the transport is governed by a single exponent is central to this derivation. Although this may be true in some cases, and indeed Eq. (7.5) has been explicitly verified in some classical [55] and quantum systems, it is still somewhat arbitrary, and there is no a priori reason it should hold.

In fact, as discussed, in Ref. [17], this hypothesis breaks down in the Fibonacci model and in the critical point of the AAH model, and hence Eq. (7.5) is not satisfied.

Nonetheless, we will still make use Eq. (7.5) in chapter 8, where we discuss the effect of dephasing on the system, which will make possible to explain some qualitative behaviors of the particle current. In this case, Eq. (7.5) may be understood as the definition of some "effective transport coefficient" of excitations moving trough the chain in the NESS. 


\subsection{Transport with zero potential}

When the on-site potential is constant, the covariance matrix in the NESS can be found analytically. In this section, we will briefly describe the approach used in [8]. In this work, they considered a boundary-driven bosonic chain, but all the equations have exactly the same structure to the fermionic case.

When there is no bias between the baths, that is, $f_{1}=f_{L}=f$, the solution to the Lyapunov Equation (6.17) is $C=f \mathbb{1}$, which can be readily verified by substitution:

$$
\begin{aligned}
f W \mathbb{1}+f \mathbb{1} W^{\dagger} & =f\left(i H+\frac{\Gamma}{2}\right)+f\left(-i H+\frac{\Gamma}{2}\right) \\
& =f \Gamma \\
& =F .
\end{aligned}
$$

Therefore, when there is no bias, the final population of the excited state in every site will the same, given by the value $f$ determined by the bath. Interestingly, in this case all the sites correctly thermalize independently of each other, even with our choice of local dissipators. This fact is the motivates the following ansatz to the general case:

$$
C=\bar{f} \mathbb{1}+\Delta f D
$$

where

$$
\bar{f}=\frac{f_{1}+f_{L}}{2} \text { and } \quad \Delta f=\frac{f_{1}-f_{L}}{2}
$$

and $D$ is a new matrix, to be determined from the Lyapunov equation. The first term captures the equilibrium state to which the system would converge if $f_{1}$ and $f_{L}$ substituted by their average, while the second term is a perturbation caused the bias of the baths. In particular, the particle current is determined by the coherences of $D$, that is, $J=$ $\Delta f \operatorname{Im} D_{i, i+1}$.

Using the definition of $W[(6.16)]$, the Lyapunov equation can be rewritten as

$$
i[H, C]+\frac{1}{2}\{C, \Gamma\}=F .
$$


Substituting the ansatz (7.8), one obtains

$$
i[H, D]+\frac{1}{2}\{D, \Gamma\}=\tilde{\Gamma},
$$

where

$$
\tilde{\Gamma}=\frac{1}{\Delta f}(F-f \Gamma)=\operatorname{diag}\left(\gamma_{1}, 0, \ldots, 0,-\gamma_{L}\right)
$$

The solution to this system, assuming $\gamma_{1}=\gamma_{L}=\gamma$, has a simple tridiagonal structure, as discussed in [8], given by

$$
D=\frac{2 \gamma}{4+\gamma^{2}}\left(\begin{array}{ccccc}
\gamma & -i & & & \\
i & 0 & -i & & \\
& i & \ddots & \ddots & \\
& & \ddots & 0 & -i \\
& & & i & -\gamma
\end{array}\right)
$$

Therefore, the particle current in the NESS is

$$
J=\frac{2 \gamma}{4+\gamma^{2}}\left(f_{1}-f_{L}\right) \sim L^{0}
$$

This expression is independent of the system size, which characterizes ballistic transport. Moreover, the mean occupation number is equal to $\bar{f}$ for all the sites inside the chain, whereas in the border they are

$$
\left\langle n_{1}\right\rangle=\bar{f}+\frac{2 \gamma^{2}}{4+\gamma^{2}} \Delta f \quad \text { and } \quad\left\langle n_{L}\right\rangle=\bar{f}-\frac{2 \gamma^{2}}{4+\gamma^{2}} \Delta f
$$

Therefore, the baths simply shift the occupation number of the boundary sites in opposite directions, while the occupation number is constant inside the chain. This is also a typical signature of ballistic behavior, as opposed to what happens, for instance, in a macroscopic metal bar coupled to baths, whose temperature profile in the NESS is linear. 

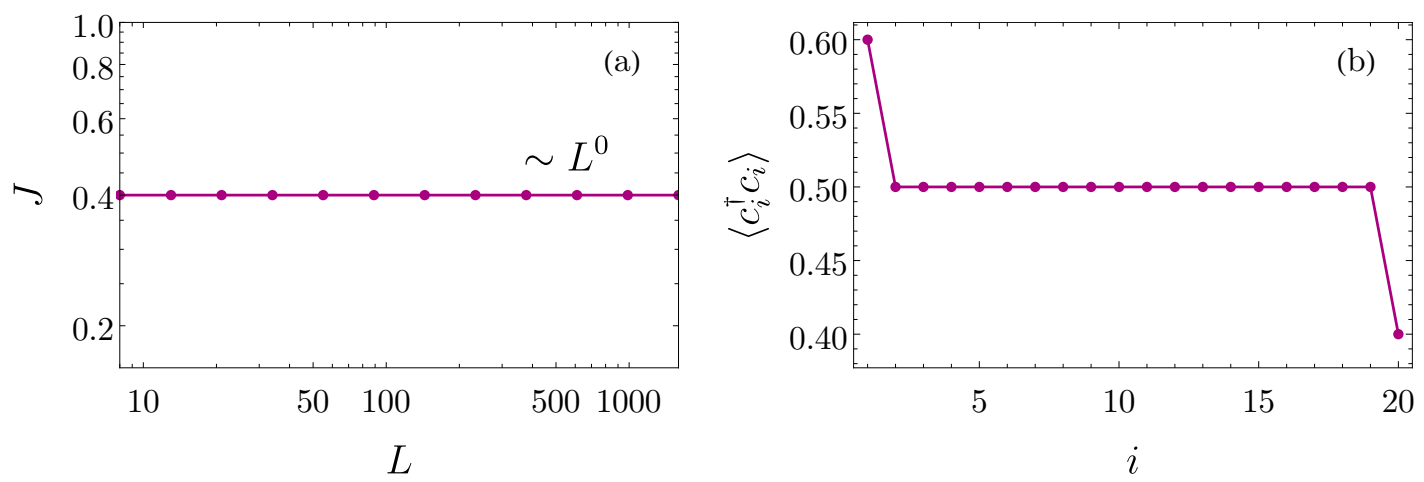

Figure 7.2: (a) Scaling of the current with respect to $L$ with zero on-site potential. The current is independent of the system size, thus the transport is ballistic. (b) Population profile in the NESS for a chain with $L=20$. In both plots, the other parameters are $\gamma=1, f_{1}=1$ and $f_{L}=0$.

\subsection{Transport properties with quasiperiodic potentials}

In this section, we will discuss the transport properties of the boundary-driven AAH and Fibonacci models. The non-equilibrium transport properties of the AAH model are summarized in Fig. 7.3, which shows the scaling of the particle current with $L$ for different values of $\lambda$. As in the unitary case, the presence of a localization transition is clear. For $\lambda<1$, which corresponds to the extended phased, the transport is ballistic, as one would expect. On the other hand, when $\lambda>1$, which corresponds to localized eigenstates, the current decays exponentially with the system size. This shows that the current vanishes in the thermodynamic limit, although it is positive for a finite system. We estimate the rate of decay by fitting a curve of the form $L \sim e^{-\delta L}$ to the simulation data.

At the critical point $\lambda=1.0$, when eigenstates are neither localized nor delocalized, the transport is subdiffusive. The exact value of $\alpha$ depends the phase $\theta$ and on the family of sizes one chooses to perform the linear regression [17]. In the particular case of Fig. 7.3, the current is averaged over $\theta$ and the system sizes are Fibonacci numbers, which results in the value $\nu=1.26$. This coefficient is very close to the result report in Ref. [17], which is 1.27 .

This result shows that the connection between the coefficients [(7.5)] breaks down in the critical point. The value $\nu=1.26$ corresponds to an effective unitary coefficient of approximately $1 /(1+1.26) \approx 0.44$, but, as shown in Fig. 4.4 , the unitary transport is diffusive at $\lambda=1.0$, with $\alpha=0.5$. 
Interestingly, the connection is indeed satisfied for the symmetrical version of the potential. In Ref. [17], it is shown that, in the critical point of the symmetric version, the current scales as $J \sim L^{-1.1}$. Therefore, the effective coefficient is $1 /(1+1.1) \approx 0.476$, which is remarkably close to the value of $\alpha$, as shown in Fig. 4.4.

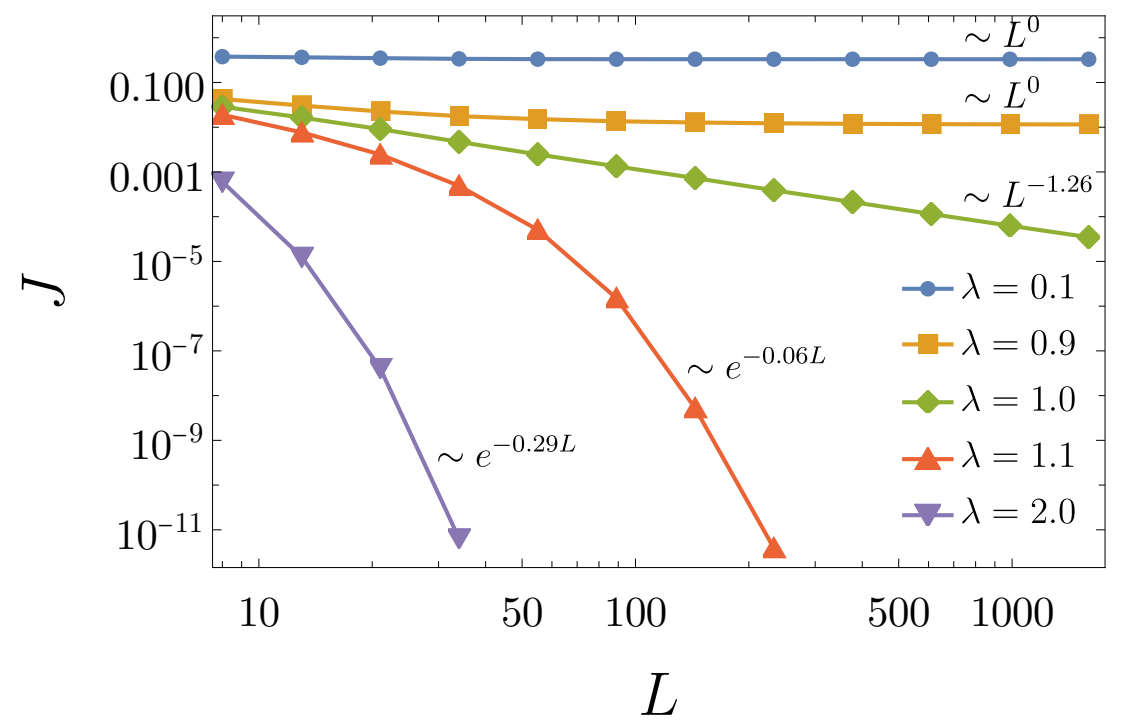

Figure 7.3: Scaling of the current with system size for the AAH model for different values of $\lambda$. For $\lambda<1$, the transport is ballistic. For $\lambda=1$, the transport is subdiffusive, with $J \sim L^{-1.26}$. For $\lambda>1$, the current decays exponentially with $L$. The linear regression is performed using the last five points for $\lambda \leq 1$ and all available the points for $\lambda>1$. The current is averaged over 100 values of $\theta$ evenly spaced in between 0 and $\pi$. The system sizes are Fibonacci numbers.

The analogous curves for the Fibonacci model are shown in Fig. 7.4. Similarly to the unitary the case, the system can be tuned to exhibit any type of transport behavior. Notice that whenever the value of $\lambda$ is increased the slope of the curve decreases. For values of $\lambda$ smaller the 3 , the system is superdiffusive. For $\lambda=3.0$, the transport is very close the diffusive. For values of $\lambda$ above this value, the system is subdiffusive.

The dependence of the coefficient $\nu$ is shown in Fig. 7.5. As in the unitary case, the system goes continuously from the ballistic regime to subdiffusion when $\lambda$ is increased. The diffusive regime, indicated by the dashed line, occurs for $\lambda \approx 3$.

As we mentioned, in the Fibonacci model the connection between the exponents $\alpha$ and $\nu$ is also broken. For instance, when $\lambda=2.0$, we found that $\nu=0.69$, which corresponds to an effective unitary coefficient of $\approx 0.63$, which slightly differs from the actual value 0.61 . 


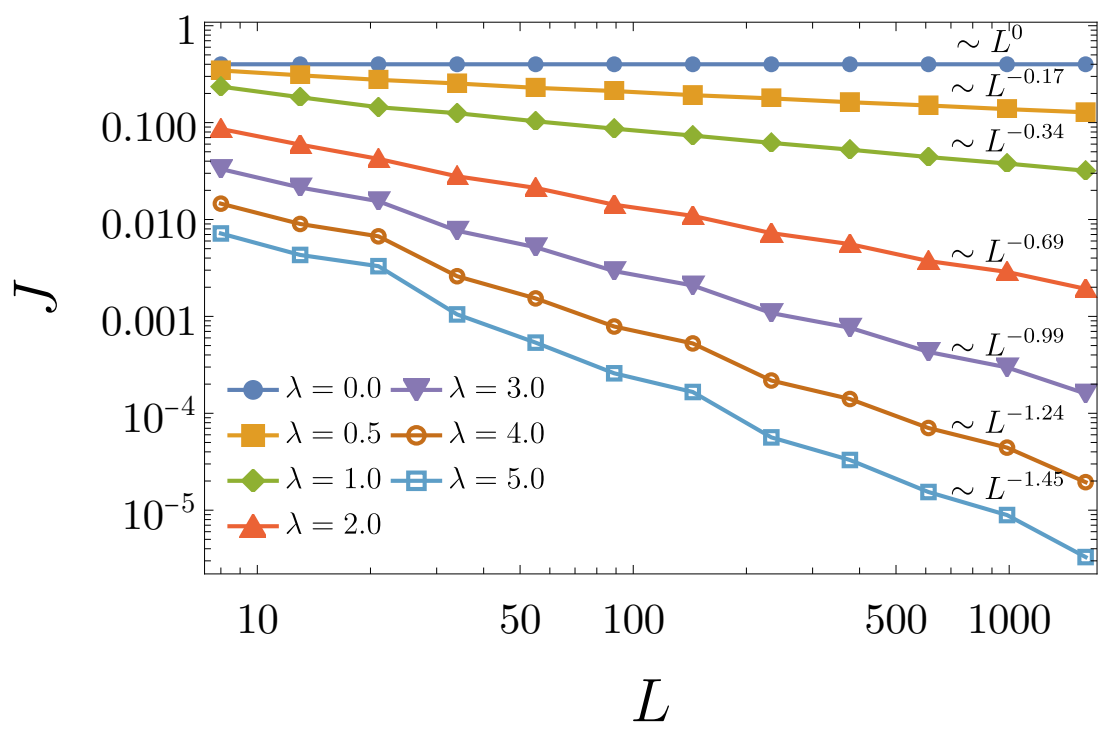

Figure 7.4: Scaling of the current with system size in the Fibonacci model for different values of $\lambda$. The last five points of each curve were used to compute the coefficient $\nu$. The system sizes were chosen as Fibonacci numbers.

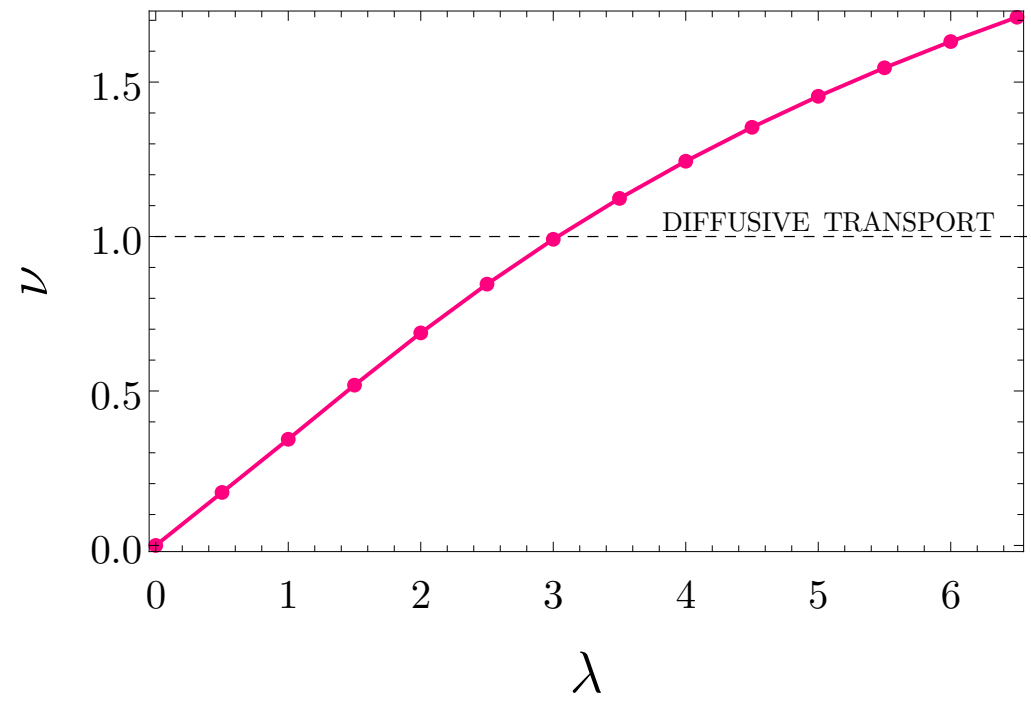

Figure 7.5: Dependence of the exponent $\nu$ with the system size $L$ in the Fibonacci model. The exponent was computed by fitting a power-law $J \sim L^{-\nu}$ to the simulation data, using the last five points for each value of $\lambda$. 


\section{Chapter 8}

\section{Dephasing enhanced transport}

In this final chapter we discuss an original contribution from this work, which aims to understand the competition between quasiperiodicity and dephasing in one-dimensional chains.

\subsection{Boundary-driven chains with dephasing}

In the presence of dephasing, the master equation is modified to

$$
\frac{\mathrm{d} \rho}{\mathrm{d} t}=-i[\mathcal{H}, \rho]+\mathcal{D}_{1}(\rho)+\mathcal{D}_{L}(\rho)+\sum_{i=1}^{L} \mathcal{D}_{i}^{\mathrm{deph}}(\rho)
$$

where the dissipator $\mathcal{D}_{i}^{\text {deph }}$ models the effect of dephasing coupled to site $i$. It has the same form as in Eq. (5.18),

$$
\mathcal{D}_{i}^{\text {deph }}(\rho)=\frac{\Gamma}{2} D\left[\sigma_{i}^{z}\right]=\frac{\Gamma}{2}\left(\sigma_{i}^{z} \rho \sigma_{i}^{z}-\rho\right),
$$

or, using the Jordan-Wigner transformation,

$$
\mathcal{D}_{i}^{\mathrm{deph}}(\rho)=\Gamma D\left[c_{i}^{\dagger} c_{i}\right]=\Gamma\left(c_{i}^{\dagger} c_{i} \rho c_{i}^{\dagger} c_{i}-\frac{1}{2}\left\{\left(c_{i}^{\dagger} c_{i}\right)^{2}, \rho\right\}\right)
$$

In this case, the mapping is exact, since $\sigma_{i}^{z}=2 c_{i}^{\dagger} c_{i}-1$, for any $i$.

Notice that the dissipators modeling the dephasing noise are quartic in the fermionic 
operators. Therefore, when they are included in the master equation, it will no longer be gaussian preserving. Notwithstanding, it turns out that the equations for the covariance matrix remain closed. This only happens because the tight-binding chain with dephasing has the nice property that the equations for the second moments are uncoupled from the higher-order moments $[7,8,56]$.

The time evolution of the covariance matrix can again be computed using Eq. 5.28. Because of the linearity of the master equation, the dephasing dissipators simply add an extra term to Eq. 6.14, namely

$$
\sum_{i} \bar{D}\left[n_{i}\right]\left(c_{n}^{\dagger} c_{m}\right)
$$

Each of the terms in the sum can be computed in a similar fashion to what was done in section 6.2. To this end, the following commutator will be useful:

$$
\left[n_{i}, c_{n}^{\dagger} c_{m}\right]=\left[c_{i}^{\dagger} c_{i}, c_{n}^{\dagger}\right] c_{m}+c_{n}^{\dagger}\left[c_{i}^{\dagger} c_{i}, c_{m}\right]=\left(\delta_{i n}-\delta_{i m}\right) c_{n}^{\dagger} c_{m}
$$

Thus, using the expression for the adjoint dissipation given in Eq. (5.27), one obtains

$$
\begin{aligned}
\bar{D}\left[n_{i}\right]\left(c_{n}^{\dagger} c_{m}\right) & =\frac{1}{2} n_{i}\left[c_{n}^{\dagger} c_{m}, n_{i}\right]+\frac{1}{2}\left[n_{i}, c_{n}^{\dagger} c_{m}\right] n_{i} \\
& =\frac{1}{2}\left(\delta_{i m}-\delta_{i n}\right) n_{i} c_{n}^{\dagger} c_{m}-\frac{1}{2}\left(\delta_{i m}-\delta_{i n}\right) c_{n}^{\dagger} c_{m} n_{i} \\
& =\frac{1}{2}\left(\delta_{i m}-\delta_{i n}\right)\left[n_{i}, c_{n}^{\dagger} c_{m}\right] \\
& =\frac{1}{2}\left(\delta_{i m}+\delta_{i n}-2 \delta_{i m} \delta_{i n}\right) c_{n}^{\dagger} c_{m}
\end{aligned}
$$

where in the last step we used the fact that $\delta_{i j}^{2}=\delta_{i j}$ to write $\left(\delta_{i n}-\delta_{i m}\right)^{2}=\left(\delta_{i m}+\delta_{i n}-\right.$ $\left.2 \delta_{i m} \delta_{i n}\right)$. The extra term added by the dephasing dissipators is then

$$
\sum_{i} \bar{D}\left[n_{i}\right]\left(c_{n}^{\dagger} c_{m}\right)=\left(1-\delta_{n m}\right) c_{n}^{\dagger} c_{m}
$$

Therefore, the time evolution of $\left\langle c_{n}^{\dagger} c_{m}\right\rangle$ is modified to

$$
\frac{\mathrm{d}\left\langle c_{n}^{\dagger} c_{m}\right\rangle}{\mathrm{d} t}=-\sum_{i} W_{m i}\left\langle c_{n}^{\dagger} c_{i}\right\rangle-\sum_{i} W_{n i}^{*}\left\langle c_{i}^{\dagger} c_{m}\right\rangle-\Gamma\left(1-\delta_{n m}\right) c_{n}^{\dagger} c_{m}+\gamma_{n} f_{n} \delta_{n m}
$$


In terms of the covariance matrix, this equation can be written more neatly in matrix notation as

$$
\frac{\mathrm{d} C}{\mathrm{~d} t}=-\left(W C+C W^{\dagger}\right)-\Gamma \Delta(C)+F
$$

where $\Delta(\cdot)$ is an operation the removes the diagonal of a matrix:

$$
\Delta(C)=C-\operatorname{diag}\left(C_{11}, \ldots, C_{L L}\right)
$$

Moreover, the quantity $\Gamma$ multiplying $\Delta(C)$ refers to the constant appearing in the dephasing dissipator (8.2) and not the matrix $\Gamma$ used in the previous chapter. We apologize for the confusion in the notation.

In the NESS, $\mathrm{d} C / \mathrm{d} t=0$, thus the covariance matrix satisfies

$$
W C+C W^{\dagger}+\Gamma \Delta(C)=F
$$

This equation is not in Lyapunov form, thus it can't be solved using specialized routines or the method we described in section 6.3. Nevertheless, it is still a sparse linear system in the entries of the matrix $C$, and can be solved using standard solvers built-in in any linear algebra package. We now proceed to describe how to accomplish this.

\subsection{Non-equilibrium steady state}

In this section we will now describe how Eq. (8.11) can be cast into a more familiar linear system notation, by defining the vectorization operation. The vec operation converts a matrix into a vector by stacking all columns, as in this $2 \times 2$ example:

$$
\operatorname{vec}\left(\begin{array}{ll}
a & b \\
c & d
\end{array}\right)=\left(\begin{array}{l}
a \\
c \\
b \\
d
\end{array}\right)
$$

More generally, the operation vec maps an $L \times L$ matrix into a vector with $L^{2}$ elements. The vectorization operation has many useful properties. For example, the vectorization of a product of three matrices is given by 


$$
\operatorname{vec}(A B C)=\left(C^{\top} \otimes A\right) \operatorname{vec}(B)
$$

The vectorization of a product $A B$ is a special case of this property, in which $C=\mathbb{1}$ :

$$
\operatorname{vec}(A B)=\operatorname{vec}(A B \mathbb{1})=(\mathbb{1} \otimes A) \operatorname{vec}(B)
$$

This expression can be used to vectorize all the terms in Eq. (8.11). Suppose at first that $\Gamma=0$, which means that $C$ satisfies the Lyapunov equation (6.17). Using the vectorization operation, one obtains

$$
\left(\mathbb{1} \otimes W+W^{*} \otimes \mathbb{1}\right) \operatorname{vec}(C)=\operatorname{vec}(F)
$$

which has the familiar form $A \boldsymbol{x}=\boldsymbol{b}$, where $\boldsymbol{x}=\operatorname{vec}(C)$. Now consider the case where $\Gamma>0$. The operation $\Delta(\cdot)$ is linear, thus the map $\operatorname{vec}(C) \mapsto \operatorname{vec}(\Delta(C))$ is a linear transformation. Therefore, there must be an $L^{2} \times L^{2}$ matrix $M$ corresponding to this transformation, defined trough

$$
M \operatorname{vec}(C)=\operatorname{vec}(\Delta(C))
$$

The transformation corresponding to $M$ deletes all elements in $\operatorname{vec}(C)$ corresponding to non-diagonal elements in $C$. In the vector $\operatorname{vec}(C)$, each diagonal element $C_{i i}$ is followed by $L$ non-diagonal elements, thus the matrix $M$ is a diagonal matrix of the form

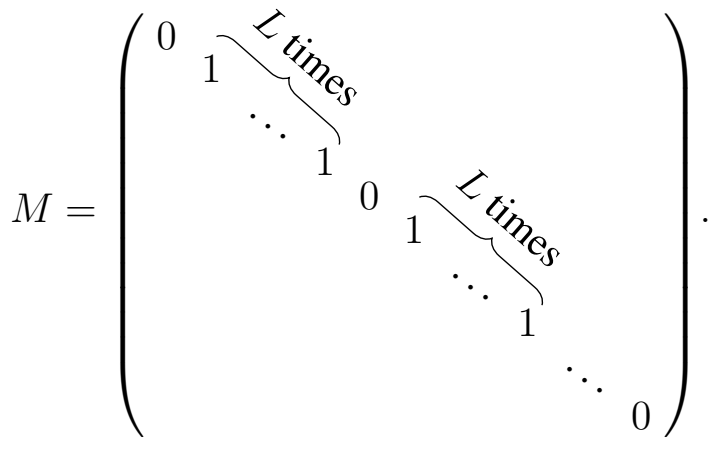

The diagonals of $M$ can also be conveniently written using modulo operation as

$$
M_{i i}= \begin{cases}0 & \text { if } i \bmod L+1=1 \\ 1 & \text { if } i \bmod L+1 \neq 1\end{cases}
$$


where $i \bmod L+1$ is the remainder of the integer division of $i$ by $L+1$. With this definition of $M$ in hand, the vectorized version of Eq. (8.11) is

$$
\left(\mathbb{1} \otimes W+W^{*} \otimes \mathbb{1}+\Gamma M\right) \operatorname{vec}(C)=\operatorname{vec}(F)
$$

which again is in the form $A \boldsymbol{x}=\boldsymbol{b}$.

The following Mathematica function was used to compute the NESS in the presence of dephasing in our simulations:

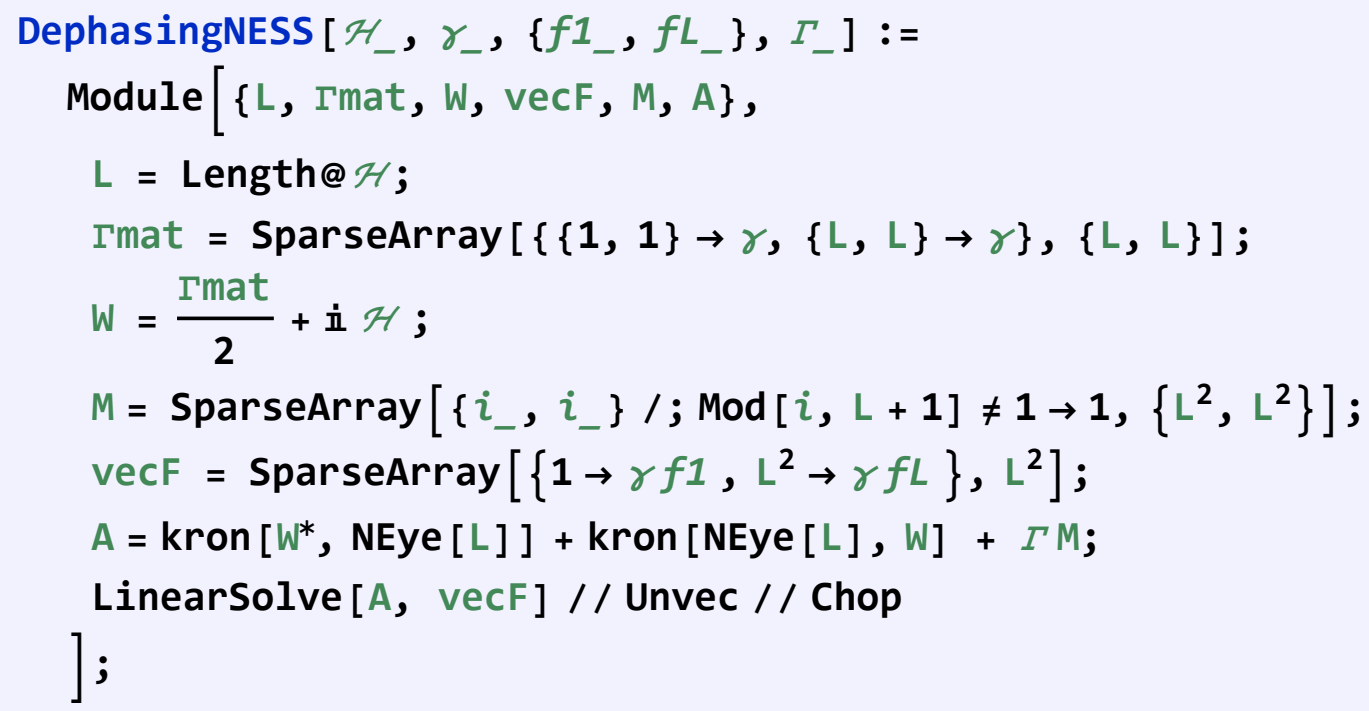

This code uses the functions kron, NEye and Unvec, which are part of the Qulib library [57], a Mathematica library for Quantum Information tasks developed by Prof. Landi. The core of the computation is done via the Mathematica bult-in method LinearSolve. Since the system does not exhibit any particular structure that can be exploited, besides being sparse, the computational cost is much higher in comparison with the solution of the Lyapunov equation. For this reason, the maximum system size we were able to simulate is smaller when dephasing is present.

\subsection{Transport properties with dephasing}

In this section, we will study the effect of dephasing in the transport properties of the system. When the on-site potential is constant, or, without loss of generality, identically zero, the covariance matrix in the NESS can be found analytically. Using the same ansatz 
(7.7), we obtain the following equation for the matrix $D$ in the NESS::

$$
i[H, D]+\frac{1}{2}\{D, \Gamma\}+\Gamma \Delta(D)=\tilde{\Gamma} .
$$

An identical system is solved in Ref. [8], where the authors studied the effect of dephasing on a boundary-driven bosonic chain. According to that solution, the analytical expression for the current in the NESS is

$$
J=\frac{2 \gamma\left(f_{1}-f_{L}\right)}{4+\gamma^{2}+\gamma \Gamma(L-1)}
$$

Notice that when $\Gamma$ is set to zero, the expression for the current without dephasing [(7.13)] is recovered.

When $L$ is large the constant terms in the denominator can be neglected, causing the current to scale as

$$
J \sim\left(\frac{2}{\Gamma}\right) \frac{\Delta f}{L}, \quad L \gg 1
$$

which is inversely proportional to $L$. Therefore, in the presence dephasing the transport is diffusive and the current obeys Fourier's law. Additionally, Eq. (8.21) also shows that this happens for any non-zero $\Gamma$, as long as $L$ is sufficiently large. This behavior is illustrated in Fig. 8.1. For all the values of $\Gamma$, the corresponding curve reaches the diffusive scaling [(8.22)], indicated by the dashed line. However, the smaller the value of $\Gamma$, the larger is the size range in which the dephasing-induced diffusion sets in. Notice, in particular, the curve for $\Gamma=10^{-3}$, represented in orange. For small values of $L$, the current is almost constant, similarly to the ballistic case, and the dephasing regime as achieved only for sizes of the order $\sim 10^{5}$.

Fig. 8.1 shows that that finite size effects play an important role in the transport regime of the system. It also indicates that there exists a crossover size $L_{\Gamma}$, which increases with $\Gamma$, above which the dephasing-induced diffusion dominates. Below this value, the transport regime is still influenced by the original Hamiltonian.

This characteristic length can be estimated using a simple scaling argument, similar to the one we used in section 7.2. Firstly, we notice that the coupling $\Gamma$ determines a 


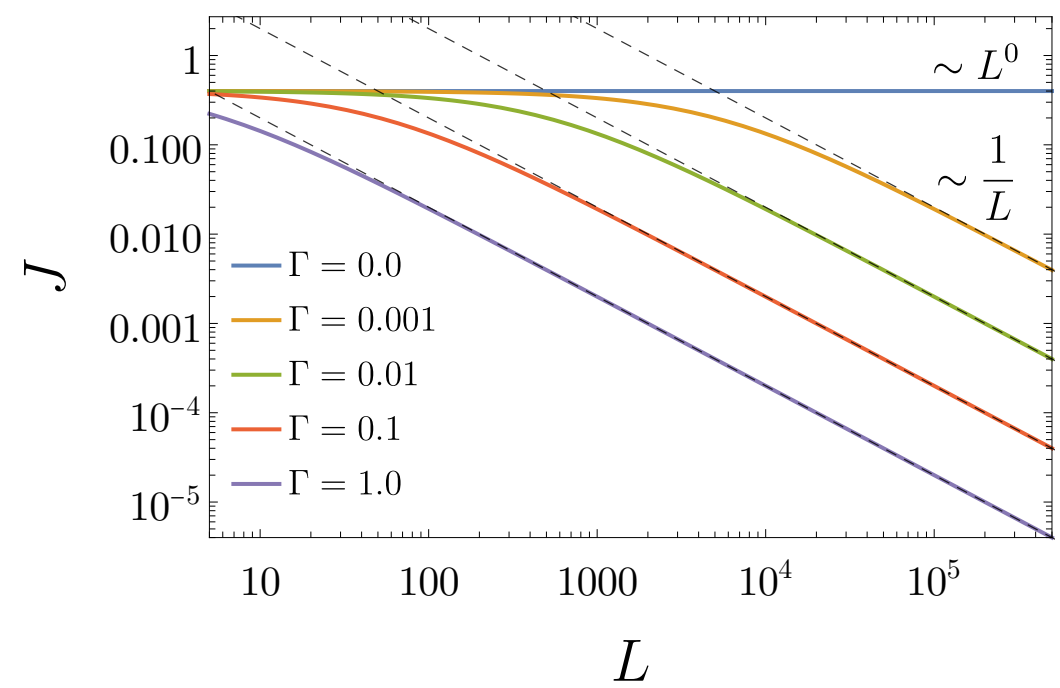

Figure 8.1: Scaling of the particle current with $L$ with zero on-site potential, for increasing values of $\Gamma$. All the curves eventually reach the diffusive scaling $\sim L^{-1}$, indicated by the dashed line.

characteristic time for the dephasing effect, given by $\tau_{\Gamma} \sim 1 / \Gamma$. This can be seen by inspecting the time evolution of the coherences in the single spin case with dephasing [(5.21)]. In this equation, the constant $\Gamma$ is the rate of relaxation. Then, this characteristic time may be compared with the time it takes for an excitation to traverse the whole chain, $\tau \sim 1 / L^{\alpha}$. If $\tau_{\Gamma} \ll \tau$, then the dephasing effect completely dominates, but if $\tau_{\Gamma} \gg \tau$ the transport properties are still affected by the Hamiltonian. The crossover length is thereby obtained by equating these two characteristic times, which results in

$$
L_{\Gamma} \sim \Gamma^{-1 /(\nu+1)}
$$

where we used the relation $\alpha=1 /(\nu+1)$. Notice that this derivation relies on the assumption that there is a single scaling coefficient.

To conclude this section, we will make a few remarks about Eq. (8.22), and precisely what it means to say that it follows Fourier's law. The original law states that the heat current in a piece of material is proportional to the temperature gradient [(1.1)]. In our ongoing example of the metal bar, the temperature profile in the NESS is a simple linear interpolation between $T_{1}$ and $T_{2}$ :

$$
T(x)=T_{1}+\left(\frac{T_{2}-T_{1}}{L}\right) x .
$$


Thus, by Eq. (1.1), the heat current in the NESS is given by

$$
J=\kappa \frac{\Delta T}{L}
$$

where $\Delta T_{1}-T_{2}$. Therefore, for a fixed value of $L$, the current is proportional to the temperature difference. In Eq. (8.22), however, when $L$ is fixed the current is instead proportional to the difference $f_{1}-f_{L}$. This is in agreement with the discussion we made in section 6.1, where we introduced our choice of local master equations. As we mentioned, in this dissertation we avoid providing a definition of the temperature inside the chain, and refer to $\Delta f$ simply as a "bias".

Therefore, we say that Eq. (8.22) satisfies Fourier's law by a simple analogy, with $\Delta f$ playing the role of $\Delta T$. It is worth mentioning that, in the particular case of a free chain with dephasing, the authors of Ref. [8] have indeed tried to define an internal temperature, but we will not dive into the subtleties of this discussion.

The above statements can me made more precise in the spin chain framework. In this case, the analogous of Fourier's law is Fick's law of diffusion, which states that the spin current is proportional to the magnetization gradient:

$$
J=-D \nabla\left\langle\sigma_{i}^{z}\right\rangle
$$

Here, $D$ is the diffusion constant, which is the analogous of the conductivity in Fourier's law. Furthermore, the two laws are, in fact, completely equivalent. This can be seen as follows. For large $L$, the gradient of the magnetization is given by

$$
\nabla\left\langle\sigma_{i}^{z}\right\rangle \approx \frac{\left\langle\sigma_{L}^{z}\right\rangle-\left\langle\sigma_{1}^{z}\right\rangle}{L}=\frac{\left\langle n_{1}\right\rangle-\left\langle n_{L}\right\rangle}{L}
$$

Imposing the continuity equation on the boundaries [Eqs. (6.29) and (6.30)], we obtain

$$
\gamma\left(f_{1}-\left\langle n_{1}\right\rangle\right)=\gamma\left(f_{L}-\left\langle n_{L}\right\rangle\right)
$$

whence $\left\langle n_{1}\right\rangle-\left\langle n_{L}\right\rangle=f_{1}-f_{L}$. Therefore,

$$
\nabla\left\langle\sigma_{i}^{z}\right\rangle \approx \frac{\Delta f}{L}
$$


which shows that the two laws are equivalent.

\subsection{Quasiperiodic chains with dephasing}

In this section, we will discuss the effect of dephasing when the quasiperiodic potentials are included. In this case, an analytical expression for the current can no longer be found, thus we applied the numerical method described in Sec. 8.2.

The transport properties of the AAH model with dephasing are summarized in Fig. 8.2. In this figure, each panel shows the scaling of the current for a fixed value of $\lambda$. As can be seen, the presence of dephasing always lead to diffusion if $L$ is sufficiently large. Quite remarkably, for $\lambda=1.1$, which corresponds to the localized phase, the dephasing noise restores the transport. A similar result has been reported in Ref. [56]. There, it was shown that a disordered $X X$ chain, which is localized, also becomes diffusive in the presence of dephasing.

Notice also that for $\lambda=0.1$, which is shown in panel (a), the length scale necessary for the diffusive behavior to be achieved is much higher than the other cases. This fact is explained by Eq. (8.23), as follows. When the system is ballistic, which corresponds to $\alpha=1$, the crossover length scales as $L_{\Gamma} \sim 1 / \Gamma$, and thus can be considerably large when $\Gamma$ is small. However, in the localized phase of the model, $\alpha=0$, and hence $L_{\Gamma} \approx 1$. This means that the dephasing effect dominates even in the smallest length scales.

In the case where $\lambda=0.9$, shown in panel (b), the eigenstates are also in the extended phase. However, the value of the current decreases slightly in the small length scale, before reaching a constant plateau. This is a consequence of the fact that the localization transition is not sharp for finite $L$, but becomes sharper when $L$ is increased.

The results for the Fibonacci model are shown in Fig. 8.3. Similarly to the AAH model, the addition of dephasing always leads to diffusive transport, but for smaller values of $\lambda$, the crossover length is higher. For $\lambda=4.0$, the Fibonacci model without dephasing exhibits subdiffusive transport, but with the addition of dephasing it becomes diffusive. This fact is already an indicative that dephasing can lead to an enhanced transport in the subdiffusive phase. This will be discussed in details in the next section. 

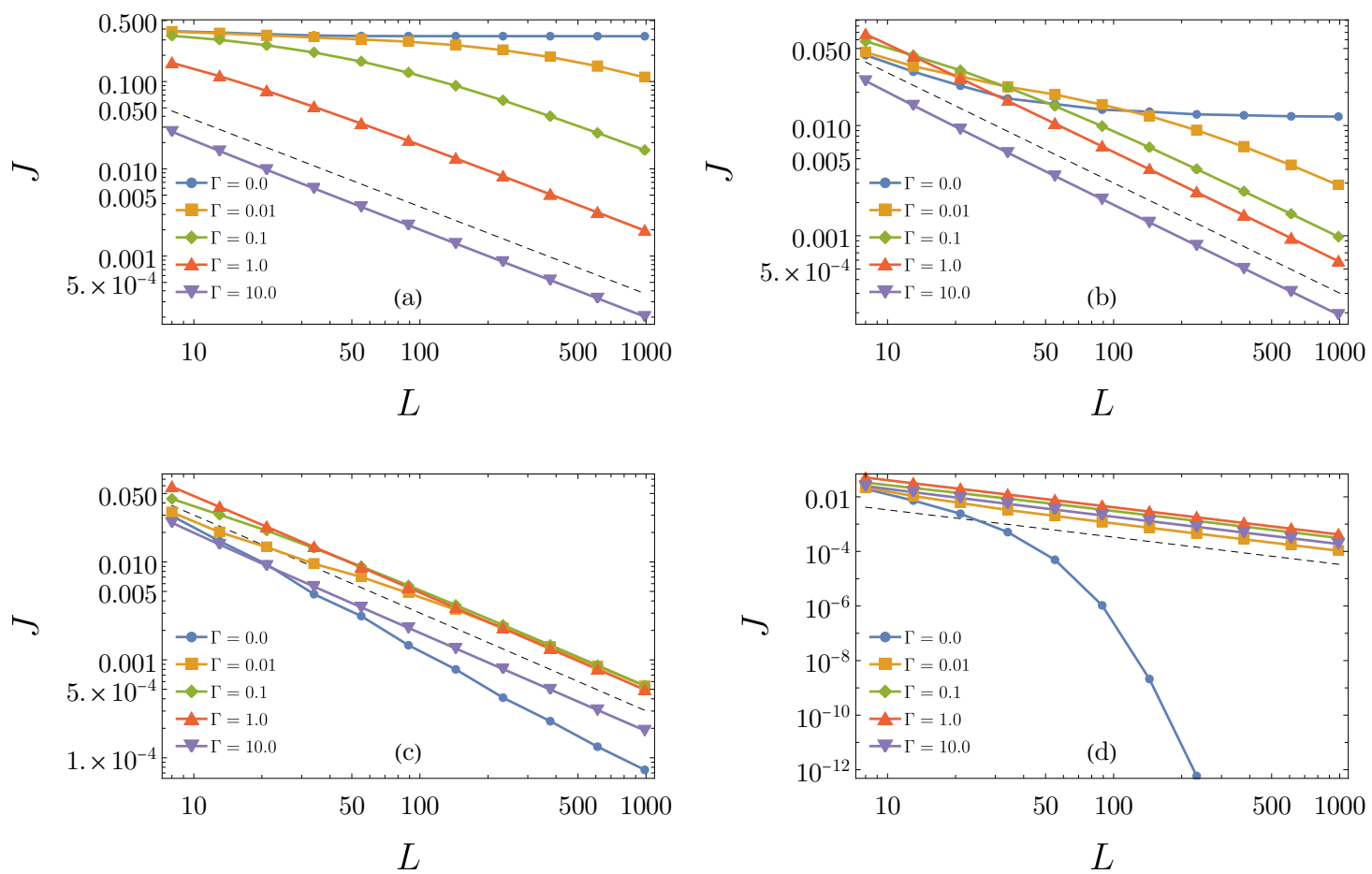

Figure 8.2: Scaling of the currents with system size for the AAH model with different dephasing strengths $\Gamma$. (a) $\lambda=0.1$. (b) $\lambda=0.9$. (c) $\lambda=1.0$. (d) $\lambda=1$.1. The dashed line is a visual reference of diffusive behavior, showing the curve $J=L^{-1}$. The current is averaged for 100 values of $\theta$ evenly spaced in between 0 and $\pi$. The other parameters are $\gamma=1, f_{1}=1$ and $f_{L}=0$. The system sizes are Fibonacci numbers.

\subsection{Scaling of the conductivity}

In this section, we will discuss the main original contribution of this dissertation, which is the study of the interplay between the strength of the quasiperiodic potential, $\lambda$, and the coupling to the dephasing baths, $\Gamma$. To do so, we will apply the same approach of Ref. [25].

As we discussed in section 8.3, the presence of dephasing always leads to diffusive transport. That is, for asymptotically large $L$, the current is inversely proportional to $L$, thus obeying Fourier's law,

$$
J=\frac{\kappa \Delta f}{L}, \quad L \gg 1 .
$$

where $\kappa$ is the conductivity. This constant can be explicitly defined by inverting the equation above, which results in 

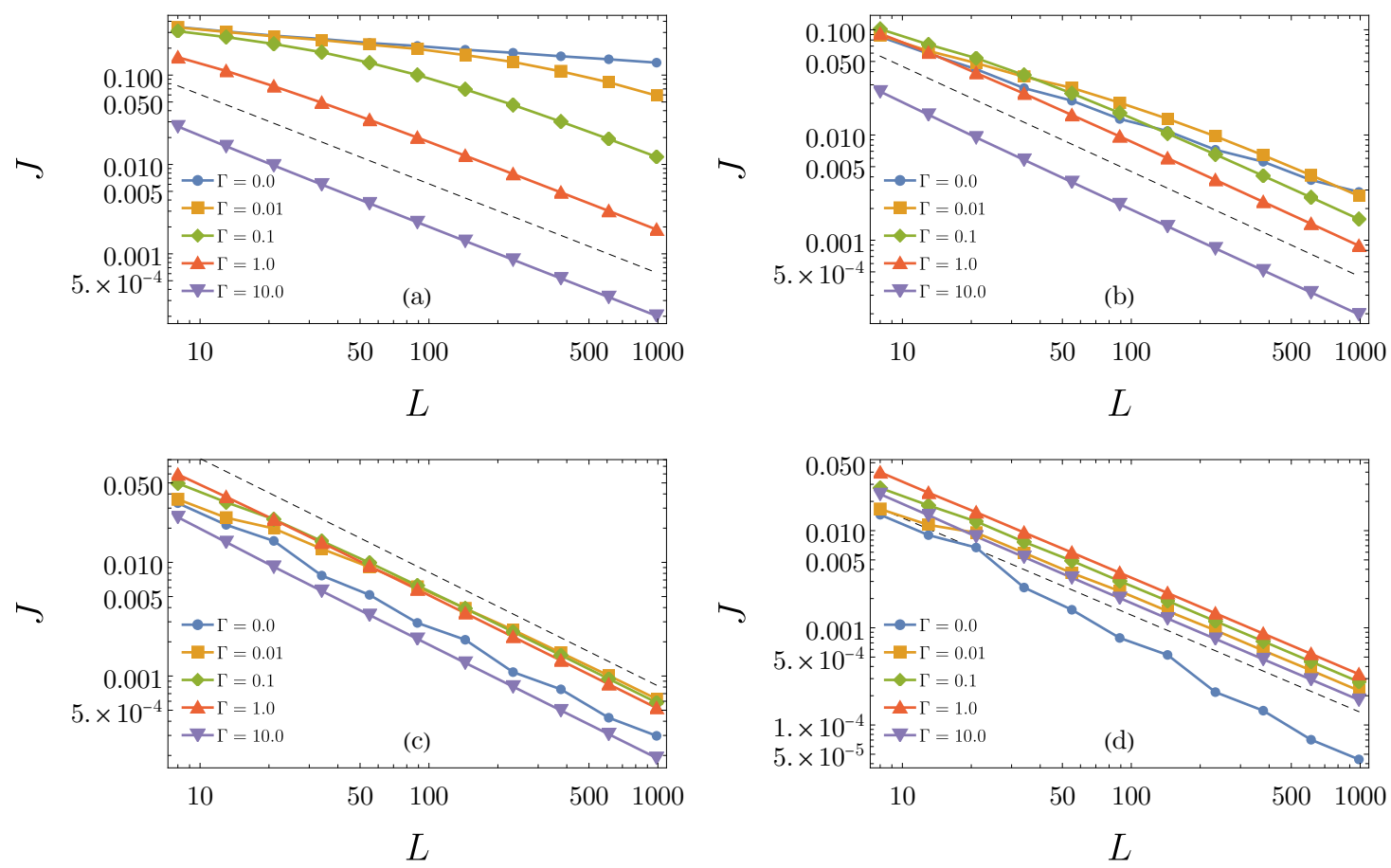

Figure 8.3: Scaling of the currents with system size for Fibonacci model with different dephasing strengths $\Gamma$. (a) $\lambda=0.5$. (b) $\lambda=1.0$. (c) $\lambda=2.0$. (d) $\lambda=4.0$. The dashed line is a visual reference of diffusive behavior, showing the curve $J=L^{-1}$. The other parameters are $\gamma=1, f_{1}=1$ and $f_{L}=0$. The system sizes are Fibonacci numbers.

$$
\kappa=\lim _{L \rightarrow \infty} \frac{L J(L)}{\Delta f}
$$

where $L \rightarrow \infty$ is to be understood as the thermodynamic limit. However, this definition only works in this case, in which the transport is diffusive. Imposing it to a ballistic regime, for example, would yield $\kappa \rightarrow \infty$.

Nonetheless, a generalized version of Fourier's law can still be imposed to nondiffusive systems, as long as $\kappa$ is allowed to have a dependence with $L$ :

$$
J \sim \frac{\kappa(L) \Delta f}{L}
$$

which is defined for $L$ large, but not infinite. For the general scaling $J \sim L^{\nu}$, we then have

$$
\kappa(L) \sim L^{1-\nu} .
$$


The "non-diffusive" factor of the scaling is simply hidden inside $\kappa(L)$. With this generalized definition, a diverging $\kappa$ can be understood as the defining feature of ballistic transport.

When dephasing is included, the conductivity is expected to have an additional dependence with $\Gamma$, i.e, $\kappa=\kappa(\Gamma, L)$. As we discussed in section 8.3, the dephasing effect only becomes important above a crossover length $L_{\Gamma}$. When $\Gamma$ is small, by Eq. (8.23), $L_{\Gamma} \gg 1$. Therefore, it is expected that the conductivity also exhibits two different scalings:

$$
\kappa(\Gamma, L)=\left\{\begin{array}{ll}
c L^{1-\nu} & L \leq L_{\Gamma} \\
\kappa_{\mathrm{deph}}(\Gamma) & L>L_{\Gamma}
\end{array},\right.
$$

where $c$ is some irrelevant proportionality factor. When the system size is bellow $L_{\Gamma}$, the transport follows the scaling dictated by the Hamiltonian without dephasing, hence $\kappa(\Gamma, L) \sim L^{1-\nu}$. When $L>L_{\Gamma}$, the dephasing effect dominates, thus $\kappa$ converges to some value $\kappa_{\text {deph }}(\Gamma)$, which is independent of $L$. By imposing continuity at $L_{\Gamma}$, one obtains

$$
\kappa_{\mathrm{deph}}(\Gamma) \sim L_{\Gamma}^{1-\nu} \sim \Gamma^{(\nu-1) /(\nu+1)}, \quad \Gamma \ll 1
$$

where we used the expression for $L_{\Gamma}$ [Eq. (8.23)]. When $\Gamma$ is large, Eq. (8.23) predicts that $L_{\Gamma} \approx 1$. Therefore, the diffusive transport induced by dephasing sets in even for small $L$, except in the shortest length scales.

Eq. (8.35) holds for $\Gamma \ll 1$. Conversely, the behavior for large $\Gamma$ can be obtained by expanding Eq. 8.35 in the limit of large $\Gamma$, which results in

$$
\kappa_{\mathrm{deph}}(\Gamma) \sim \frac{1}{\Gamma}, \quad \Gamma \gg 1
$$

This is, for example, the proportionality constant found in Eq. (8.21) (with an extra, irrelevant factor of 2).

For the remainder the text, we wil consider only the case where $L$ is large, well above the crossover length $L_{\Gamma}$. For this reason, we will drop the subscript "deph" and refer to the conductivity simply as $\kappa$.

When $\Gamma \rightarrow \infty$, it is expected that $\kappa \sim 1 / \Gamma$, as in Eq. (8.36). In contrast, when $\Gamma$ is 
sent to zero, the transport properties of the system without dephasing should be recovered, and $\kappa$ should scale according to Eq. (8.35). Therefore, the scaling of $\kappa$ with $\Gamma$ be used to detect the influence of the original Hamiltonian in the transport properties, even in the presence of dephasing. For example, if the original system without dephasing is ballistic, then we expect $\kappa$ to scale as $\sim \Gamma$, diverging in the limit $\Gamma \rightarrow 0$. In this manner, the diverging conductivity of ballistic transport is recovered. On the other hand, if the original system is subdiffusive, with some coefficient $\nu>1$, then $\kappa$ should scale as $\sim \Gamma^{\beta}$, where $\beta=(\nu-1) /(\nu+1)$, which vanishes in the limit $\Gamma \rightarrow 0$, as expected for subdiffusive transport.

We applied this framework to both the AAH and the Fibonacci models, with special interest in the behavior of the conductivity in their subdiffusive phase. In order to compute the conductivity as close as possible to the thermodynamic limit, we numerically found the current in the NESS for the largest system we are able to simulate with dephasing. Then, using Eq. (8.31) and the parameters $f_{1}=0$ and $f_{L}=0$, the conductivity is computed simply as $\kappa=J L$.

In Fig. 8.4 (a), we show the scaling of the conductivity in the Fibonacci model for a series of values of $\lambda$. As can be seen, when $\Gamma$ is large all curves reach the scaling $\sim 1 / \Gamma$, regardless of the value of $\lambda$. In contrast, when $\Gamma$ is sent to zero, the conductivity scales very differently depending on the coefficient $\nu$ of the original model without dephasing.

When $\lambda=0$, the original model without dephasing is ballistic, with $\nu=0$. As can be seen, the conductivity scales as $\sim \Gamma$ when $\Gamma$ is decreased, as predicted by Eq. 8.35. In the thermodynamic limit, the conductivity would diverge for $\Gamma \rightarrow 0$. However, since this curve was generated for a finite system with $L=987$, it saturates at a finite value.

For the values of $\lambda$ in the range $0<\lambda<3$, which corresponds to the superdiffusive phase of the model, the same observations hold, except that now, when $\Gamma$ is sent to zero, $\kappa$ grows with a smaller slope, determined by Eq. 8.35.

When $\lambda \approx 3$, the model without dephasing is very close to diffusive (as shown in Fig. 7.4). As one can see in Fig. 8.4 (a), $\kappa$ remains virtually constant when $\Gamma \rightarrow 0$, thus recovering the original conductivity of the model without dephasing. To be precise, for this value of $\lambda$ the Fibonacci model is not exactly diffusive, but slightly subdiffusive with $\nu=0.99$, hence the curve for $\kappa$ actually has a small, but non-zero inclination. The exact 
value of $\lambda$ that leads to diffusion is slightly above 3 .

The most interesting results correspond to the effect of dephasing on the subdiffusive phase of the model. In Fig. 8.4 (a), this corresponds to curves for $\lambda=4.0$ and $\lambda=5.0$, which are also highlighted separately in Fig. 8.4 (b). As one can see, the two curves also collapse in limit of large $\Gamma$, and start to follow the scaling $\sim 1 / \Gamma$. On the other hand, then $\Gamma$ is sent to zero, the conductivity decreases, following the scaling $\kappa \sim \Gamma^{\beta}$, and thus vanishes when $\Gamma \rightarrow 0$ (in the thermodynamic limit).

Interestingly, the coefficient $\beta$ closely matches the one predicted by Eq. 8.35, despite the fact that the Fibonacci model breaks the single coefficient hypothesis. This is illustrated in Fig. 7.1, which compares the coefficient $\beta$ computed in this manner with $(\nu-1) /(\nu+1)$, where $\nu$ is the corresponding transport coefficient without dephasing, computed numerically in section 7.4. At the time this dissertation was finished, we still have not found a reasonable explanation to this fact, but hope to do so before this result is published.

Notice that, in the diffusive phase of the model, the conductivity vanishes in both limits $\Gamma \rightarrow 0$ and $\Gamma \rightarrow \infty$, but for different physical reasons. For $\Gamma \rightarrow 0$, the original model without dephasing is recovered, and the conductivity is zero by the generalized definition of Fourier's law [Eq. (8.32)]. Conversely, when $\Gamma \rightarrow \infty$, the conductivity becomes very small due to the extreme effect of the dephasing noise, and the effect of the original Hamiltonian in the transport is essentially washed out. However, for intermediate values of $\Gamma$, there occurs a competition between the two effects.

As can be seen in Fig. 8.4 (b), the conductivity reaches a local maximum for a certain value of $\Gamma$, before the diffusive regime dominates. In this region, the effects of dephasing and of the Hamiltonian are balanced. As a consequence, for finite values of $L$, the absolute value of the current increases when $\Gamma$ is close to this value, with respect to the original current without dephasing. We refer to this phenomenon as dephasing-enhanced transport.

Fig. 8.6 shows the scaling of $\kappa$ with $\Gamma$ for the AAH model. The results are analogous to the Fibonacci model. For large $\Gamma$, the conductivity always reaches the diffusive scaling $\kappa \sim 1 / \Gamma$, independently of the value of $\lambda$. For small $\Gamma$, however, the behavior of $\kappa$ depends on $\lambda$. When $\lambda<1$, the AAH model is ballistic, so $\kappa$ diverges when $\Gamma \rightarrow 0$. 

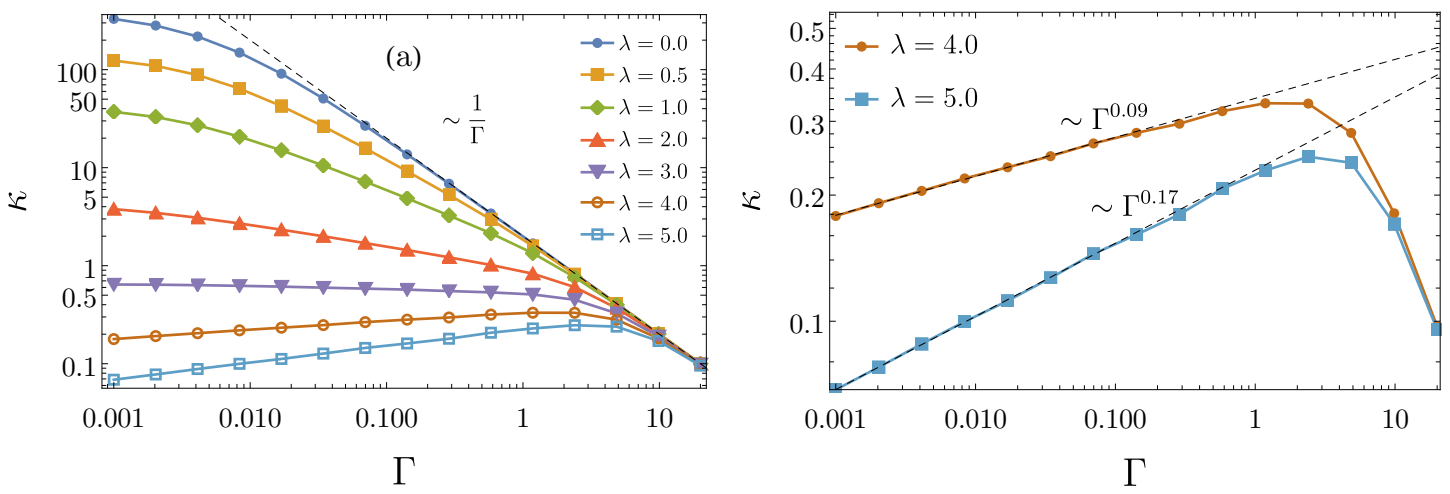

Figure 8.4: (a) Scaling of the conductivity with $\Gamma$ in the Fibonacci model for increasing values of $\lambda$. (b) Scaling of $\kappa$ for $\lambda=4.0$ and $\lambda=5.0$, which correspond to subdiffusive transport without dephasing.

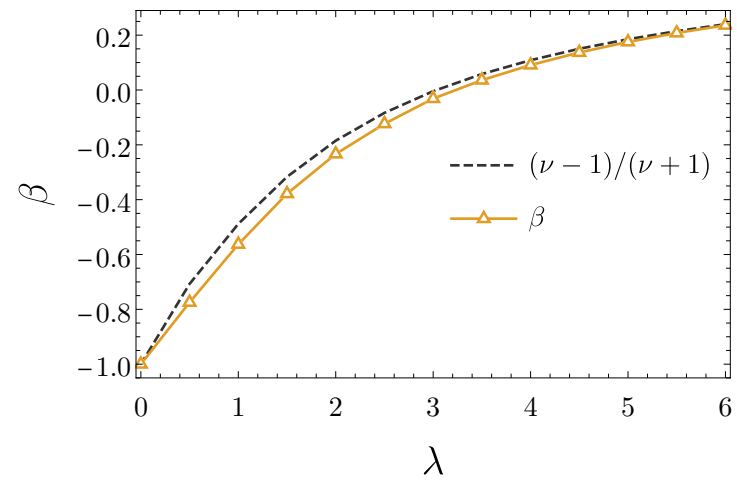

Figure 8.5: Coefficient $\beta$ fitted from the relation $\kappa \sim \Gamma^{\beta}$ in small $\Gamma$ region. The dashed line shows the value predicted by Eq. (8.35).

For $\lambda>1$, the system is localized, which corresponds to an extreme case of subdiffusive transport, where $\nu=\infty$; hence $\kappa$ vanishes when $\Gamma \rightarrow 0$. For intermediate values of $\Gamma$, before the transition to the diffusive regime, a maximum of $\kappa$ is reached, similarly to what occurs in the subdiffusive phase of the Fibonacci model.

At the critical point, the model is subdiffusive without dephasing. When the current is averaged over $\theta$, the transport coefficient is $\nu=1.26$, while for the symmetrical version of the potential it is $\nu=1.1$. In both cases, as shown in Fig. 8.6 (b), the conductivity decreases when $\Gamma$ is decreased. In the thermodynamic limit, $\kappa$ should vanish, but since $L$ is finite, it converges to the non-zero value of the current.

However, in neither case the coefficient $\beta$ matches the value predicted by Eq. (8.35), even for the symmetrical potential, for which the connection between the coefficients [(7.5)] holds. For the symmetrical case, the coefficient is $\nu=1.1$, which by Eq. (8.35) 
should result in $\beta \approx 0.09$. However, the coefficient we found is approximately 0.14 . At time this dissertation was finished, we still have not found an explanation to this discrepancy.
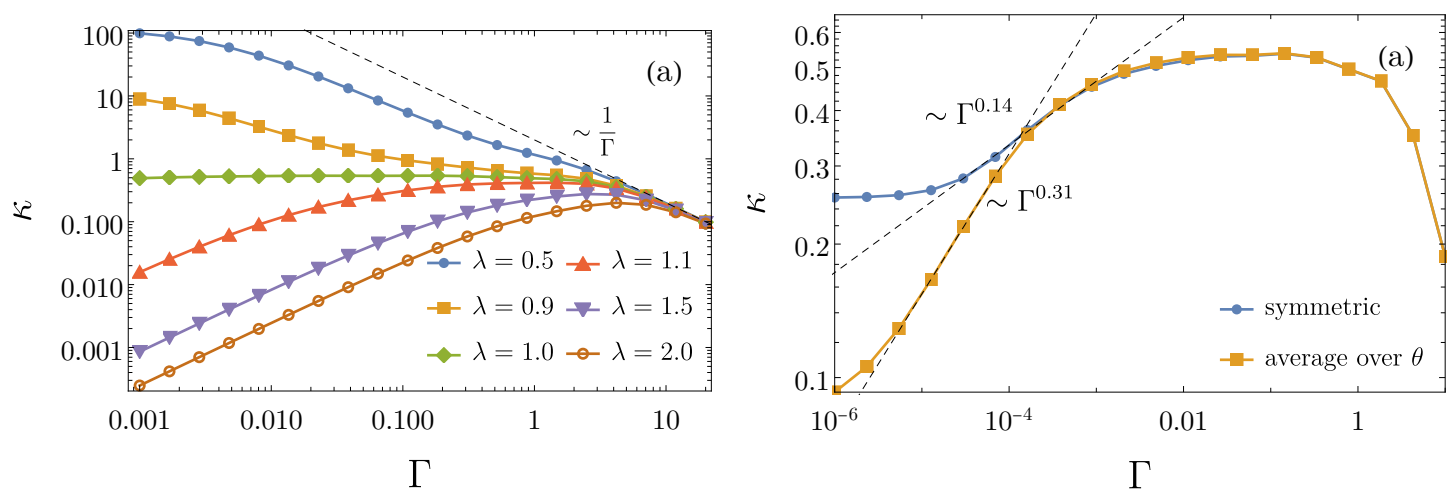

Figure 8.6: (a) Scaling of the conductivity with $\Gamma$ in the AAH model for increasing values of $\lambda$. (b) Comparison of the scaling of $\kappa$ with $L$ in the critical point between between the symmetrical version of the potential and the average over $\theta$. This particular curve was generate for a smaller system size, $L=610$. 


\section{Chapter 9}

\section{Conclusion}

In this dissertation we have studied the transport properties of non-interacting fermionic chains subject to two choices of quasiperiodic potentials: the Aubry-André-Harper and the Fibonacci model. We considered both isolated and boundary-driven chains, with a particular focus on the latter.

In the closed system scenario, we classified the transport regime of the system via the scaling of the root mean square deviation of the wave function with time during a unitary time evolution, starting from a localized wave-packet. To do so, the wave function at each time step was computed numerically, using an efficient code written in Mathematica, which is provided in section 4.1. The transport coefficients we obtained are consistent with those found in the literature.

Then, we discussed our main class of models, the boundary-driven quantum chains, obtained by coupling local Linbdlad dissipators at each end. In the NESS, the covariance matrix obey a Lyapunov equation [(6.17)]. We have written efficient functions to solve this equation, provided in section 6.3. We have found that, for our particular system, the algorithm we used outperforms the usual procedure to solve the Lyapunov equation, the Bartels-Stewart algorithm, at least in the parameter region we worked. The full code is still being adjusted, but will be freely available upon completion.

Then, using this numerical method, we studied the non-equilibrium transport properties of the boundary-driven chains with quasiperiodic potentials. In this scenario, we classified the transport regime via the scaling of the particle current with the system size. As in the coherent transport case, we have found transport coefficients consistent with the 
values reported in the literature.

Finally, in chapter 8, we considered the effect of dephasing noise on the boundarydriven chains. We verified that the presence of dephasing always leads to diffusive transport in the thermodynamic limit, regardless of the original transport properties of the system. Nevertheless, we have verified that by analyzing the scaling of the conductivity with the coupling to the dephasing, it is possible to probe the original transport coefficient of the system, even when dephasing is present.

Furthermore, we have shown that the presence of dephasing may lead to an increased current in the subdiffusive phases of the AAH and Fibonacci models, when compared to the zero dephasing case. This is the main original contribution of this dissertation, as it corresponds to a realization of dephasing-assisted transport in quasi-periodic chains. These results are currently being prepared for publication. 


\section{Appendix A}

\section{The Jordan-Wigner Transformation}

The Jordan-Wigner transformation maps spin operators into fermionic operators. Consider the Hamiltonian for a $X X$ spin chain with open boundary conditions:

$$
\begin{aligned}
\mathcal{H} & =2 J \sum_{i=1}^{L-1}\left(\sigma_{i}^{x} \sigma_{i+1}^{x}+\sigma_{i}^{y} \sigma_{i+1}^{y}\right)+\frac{1}{2} \sum_{i}^{L} V_{i} \sigma_{i}^{z} \\
& =J \sum_{i=1}^{L-1}\left(\sigma_{i}^{+} \sigma_{i+1}^{-}+\sigma_{i}^{-} \sigma_{i+1}^{+}\right)+\frac{1}{2} \sum_{i}^{L} V_{i} \sigma_{i}^{z} .
\end{aligned}
$$

The Jordan-Wigner transformation is defined as

$$
c_{i}=\left[\bigotimes_{k=1}^{i-1}\left(-\sigma_{k}^{z}\right)\right] \sigma_{i}^{-}
$$

The term $\left(-\sigma_{1}^{z}\right) \otimes \cdots \otimes\left(-\sigma_{i-1}^{z}\right)$ is known as a Jordan-Wigner string. Notice that all the operators in the string act on different sites and thus commute with each other. One may show that the operators defined in Eq. A. 2 satisfy the usual fermionic algebra, i.e,

$$
\left\{c_{i}, c_{j}^{\dagger}\right\}=\delta_{i j} \quad \text { and } \quad\left\{c_{i}, c_{j}\right\}=\left\{c_{i}^{\dagger}, c_{j}^{\dagger}\right\}=0
$$


Using this transformation, the operator $c_{i}^{\dagger} c_{i}$ can then be written as follows:

$$
\begin{aligned}
c_{i}^{\dagger} c_{i} & =\left[\bigotimes_{k=1}^{i-1}\left(-\sigma_{k}^{z}\right)\right] \sigma_{i}^{+}\left[\bigotimes_{k=1}^{i-1}\left(-\sigma_{k}^{z}\right)\right] \sigma_{i}^{-} \\
& =\left[\bigotimes_{k=1}^{i-1}\left(\sigma_{k}^{z}\right)^{2}\right] \sigma_{i}^{+} \sigma_{i}^{-} \\
& =\sigma_{i}^{+} \sigma_{i}^{-},
\end{aligned}
$$

where we used the facts that spin operators acting on different sites commute and $\left(\sigma_{i}^{z}\right)^{2}=$ 1. Since $\sigma_{i}^{z}=2 \sigma_{i}^{+} \sigma_{i}^{-}-1$, we thus have that

$$
\sigma_{i}^{z}=2 c_{i}^{\dagger} c_{i}+1
$$

Similarly, the operator $c_{i}^{\dagger} c_{i+1}$ can be written in terms of spin operators as follows:

$$
\begin{aligned}
c_{i}^{\dagger} c_{i+1} & =\left[\bigotimes_{k=1}^{i-1}\left(-\sigma_{k}^{z}\right)\right] \sigma_{i}^{+}\left[\bigotimes_{k=1}^{i}\left(-\sigma_{k}^{z}\right)\right] \sigma_{i+1}^{-} \\
& =\left[\bigotimes_{k=1}^{i-1}\left(\sigma_{k}^{z}\right)^{2}\right] \sigma_{i}^{+}\left(-\sigma_{i}^{z}\right) \sigma_{i+1}^{-} \\
& =\sigma_{i}^{+} \sigma_{i+1}^{-}
\end{aligned}
$$

where we used the fact that $\sigma_{i}^{-} \sigma_{i}^{z}=-\sigma_{i}^{-}$. The adjoint is given by $c_{i}^{\dagger} c_{i+1}=\sigma_{i}^{-} \sigma_{i+1}^{+}$. Therefore, the $X X$ chain Hamiltonian can then be written as

$$
\mathcal{H}=-J \sum_{i=1}^{L-1}\left(c_{i}^{\dagger} c_{i+1}+c_{i+1}^{\dagger} c_{i}\right)+\frac{1}{2} \sum_{i=1}^{L} V_{i}\left(2 c_{i}^{\dagger} c_{i}+1\right)
$$

which, up to an irrelevant constant factor, is equal to the tight-binding Hamiltonian [Eq. 2.1]. 


\section{Bibliography}

[1] F. Bonetto, J. L. Lebowitz, and L. Rey-Bellet, "Fourier's Law: a Challenge for Theorists," vol. 0, no. 1, pp. 1-23, 2000. arXiv: 0002052 [math-ph]. [Online]. Available: http://arxiv.org/abs/math-ph/0002052.

[2] A. Dhar, "Heat transport in low-dimensional systems," Advances in Physics, vol. 57 , no. 5, pp. 457-537, 2008, ISSN: 00018732. DOI: 10 . $1080 / 00018730802538522$. arXiv: 0808.3256.

[3] Z. Rieder, J. L. Lebowitz, and E. Lieb, "Properties of a harmonic crystal in a stationary nonequilibrium state," Journal of Mathematical Physics, vol. 8, no. 5, pp. 1073-1078, 1967. DOI: 10.1063/1.1705319. eprint: https: / / doi . org/10.1063/1.1705319. [Online]. Available: https://doi.org/10. $1063 / 1.1705319$.

[4] E. Ilievski and J. De Nardis, "Microscopic origin of ideal conductivity in integrable quantum models," Phys. Rev. Lett., vol. 119, p. 020 602, 2 Jul. 2017. DOI: 10 . 1103 /PhysRevLett.119.020602. [Online]. Available: https: / / Iink . aps.org/doi/10.1103/PhysRevLett.119.020602.

[5] J. S. Ferreira and M. Filippone, "Ballistic-to-diffusive transition in spin chains with broken integrability," Jun. 2020. arXiv: 2006.13891 . [Online]. Available: http://arxiv.org/abs/2006.13891.

[6] M. Ljubotina, M. Žnidari, and T. Prosen, "Spin diffusion from an inhomogeneous quench in an integrable system," Nature Communications, vol. 8, no. 1, pp. 1-6, Jul. 2017, ISSN: 20411723. DOI: 10.1038 /ncomms 16117. arXiv: 1702.04210. [Online]. Available: www . nature.com/naturecommunications.

[7] M. Žnidarič, "Exact solution for a diffusive nonequilibrium steady state of an open quantum chain," Journal of Statistical Mechanics: Theory and Experiment, vol. 2010, no. 5, 2010, ISSN: 17425468. DOI: 10 . $1088 / 1742-5468 / 2010 /$ 05/L05002. arXiv: 1005.1271.

[8] A. Asadian, D. Manzano, M. Tiersch, and H. J. Briegel, "Heat transport through lattices of quantum harmonic oscillators in arbitrary dimensions," Physical Review E - Statistical, Nonlinear, and Soft Matter Physics, vol. 87, no. 1, pp. 1-8, 2013, ISSN: 15393755. DOI: 10.1103 / PhysRevE . 87 .012109. arXiv: 1204 . 0904.

[9] P. W. Anderson, “Absence of diffusion in certain random lattices," Physical Review, vol. 109, no. 5, pp. 1492-1505, Mar. 1958, ISSN: 0031899X. DOI: 10.1103 / PhysRev . 109 . 1492. [Online]. Available: https : / / journals.aps . org/pr/abstract/10.1103/PhysRev.109.1492. 
[10] S. Aubry and G. André, "Analyticity breaking and Anderson localization in incommensurate lattices," Proceedings, VIII International Colloquium on GroupTheoretical Methods in Physics, vol. 3, 1980.

[11] P. G. Harper, "Single band motion of conduction electrons in a uniform magnetic field," Proceedings of the Physical Society. Section A, vol. 68, no. 10, pp. 874-878, 1955, ISSN: 03701298. DOI: $10.1088 / 0370-1298 / 68 / 10 / 304$.

[12] S. Ostlund, R. Pandit, D. Rand, H. J. Schellnhuber, and E. D. Siggia, "One-dimensional schrödinger equation with an almost periodic potential," Physical Review Letters, vol. 50, no. 23, pp. 1873-1876, 1983, ISSN: 00319007. DOI: 10.1103 / PhysRevLett. 50.1873 .

[13] M. Kohmoto, B. Sutherland, and C. Tang, "Critical wave functions and a Cantorset spectrum of a one-dimensional quasicrystal model," Physical Review B, vol. 35, no. 3, pp. 1020-1033, 1987, ISSN: 01631829. DOI: 10.1103 / PhysRevB. 35. 1020.

[14] S. Abe and H. Hiramoto, Dynamics of an Electron in Qusiperiodic Systems. I. Fibonacci Model, 1988.

[15] H. HIRAMOTO and M. KOHMOTO, "Electronic Spectral and Wavefunction Properties of One-Dimensional Quasiperiodic Systems: a Scaling Approach," International Journal of Modern Physics B, vol. 06, no. 03n04, pp. 281-320, Feb. 1992, ISSN: 0217-9792. DOI: $10.1142 /$ s 0217979292000153.

[16] A. Purkayastha, S. Sanyal, A. Dhar, and M. Kulkarni, "Anomalous transport in the Aubry-André-Harper model in isolated and open systems," Physical Review B, vol. 97, no. 17, Feb. 2017. DOI: 10.1103/PhysRevB .97 . 174206. arXiv: 1702 . 05228. [Online]. Available: http : / / arxiv . org / abs / 1702 . $05228 \% 20$ http://dx.doi.org/10.1103/PhysRevB.97.174206.

[17] V. K. Varma, C. De Mulatier, and M. Žnidarič, "Fractality in nonequilibrium steady states of quasiperiodic systems," Physical Review E, vol. 96, no. 3, pp. 1-11, 2017, ISSN: 24700053. DOI: 10.1103 / PhysRevE . 96.032130 . arXiv: 1703 . 05844.

[18] J. E. Lye, L. Fallani, C. Fort, V. Guarrera, M. Modugno, D. S. Wiersma, and M. Inguscio, "Effect of interactions on the localization of a bose-einstein condensate in a quasiperiodic lattice," Phys. Rev. A, vol. 75, p. 061 603, 6 Jun. 2007. DOI: 10.1103/PhysRevA. 75.061603. [Online]. Available: https : / / ink . aps.org/doi/10.1103/PhysRevA.75.061603.

[19] R. Roth and K. Burnett, "Phase diagram of bosonic atoms in two-color superlattices," Phys. Rev. A, vol. 68, p. 023 604, 2 Aug. 2003. DOI: 10 . 1103 / Phy sRevA. 68.023604 . [Online]. Available: https : / / link.aps . org/doi/ 10 . 1103 /PhysRevA. 68.023604.

[20] B. Damski, J. Zakrzewski, L. Santos, P. Zoller, and M. Lewenstein, "Atomic bose and anderson glasses in optical lattices," Phys. Rev. Lett., vol. 91, p. 080403,8 Aug. 2003. DOI: 10.1103 / PhysRevLett. 91.080403 . [Online]. Available: https://1ink.aps.org/doi/10.1103/PhysRevLett.91.080403. 
[21] L. Tanzi, E. Lucioni, S. Chaudhuri, L. Gori, A. Kumar, C. D’Errico, M. Inguscio, and G. Modugno, "Transport of a bose gas in 1d disordered lattices at the fluidinsulator transition," Phys. Rev. Lett., vol. 111, p. 115 301, 11 Sep. 2013. DOI: 10 . 1103 /PhysRevLett.111.115301. [Online]. Available: https : / / Iink . aps.org/doi/10.1103/PhysRevLett.111.115301.

[22] J. Billy, V. Josse, Z. Zuo, A. Bernard, B. Hambrecht, P. Lugan, D. Clément, L. Sanchez-Palencia, P. Bouyer, and A. Aspect, "Direct observation of anderson localization of matter waves in a controlled disorder," Nature, vol. 453, pp. 891-4, Jul. 2008. DOI: $10.1038 /$ nature0 7000 .

[23] M. Bolsterli, M. Rich, and W. M. Visscher, "Simulation of nonharmonic interactions in a crystal by self-consistent reservoirs," Physical Review A, vol. 1, no. 4, pp. 1086-1088, Apr. 1970, ISSN: 10502947. DOI: 10.1103 / PhysRevA. 1 . 1086.

[24] M. Büttiker, "Small normal-metal loop coupled to an electron reservoir," Physical Review B, vol. 32, no. 3, pp. 1846-1849, Aug. 1985, ISSN: 01631829. DOI: 10 . 1103 /PhysRevB.32.1846.

[25] M. Žnidarič, J. J. Mendoza-Arenas, S. R. Clark, and J. Goold, "Dephasing enhanced spin transport in the ergodic phase of a many-body localizable system," Annalen der Physik, vol. 529, no. 7, Sep. 2016. DOI: 10.1002 / andp . 201600298. arXiv: 1609.09367 . [Online]. Available: http: / / arxiv.org/abs/1609. $09367 \% 20$ http://dx.doi.org/10.1002/andp.201600298.

[26] P. Jordan and E. Wigner, "Über das Paulische Äquivalenzverbot," Zeitschrift für Physik, vol. 47, no. 9-10, pp. 631-651, Sep. 1928, ISSN: 14346001. DOI: 10 . 1007 /BF 01331938.

[27] A. W, N. Ashcroft, N. Mermin, N. Mermin, and B. P. Company, Solid State Physics, ser. HRW international editions. Holt, Rinehart and Winston, 1976, ISBN: 9780030839931.

[Online]. Available: https : / / books . google . com . br/ books ? id= OXIfAQAAMAAJ.

[28] M. Segev, Y. Silberberg, and D. Christodoulides, "Anderson localization of light," Nature Photonics, vol. 7, pp. 197-204, Mar. 2013. DOI: 10.1038 / nphoton . 2013.30.

[29] M. Büttiker and M. Moskalets, "From anderson localization to mesoscopic physics," International Journal of Modern Physics B, vol. 24, Feb. 2010. DOI: 10.1142 / S0217979210064514.

[30] H. Hu, A. Strybulevych, J. Page, S. Skipetrov, and B. Tiggelen, "Localization of ultrasound in a three-dimensional elastic network," Nature Physics, vol. 4, May 2008. DOI: $10.1038 /$ nphys1101.

[31] H. Wang, R. Marsh, J. P. Lewis, and R. A. Römer, "CHAPTER 21 - Electronic transport and localization in short and long DNA," in Modern Methods for Theoretical Physical Chemistry of Biopolymers, E. B. Starikov, J. P. Lewis, and S. Tanaka, Eds., Amsterdam: Elsevier Science, 2006, pp. 407-427, ISBN: 978-0-444-522207. DOI: https://doi .org/10.1016/B978-044452220-7/50085- 
X. [Online]. Available: http: / / www. sciencedirect. com / science / article/pii/B978044452220750085X.

[32] E. Altman, "Many-body localization and quantum thermalization," Nature Physics, vol. 14, no. 10, pp. 979-983, Oct. 2018, ISSN: 1745-2481. DOI: 10 . 1038 / s41567-018-0305-7. [Online]. Available: https : / / doi . org / 10 . $1038 /$ s $41567-018-0305-7$.

[33] D. A. Abanin, E. Altman, I. Bloch, and M. Serbyn, "Colloquium: Many-body localization, thermalization, and entanglement," Rev. Mod. Phys., vol. 91, p. 021 001, 2 May 2019. DOI: 10.1103/RevModPhys.91.021001. [Online]. Available: https://link.aps.org/doi/10.1103/RevModPhys.91.021001.

[34] K. Ishii, "Localization of Eigenstates and Transport Phenomena in the One-Dimensional Disordered System*)," Progress of Theoretical Physics Supplement, vol. 53, pp. 77138, Jan. 1973, ISSN: 0375-9687. DOI: 10 .1143/PTPS.53 . 77. eprint: https: / / academic.oup.com/ptps/article-pdf/doi/10.1143/PTPS . 53.77/5426871/53-77.pdf. [Online]. Available: https:// doi.org/ $10.1143 / \mathrm{PTPS} .53 .77$.

[35] E. Abrahams, P. W. Anderson, D. C. Licciardello, and T. V. Ramakrishnan, "Scaling theory of localization: Absence of quantum diffusion in two dimensions," Physical Review Letters, vol. 42, no. 10, pp. 673-676, Mar. 1979, ISSN: 00319007. DoI: 10 . 1103 / PhysRevLett . 42 . 673. [Online]. Available: https : / / journals.aps.org/prl/abstract/10.1103/PhysRevLett. 42 . 673.

[36] H. Furstenberg and H. Kesten, "Products of random matrices," Ann. Math. Statist., vol. 31, no. 2, pp. 457-469, Jun. 1960. DOI: 10 . 1214 / aoms / 1177705909. [Online]. Available: https: / / doi .org/10.1214/aoms/1177705909.

[37] F. Evers and A. D. Mirlin, "Anderson transitions," Rev. Mod. Phys., vol. 80, pp. 13551417, 4 Oct. 2008. DOI: 10.1103 /RevModPhys . 80 . 1355. [Online]. Available: https: / / link . aps . org/doi / 10 . 1103/RevModPhys . 80 . 1355.

[38] N. F. MOTT, "Metal-insulator transition," Rev. Mod. Phys., vol. 40, pp. 677-683, 4 Oct. 1968. DOI: 10 . 1103 / RevModPhys . 40 . 677. [Online]. Available: https://link.aps.org/doi/10.1103/RevModPhys.40.677.

[39] M. Modugno, "Exponential localization in one-dimensional quasi-periodic optical lattices," New Journal of Physics, vol. 11, no. 3, p. 033 023, Mar. 2009. DOI: 10 . 1088/1367-2630/11/3/033023. [Online]. Available: https: / / doi . org/10.1088\%2F1367-2630\%2F112F32F033023.

[40] G. A. Domínguez-Castro and R. Paredes, "The Aubry-André model as the hobbyhorse for understanding localization phenomenon," Dec. 2018. DOI: $10.1088 /$ 1361-6404/ab1670. arXiv: 1812.06201. [Online]. Available: http: / / arxiv.org/abs/1812.06201\%20http://dx.doi.org/10.1088/ $1361-6404 / a b 1670$.

[41] S. Y. Jitomirskaya, "Metal-insulator transition for the almost Mathieu operator," Tech. Rep., 1999, pp. 1159-1175. 
[42] T. C. Halsey, M. H. Jensen, L. P. Kadanoff, I. Procaccia, and B. I. Shraiman, "Fractal measures and their singularities: The characterization of strange sets," Phys. Rev. A, vol. 33, pp. 1141-1151, 2 Feb. 1986. DOI: $10.1103 /$ PhysRevA. 33 . 1141. [Online]. Available: https : / / link .aps . org/doi/10.1103/ PhysRevA. 33.1141.

[43] F. Evers and A. D. Mirlin, "Fluctuations of the inverse participation ratio at the Anderson transition," Physical Review Letters, vol. 84, no. 16, pp. 3690-3693, Jan. 2000. DOI: 10.1103 /PhysRevLett.84.3690.arXiv: 0001086 [cond-mat] . [Online]. Available: http : / / arxiv . org / abs / cond-mat / $0001086 \%$ 20http://dx.doi.org/10.1103/PhysRevLett.84.3690.

[44] H. Yao, H. Khoudli, L. Bresque, and L. Sanchez-Palencia, "Critical Behavior and Fractality in Shallow One-Dimensional Quasiperiodic Potentials," Physical Review Letters, vol. 123, no. 7, Apr. 2019. DOI: 10 . 1103 / PhysRevLett . 123 . 070405. arXiv: 1904.01463 . [Online]. Available: http: / / arxiv.org / abs/1904.01463\%20http://dx.doi.org/10.1103/PhysRevLett. 123.070405 .

[45] G. Rubiano, "Properties and generalizations of fibonacci word fractal, exploring fractal curves.," The Mathematica Journal, vol. 16, pp. 1-25, Jan. 2014.

[46] D. Perrin and A. Restivo, "A note on sturmian words," Theoretical Computer Science, vol. 429, pp. 265-272, 2012, Magic in Science, ISSN: 0304-3975. DOI: https://doi.org/10.1016/j.tcs.2011.12.047. [Online]. Available: http: / / www . sciencedirect.com/science/article/pii/ s0304397511010139.

[47] P. Kalugin, A. Kitaev, and L. Levitov, "Electron spectrum of a one-dimensional quasicrystal," Sov. Phys. JETP, vol. 64, no. 2, p. 410, 1986.

[48] N. Macé, N. Laflorencie, and F. Alet, "Many-body localization in a quasiperiodic Fibonacci chain," SciPost Physics, vol. 6, no. 4, Nov. 2018. DOI: 10.21468 / ScipostPhys.6.4.050. arXiv: 1811.01912. [Online]. Available: http: / / arxiv.org/abs / 1811 .01912\%20http://dx . doi . org/ 10 . $21468 /$ scipostPhys.6.4.050.

[49] H. P. Breuer and F. Petruccione, The theory of open quantum systems. Great Clarendon Street: Oxford University Press, 2002.

[50] M. A. Nielsen and I. L. Chuang, Quantum Computation and Quantum Information: 10th Anniversary Edition, 10th. USA: Cambridge University Press, 2011, ISBN: 1107002176.

[51] G. Lindblad, "On the generators of quantum dynamical semigroups," Communications in Mathematical Physics, vol. 48, no. 2, pp. 119-130, Jun. 1976, ISSN: 00103616. DOI: 10.1007 /BF01608499.

[52] V. Gorini, "Completely positive dynamical semigroups of N-level systems," Journal of Mathematical Physics, vol. 17, no. 5, p. 821, Aug. 1976, ISSN: 00222488. DOI: $10.1063 / 1.522979$. [Online]. Available: http://scitation.aip. org/content/aip/journal/jmp/17/5/10.1063/1.522979. 
[53] J. P. Santos and G. T. Landi, "Microscopic theory of a nonequilibrium open bosonic chain," Phys. Rev. E, vol. 94, p. 062 143, 6 Dec. 2016. Dor: 10.1103 / Phy sRevE . 94.062143. [Online]. Available: https : / / link.aps . org / doi / 10 . 1103 /PhysRevE.94.062143.

[54] R. H. Bartels and G. W. Stewart, "Solution of the matrix equation $a x+x b=c$ [f4]," Commun. ACM, vol. 15, no. 9, pp. 820-826, Sep. 1972, ISSN: 0001-0782. DOI: $10.1145 / 361573.361582$. [Online]. Available: https: / / doi .org/10 . $1145 / 361573.361582$.

[55] B. Li and J. Wang, "Anomalous heat conduction and anomalous diffusion in one dimensional systems," Physical Review Letters, vol. 91, no. 4, Jun. 2003. DOI: 10.1103/PhysRevLett.91.044301. arXiv: 0306554 [cond-mat]. [Online]. Available: http : / / arxiv . org / abs / cond-mat / 0306554 \% 20http://dx.doi.org/10.1103/PhysRevLett.91.044301.

[56] M. Žnidarič and M. Horvat, "Transport in a disordered tight-binding chain with dephasing," European Physical Journal B, vol. 86, no. 2, 2013, ISSN: 14346028. DOI: $10.1140 /$ epjb/e2012-30730-9. arXiv: 1207.5952.

[57] G. Landi. (2020). Qulib - Simple yet useful functions for quantum information tasks in Mathematica, [Online]. Available: http: / / www. fmt.if.usp.br/ $\sim$ gtlandi/qulib.html. 